\title{
ASSESSMENT OF MICRONUTRIENT STATUS IN PREGNANT MALAWIAN WOMEN BEFORE AND AFTER TREATMENT FOR MODERATE MALNUTRITION
}

\author{
A Thesis \\ presented to \\ the Faculty of California Polytechnic State University, \\ San Luis Obispo
}

\author{
In Partial Fulfillment \\ of the Requirements for the Degree
}

Master of Science in Agriculture, Specialization in Food Science and Nutrition

by

Cambria M. Glosz

June 2016 
(C) 2016

Cambria M. Glosz

ALL RIGHTS RESERVED 


\section{COMMITTEE MEMBERSHIP}

TITLE:

AUTHOR:

DATE SUBMITTED:

COMMITTEE CHAIR:

COMMITTEE MEMBER:

Scott Reaves, Ph.D.

Professor of Nutrition

COMMITTEE MEMBER: $\quad$ Mary Pedersen, Ph.D.

Senior Vice Provost, Academic Programs and Planning
Assessment of Micronutrient Status in Pregnant Malawian Women Before and After Treatment for Moderate Malnutrition

Cambria M. Glosz

June 2016

Peggy Papathakis, Ph.D., RD

Professor of Nutrition 


\begin{abstract}
Assessment of Micronutrient Status in Pregnant Malawian Women Before and After Treatment for Moderate Malnutrition
\end{abstract}

\title{
Cambria M. Glosz
}

Background: Multiple micronutrient deficiencies are prevalent in pregnant women in developing countries and can result in adverse effects to both the mother and infant. Multiple micronutrient supplements or supplementary foods may be a way to combat micronutrient deficiencies.

Objective: To assess change in micronutrient and protein levels in moderately malnourished pregnant Malawian women after receiving one of three nutritional interventions.

Methods: Serum retinol, 25-hydroxyvitamin D, ferritin, vitamin $\mathrm{B}_{12}$, folate, zinc, albumin and C-reactive protein concentrations were measured in pregnant women with MUAC $>20.6 \mathrm{~cm}$ and $<23.0 \mathrm{~cm}$ at baseline $(\mathrm{n}=343)$ and after 10 weeks $(\mathrm{n}=229)$ of receiving either: 1) macro- and micronutrient fortified, peanut-based, ready-to-use supplementary food (RUSF), 2) corn-soy blended flour with a prenatal multiple micronutrient supplement (CSB-UNIMMAP), or 3) corn-soy blended flour with iron and folic acid (CSB-IFA). Each provides approximately double the RDA of most micronutrients and $900 \mathrm{kcal} /$ day. Percentages of deficiency were assessed using chi-square testing for proportions. Mean changes in concentrations were found using repeated measures MANOVA or ANOVA. Variables were transformed logarithmically if they were not on 
the normal distribution. Covariates included inflammatory status (measured by CRP), gestational age at baseline, HIV status, parity, fasting status, and MUAC at baseline. Results: Baseline micronutrient concentrations indicated high rates of deficiency in zinc (29-39\%) and albumin (37-46\%), and marginal status of retinol (26-37\%) and vitamin D (31-32\%). Adjusted mean changes in vitamin $\mathrm{B}_{12}$ concentrations from week 0 to week 10 were -17.1, -36.1, and -52.9 pg/mL for RUSF, CSB-UNIMMAP, and CSB-IFA, respectively $(\mathrm{p}=0.007)$. Adjusted mean changes in vitamin $\mathrm{D}$ concentrations from week 0 to week 10 were $6.1,3.1$, and $1.7 \mathrm{ng} / \mathrm{mL}$ for RUSF, CSB-UNIMMAP, and CSB-IFA, respectively $(\mathrm{p}=0.007)$. Adjusted mean changes in folate concentrations from week 0 to week 10 were $2.2,1.7$, and $4.0 \mathrm{ng} / \mathrm{mL}$ for RUSF, CSB-UNIMMAP, and CSB-IFA, respectively ( $\mathrm{p}=0.37$ for effect of treatment; $\mathrm{p}=0.06$ for the interaction effect of time*treatment). Changes in ferritin, zinc, albumin, retinol, and CRP were not significantly different between treatment groups.

Conclusions: Deficiencies in zinc and albumin, and marginal status of vitamin D and retinol, are common among this population of moderately malnourished pregnant Malawian women. Significant changes in vitamin D and vitamin $\mathrm{B}_{12}$ were observed from week 0 to week 10, with the RUSF group having the greatest improvements compared to the CSB-UNIMMAP and CSB-IFA treatments.

Keywords: Malnutrition, multiple micronutrients, pregnancy, supplementary foods 


\section{ACKNOWLEDGMENTS}

Thank you to my graduate advisor, Dr. Peggy Papathakis, for her extensive knowledge, guidance, and encouragement throughout this process. Thank you to my graduate committee chairs, Dr. Scott Reaves and Dr. Mary Pedersen, for their support and helpful ideas. Thank you to Tristan, Mom, and Dad for continually encouraging and standing by me throughout all of my schooling. Thank you to the funders of this study: USAID (FHI 360), USAID Feed the Future (Peanut and Mycotoxin Innovation Lab), Cal Poly San Luis Obispo Agricultural Research Initiative, U.S. Dairy Export Council, Dairy Research Initiative, Sackler Institute, and Hickey Family Foundation. Thank you to all the Mamachiponde volunteers who helped out so much on this study. And lastly, thank you to all the wonderful Malawians involved in this study: the nurses, the drivers, the community health workers, and of course, the women enrolled in this study- you will always inspire me. 


\section{TABLE OF CONTENTS}

Page

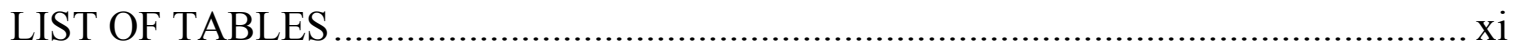

LIST OF FIGURES ......................................................................................

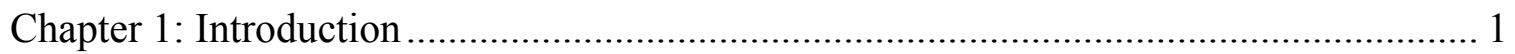

Chapter 2: Review of Literature ….................................................................... 3

2.1 Maternal Health in Malawi ................................................................................ 5

2.1.1. Diagnosis of maternal malnutrition and body composition....................... 6

2.1.2 Malnutrition and impact on maternal and infant health ............................ 9

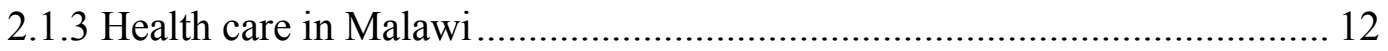

2.2. Micronutrient deficiency in pregnancy ................................................... 13

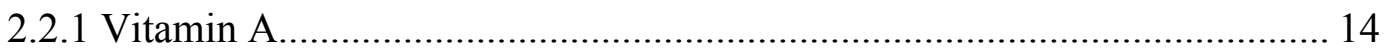

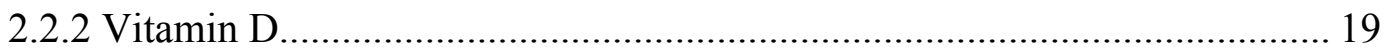

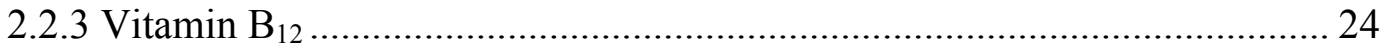

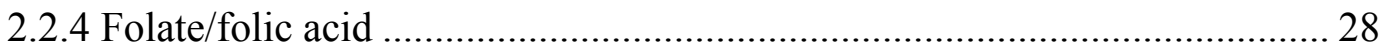

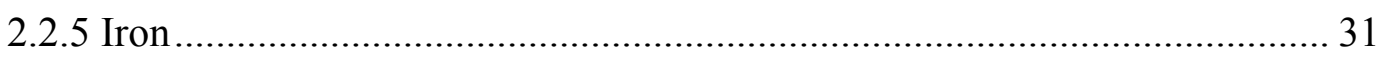

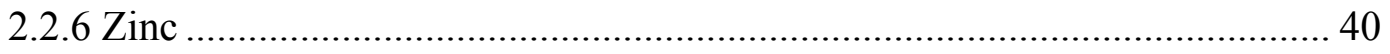

2.3 Nutrition, infections, and inflammation ...................................................... 47

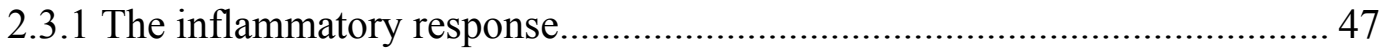

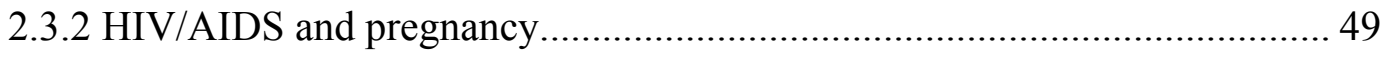


2.3.3 Inflammation and anemia of chronic disease.

2.4 Multiple micronutrient supplementation interventions....................................... 54

2.4.1 Maternal outcomes of multiple micronutrient supplementation.................. 55

2.4.2 Infant outcomes of maternal multiple micronutrient supplementation........ 59

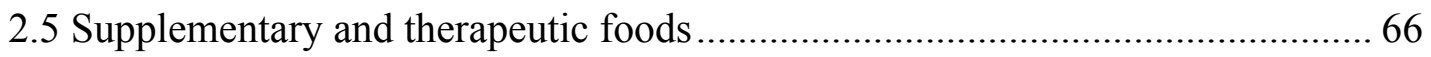

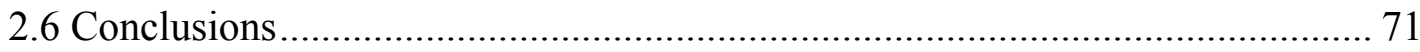

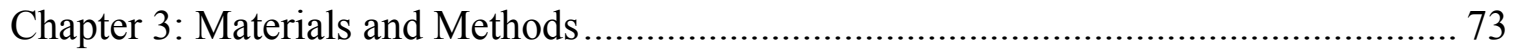

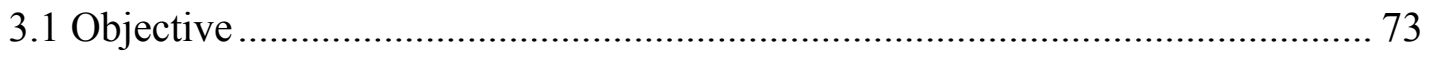

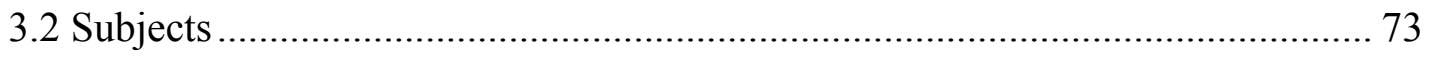

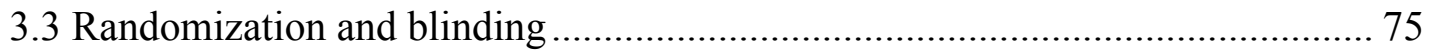

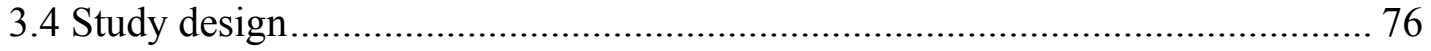

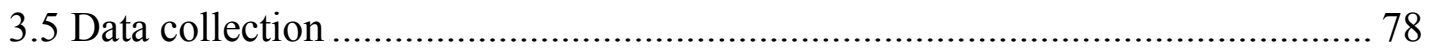

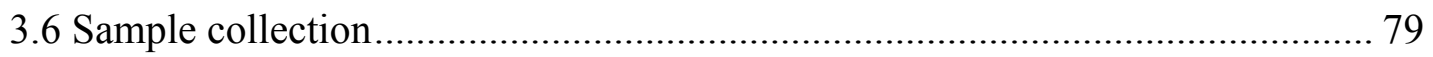

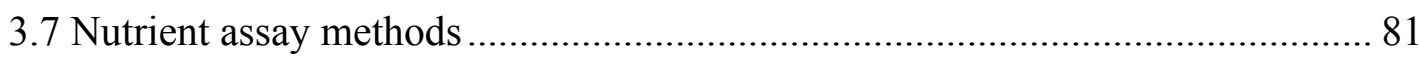

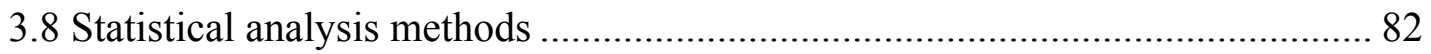

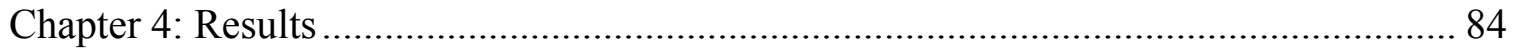

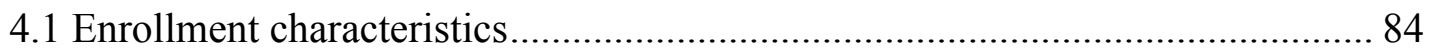

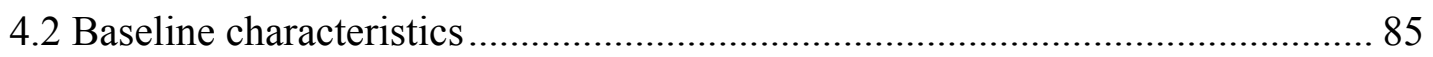

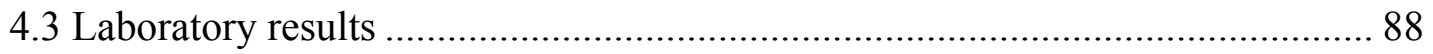

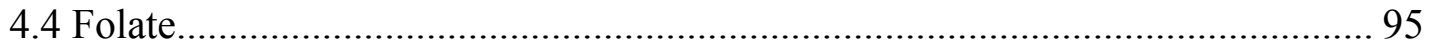




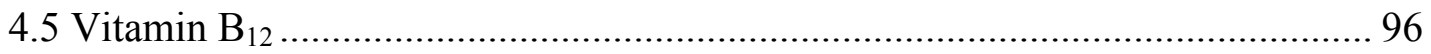

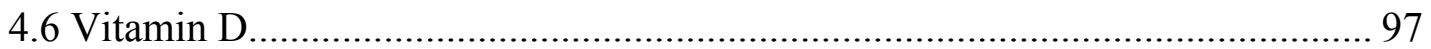

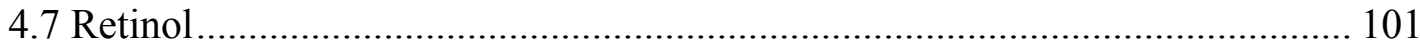

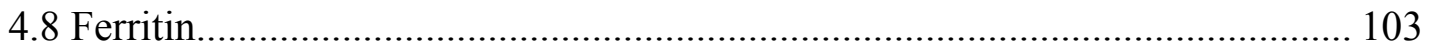

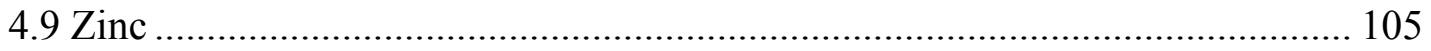

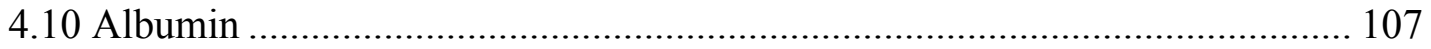

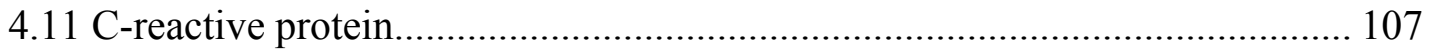

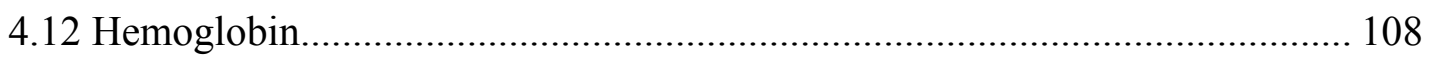

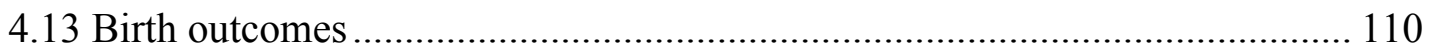

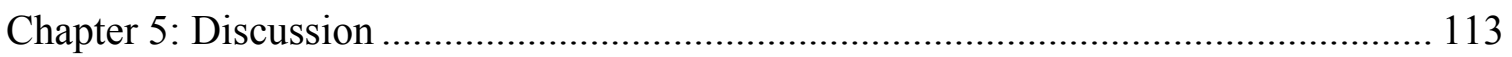

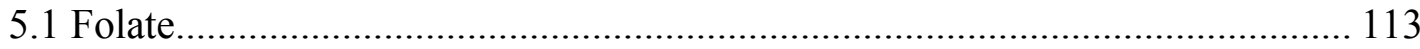

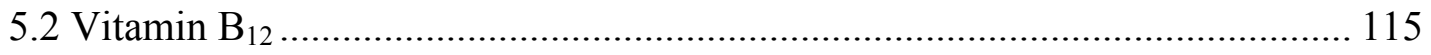

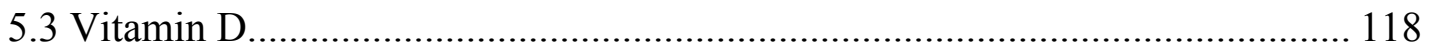

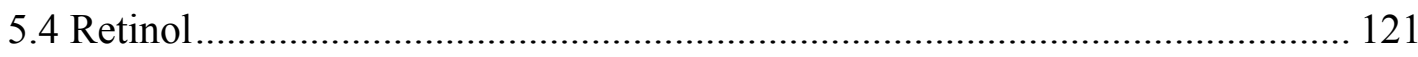

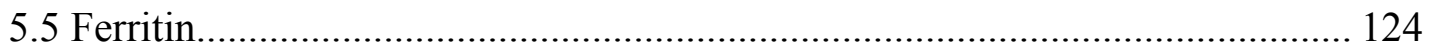

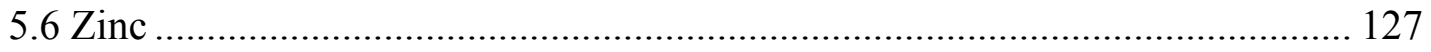

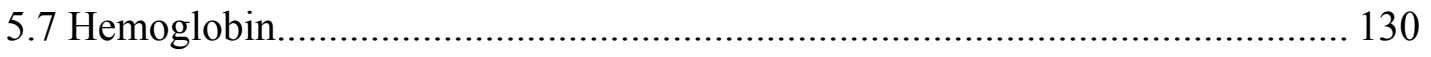

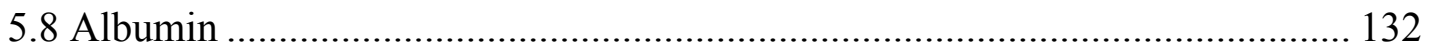

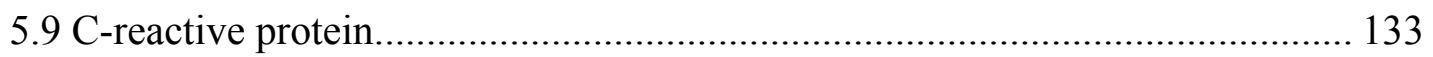

5.10 Birth and infant measurements .................................................................. 135 
5.11 Strengths and limitations.

Chapter 6: Conclusions

140

References.

142

\section{APPENDICES}

Appendix A: Recommended Dietary Allowances (RDA) or Adequate Intakes (AI)

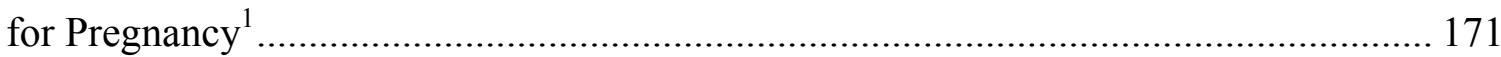

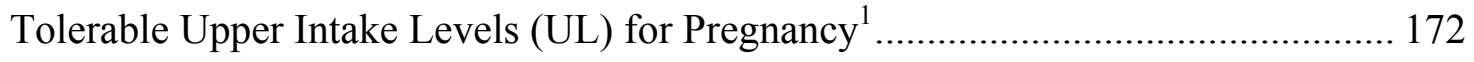

Appendix B: Nutrient Comparison by Treatment Group ...................................... 173

Appendix C: Micronutrient Sub-study Form .......................................................... 174

Appendix D: Micronutrient Sub-study Procedures................................................... 175

Appendix E: Mamaponde Micronutrient Blood Draw Informed Consent .................... 178 


\section{LIST OF TABLES}

Table

Page

Table 2.1. Maternal micronutrient deficiencies and their potential impact on fetal and

infant neurodevelopment. (Adapted from Kerac et al., 2014) 12

Table 2.2. Effects of supplementing HIV-infected pregnant and lactating women on the mean serum concentrations of retinol in their infants at 6 weeks and 6 months of

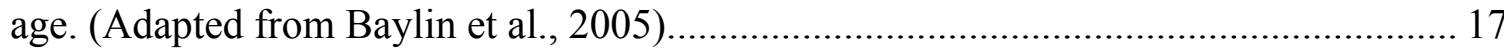

Table 2.3. Serum 25(OH)D concentrations and deficiency cut-off values...................... 21

Table 2.4. Dietary factors that can inhibit or enhance absorption of iron (FAO \& WHO, 1998; S. S. Gropper et al., 2009).

Table 2.5. Iron requirements and losses during a healthy pregnancy and delivery.

Adapted from (FAO \& WHO, 1998).

Table 2.6 Proposed lower cut-offs for assessment of zinc deficiency during

pregnancy.....

Table 2.7. Infant outcomes from trials of maternal multiple micronutrient

supplementation. (Fawzi et al., 2007; A. H. Shankar et al., 2008; West et al., 2014;

Zeng et al., 2008)

Table 3.1 Assays used for analysis of nutrients and proteins

Table 3.2 Normal deficiency and marginal status cut-off values for pregnancy and deficiency values in inflammatory state 81

Table 4.1 Baseline characteristics of the participants at enrollment, by treatment

group 86 
Table 4.2. Unadjusted serum nutrient/protein means and adjusted proportions of

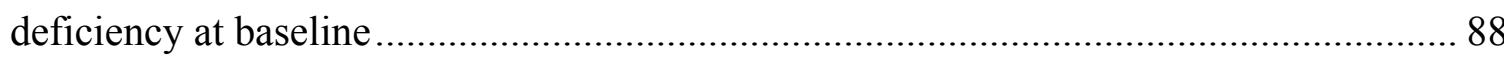

Table 4.3 Baseline adjusted mean (SE) concentrations by treatment group ${ }^{1}$................. 89 Table 4.4 Adjusted least square mean concentrations (SE) at week 10 by treatment

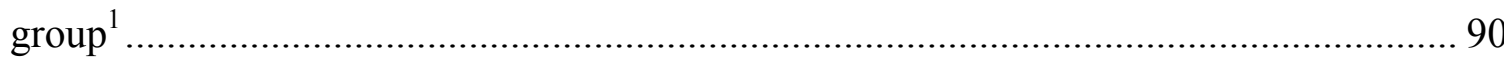

Table 4.5 Mean change in adjusted concentrations from week 0 to week 10, by

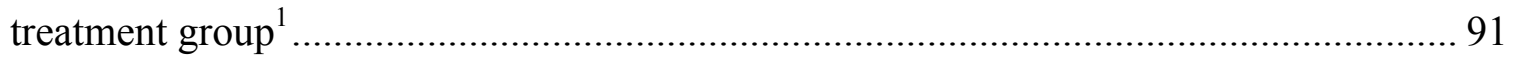

Table 4.6 Percentage of deficiency or elevation by treatment at week 0 and week $10 \ldots 92$ Table 4.7 Adjusted means (least square) by treatment and two-way Tukey comparison

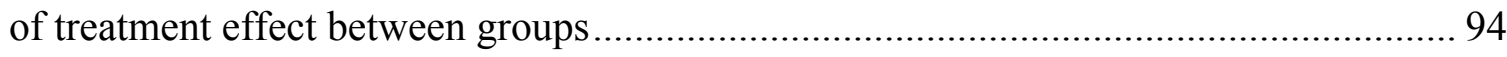

Table 4.8 Difference in adjusted mean vitamin D concentrations $(\mathrm{ng} / \mathrm{mL})$ with and without the interaction of time*treatment added into the model 98

Table 4.9 Comparison of vitamin D concentrations $(\mathrm{ng} / \mathrm{mL})$ at week 10 by clinic site and clinic district

Table 4.10 Proportions of sufficient, marginal, and deficient retinol status by treatment group, from week 0 to week 10, controlling for inflammation 102

Table 4.11 Unadjusted mean \pm SD (n) of birth and 3-month measurements by treatment group 110 


\section{LIST OF FIGURES}

Figure

Page

Figure 2.1. The effects of maternal malnutrition, with potential causes and

consequences throughout the life cycle. (Excerpted from Ahmed et al., 2012.) 4

Figure 2.2. Malawi, a country in southeastern Africa. Excerpted from

(http://commons.wikimedia.org) 5

Figure 2.3. Factors involved in maternal malnutrition and effects on maternal and infant health. (Adapted from Lartey, 2008).

Figure 2.4. Values of arm muscle area throughout the pregnancies of HIV-infected Malawian women who were either fully exposed (all of the last 30.5 days) to famine season or not exposed (zero of the last 30.5 days). (Excerpted from Thomas et al., 2013) 8

Figure 2.5. The dietary supply of zinc and the risk of zinc deficiency in African countries. Excerpted from (Joy et al., 2014).

Figure 2.6. Maternal serum zinc concentration and newborn NTD incidence

$(0=$ controls; $1=$ cases with NTDs). Excerpted from (Dey et al., 2010) 44

Figure 2.7. The decline of average serum zinc concentrations throughout gestation in previous literature, iron group, and iron-zinc group. Excerpted from (L. E. Caulfield et al., 1999). 46

Figure 2.8. The relationships between human immunodeficiency virus (HIV), immunity, and malnutrition. Excerpted from (Macallan, 1999a). 50

Figure 4.1 Flow chart of enrollment into the study 84 
Figure 4.2 Change in folate means from week 0 to week 10 by treatment group.

Figure 4.3 Change in adjusted mean vitamin $\mathrm{B}_{12}$ concentrations $(\mathrm{pg} / \mathrm{mL})$ by treatment

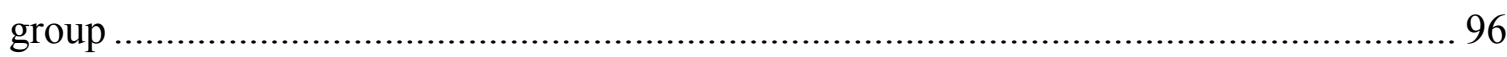

Figure 4.4 Correlation of week 0 to week 10 concentrations of vitamin $B_{12}(\mathrm{pg} / \mathrm{mL})$

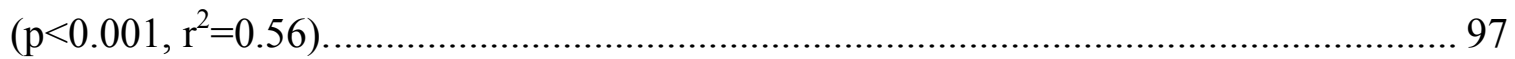

Figure 4.5 Mean increase in vitamin D concentrations from week 0 to week 10, by

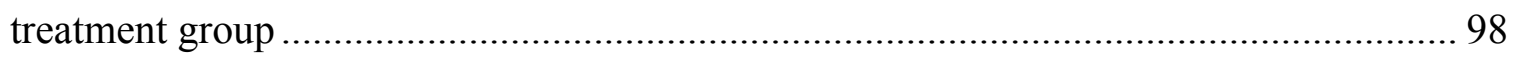

Figure 4.6 Correlation of baseline maternal MUAC and vitamin D concentration at

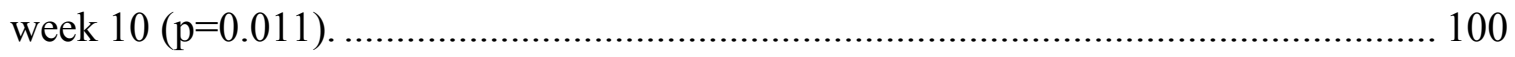

Figure 4.7 Percent of deficient, marginal, and sufficient retinol status by treatment

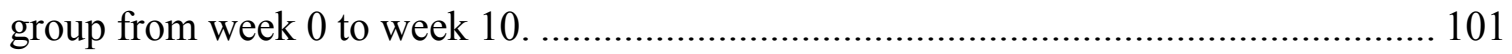

Figure 4.8 Correlation of baseline retinol $(\mu \mathrm{mol} / \mathrm{L})$ and baseline CRP $(\mathrm{mg} / \mathrm{L})$

$(\mathrm{p}<0.0001)$

Figure 4.9 Correlation of parity and baseline ferritin concentrations (ng/mL)

$(\mathrm{p}<0.0001)$ 104

Figure 4.10 Correlation of week 10 ferritin $(\mathrm{ng} / \mathrm{mL})$ and week $10 \mathrm{CRP}(\mathrm{mg} / \mathrm{L})$

$(\mathrm{p}<0.0001)$

Figure 4.11 Adjusted serum zinc concentrations at baseline and week 10 by treatment

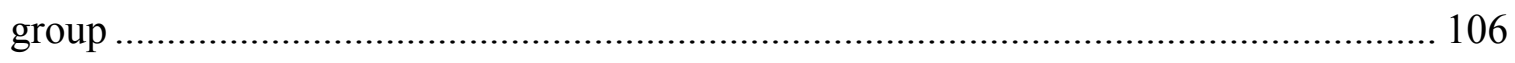

Figure 4.12 Correlation of baseline hemoglobin $(\mathrm{g} / \mathrm{dL})$ to baseline ferritin $(\mathrm{ng} / \mathrm{mL}) \ldots . .109$ 


\section{CHAPTER 1: INTRODUCTION}

Malnutrition is a global problem, especially in developing countries, with pregnant women being particularly vulnerable to becoming undernourished. Pregnancy greatly increases requirements for many nutrients (Lartey, 2008). An environment of poverty and food shortages results in increased prevalence of maternal malnutrition, which can cause short-term and long-term health consequences to both a mother and her child. Poor birth outcomes such as low birth weight (LBW) infants, stillbirths, preterm delivery, and neonatal mortality are common in undernourished mothers (Ahmed, Hossain, \& Sanin, 2012; Lartey, 2008).

Infectious diseases, such as HIV and malaria, are common in African women and can further worsen nutritional status (Lartey, 2008). Malawi is a low-income country in sub-Saharan Africa that has one of the highest rates of maternal mortality worldwide, at 634 deaths per 100,000 live births (The World Bank, 2015). Similar to other resourcelimited countries, Malawian women are afflicted with a combination of chronic lack of energy intake, insufficient weight gain during pregnancy, anemia, and micronutrient deficiencies (Ahmed et al., 2012).

Malnourished women have a higher rate of mortality during pregnancy and childbirth than women who are properly nourished (Ahmed et al., 2012). Micronutrient deficiencies are common in those who are malnourished, and even moderate deficiencies can have severe negative effects on the mother and fetus. Children who are born in an undernourished state are at increased risk of having stunting and malnutrition later in life. Malnutrition can become an intergenerational vicious cycle that is difficult to recover from (Ahmed et al., 2012). 
The most common micronutrient deficiencies worldwide are iron, vitamin A, iodine, and zinc (Ahmed et al., 2012). Deficiencies in these nutrients during pregnancy, in addition to vitamin $\mathrm{D}$, vitamin $\mathrm{B}_{12}$, and folate, can lead to a myriad of negative maternal consequences, including night blindness, increased susceptibility to infections, anemia, and preeclampsia; infant-related consequences include small for gestational age (SGA) and LBW newborns, increased risk of premature births and stillbirths, neural tube defects, and decreased fetal cognitive development (Ahmed et al., 2012; Christian, Schulze, Stoltzfus, \& Jr, 1998; Kennedy, Nantel, \& Shetty, 2003).

The current micronutrient supplementation standard of care for pregnant women in most developing countries is supplementation with iron and folic acid. Recent research has questioned whether multiple micronutrients would be more beneficial to maternal and infant nutrition than iron and folic acid alone (Bhutta, Imdad, Ramakrishnan, \& Martorell, 2012). It is crucial to investigate the prevalence of micronutrient deficiencies in pregnant Malawian women and to determine if supplementation with multiple micronutrients or ready-to-use fortified foods during pregnancy can improve nutritional status greater than iron and folic acid supplements. In order to better target nutritional treatment, determination of micronutrient status and prevalence of deficiencies is helpful. In addition, monitoring change in serum micronutrient levels during treatment will help to determine which treatment is most effective in delivering micronutrients. 


\section{CHAPTER 2: REVIEW OF LITERATURE}

Maternal malnutrition is a global burden that is related to millions of deaths of women and children each year (Ahmed et al., 2012). Maternal nutrition refers to the nutritional needs of a woman before pregnancy, throughout pregnancy, and during lactation. Pregnant women who are undernourished prior to conception are more likely to worsen their nutritional status due to the increased energy and nutrient demands from the growing fetus (Ahmed et al., 2012). Women in developing countries tend to perform hard physical labor throughout the entire length of their pregnancies, which further increases their nutrient requirements (Lartey, 2008). The additional nutrient demands during pregnancy and lactation can cause the woman's tissues to become depleted, making this a high-risk period. In developing countries, it is common for women to have frequent pregnancies due to a lack of birth control (Ahmed et al., 2012). Closely spaced pregnancies result in insufficient time to replenish nutrient and energy stores before the next pregnancy (Lartey, 2008).

Women who are malnourished during pregnancy have a higher risk of mortality than healthy pregnant women (Lartey, 2008). Maternal malnutrition becomes a vicious cycle that gets handed down to subsequent generations. A malnourished infant will often grow up to be a malnourished adult (Ahmed et al., 2012; Fig. 2.1). 


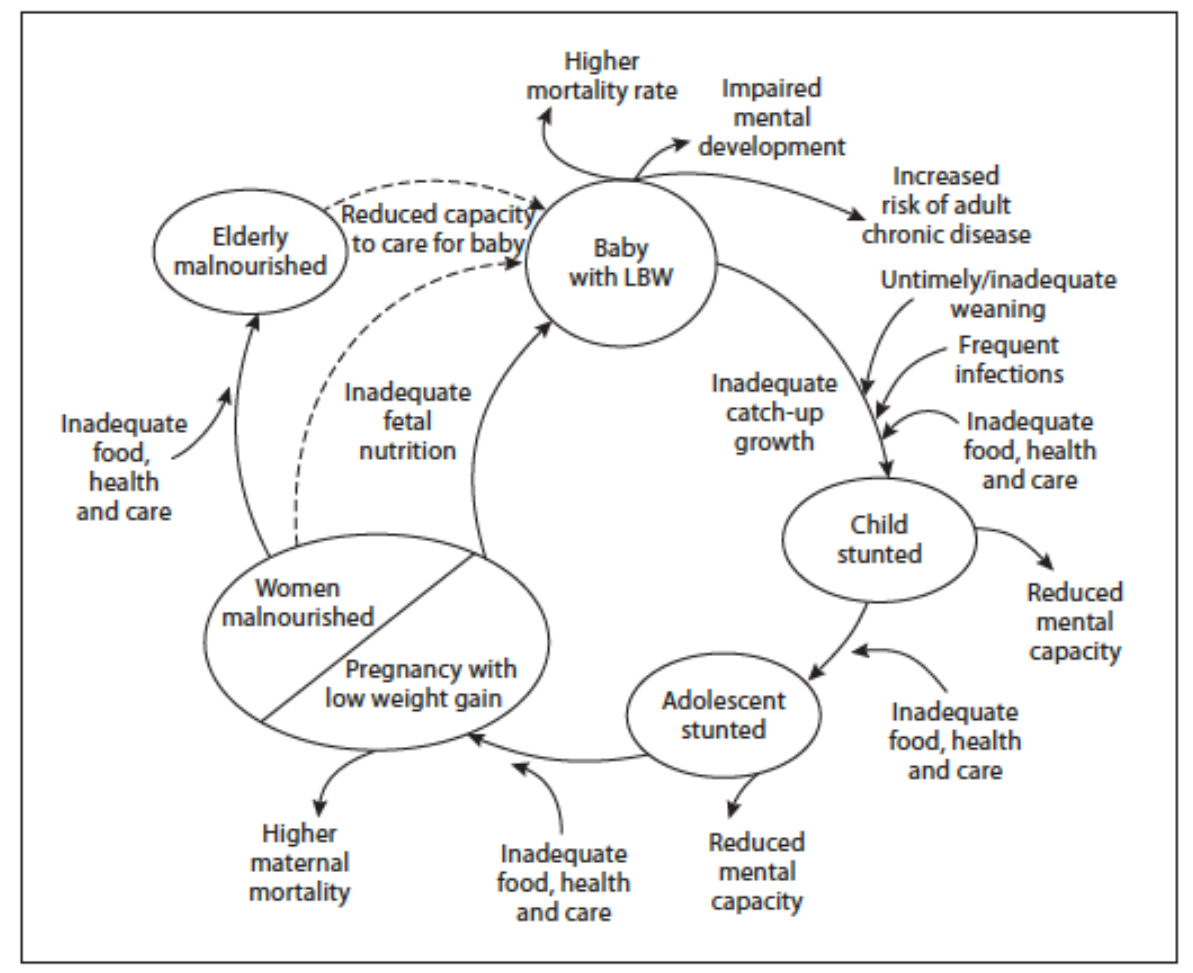

Figure 2.1. The effects of maternal malnutrition, with potential causes and consequences throughout the life cycle. (Excerpted from Ahmed et al., 2012.)

Malnutrition in the form of micronutrient deficiency is most common among young children and women of reproductive age. Even moderate deficiencies during pregnancy can have serious adverse effects for the mother and fetus (Ahmed et al., 2012). Micronutrient deficiencies are sometimes referred to as "hidden hunger", because the effects on the body can go unnoticed and are not as visual as protein-energy malnutrition, especially when the deficiency is subclinical (Kennedy et al., 2003). Almost one-third of the world is affected by a deficiency in one or more micronutrient (Ahmed et al., 2012). Improving the micronutrient status of pregnant women is one proposed method to combat maternal malnutrition and improve birth outcomes (Black, 2001). 


\subsection{Maternal Health in Malawi}

Malawi is a resource-limited, landlocked country in southeastern Africa with a population of 16.36 million (The World Bank, 2015) (Figure 2.2). One out of every 36 pregnant women will die during pregnancy or childbirth in Malawi, one of the highest rates in the world. The main causes of maternal mortality include hemorrhage, sepsis, hypertension, and other pregnancy complications, but the indirect causes include nutritional deficiencies and malaria (UNICEF, 2013).

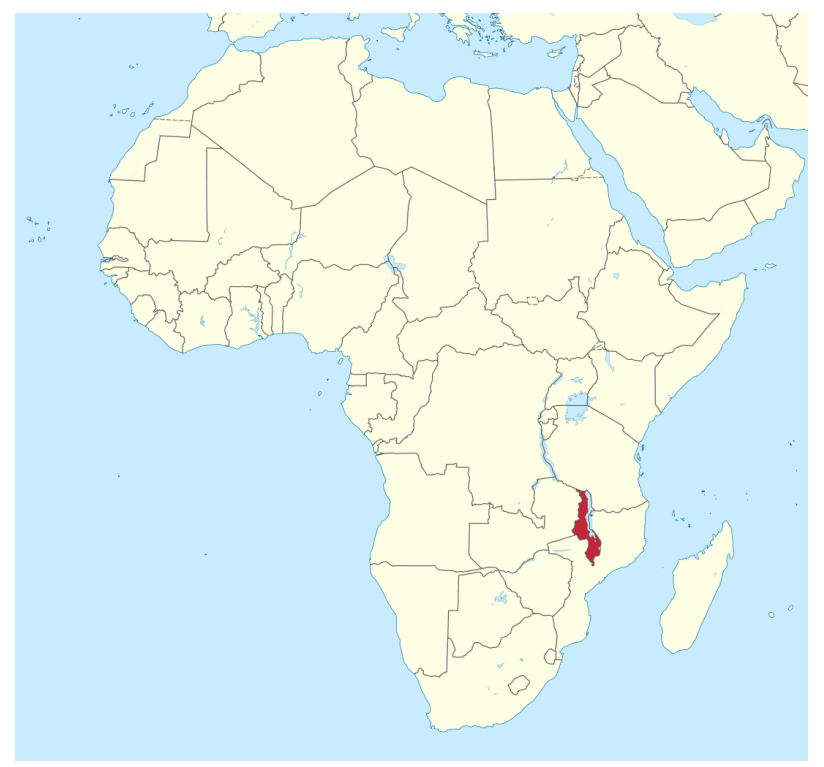

Figure 2.2. Malawi, a country in southeastern Africa. Excerpted from (http://commons.wikimedia.org).

Another factor that further compromises maternal nutrition is infection with HIV, which disproportionately affects Malawian women. The average prevalence of HIV in Malawian adults is 12\% (UNICEF, 2013); approximately $58 \%$ of the individuals with HIV are female (UNAIDS, 2012). High fertility rates in Malawi increase a woman's risk for malnutrition, due to the inability to recover nutritionally from the previous pregnancy. The total fertility rate in Malawi in 2014 was 5.26 children born per woman (Central 
Intelligence Agency, 2014). Figure 2.3 summarizes the factors involved with maternal malnutrition and the consequences it can have on both maternal and infant health.

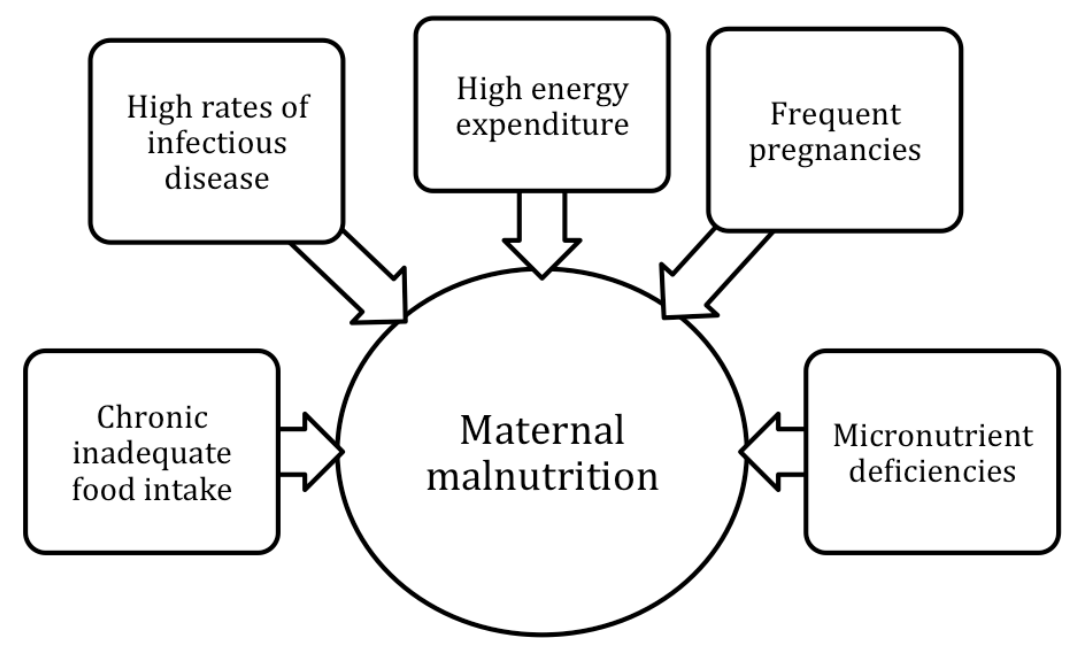

Figure 2.3. Factors involved in maternal malnutrition and effects on maternal and infant health. (Adapted from Lartey, 2008).

Women of reproductive age in Malawi are at high risk of contracting infectious diseases, including HIV/AIDS, tuberculosis (TB), pneumonia, malaria, and parasitic infections. Some of these diseases and their relation to maternal malnutrition will be discussed in detail in later sections.

\subsubsection{Diagnosis of maternal malnutrition and body composition}

There is no international agreement on the diagnostic criteria to identify pregnant women as malnourished and when to enroll them in nutritional intervention programs. Mid-upper arm circumference (MUAC) is commonly used to classify malnutrition in all populations. MUAC changes very little throughout pregnancy and is not dependent on gestational age, unlike weight (Ververs, Antierens, Sackl, Staderini, \& Captier, 2013; WHO, 1995). There is some inconsistency with the MUAC cut-off guidelines for malnutrition in pregnant women. MUAC values below 22 and $23 \mathrm{~cm}$ are associated with 
moderate malnutrition and adverse effects during pregnancy, including LBW (Karim \& Mascie-Taylor, 1997), IUGR, and premature infants (Verhoeff et al., 2001; Ververs et al., 2013). MUAC values below $20.7 \mathrm{~cm}$ during pregnancy are associated with severe maternal malnutrition and fetal growth retardation (The Sphere Project, 2011). Guidelines are not yet universal in recommending specific cut-off values for enrolling pregnant women into nutritional feeding programs (Ververs et al., 2013).

There are other measurements of body composition that can be used to determine nutritional status. A healthy pregnant woman's arm fat area (AFA) rises in the first trimester and decreases in the third trimester because fat stores are being supplied to the fetus. Arm muscle area (AMA) of a healthy pregnant woman rises in the third trimester and should stay elevated until at least 8 weeks after delivery (Taggart, Holliday, Billewicz, Hytten, \& Thomson, 1967; R. M. Thomas et al., 2013).

Pregnant women living in resource-limited countries tend to have different patterns of body composition compared to pregnant women in developed countries, due to a combination of poor dietary intake, frequent pregnancies, high amounts of physical labor during pregnancy, and acute infections (R. M. Thomas et al., 2013). The rainy season in many African countries, including Malawi, is associated with higher rates of food shortages, physical labor, and malarial infections, which can lead to increased maternal morbidity and reduced weight gain during pregnancy (Branca, Pastore, Demissie, \& Ferro-Luzzi, 1993; Friis et al., 2004b; R. M. Thomas et al., 2013).

Patterns of body composition in HIV-infected pregnant women are not as well understood. A longitudinal study of HIV-infected women in Malawi measured anthropometrics and body composition during pregnancy. Women who were pregnant 
during the famine season (from August to March) experienced significant decreases in MUAC and AMA (Figure 2.4), as well as a lower rate of gestational weight gain and increased loss of AFA, compared to pregnant women who were not exposed to the famine season (R. M. Thomas et al., 2013).

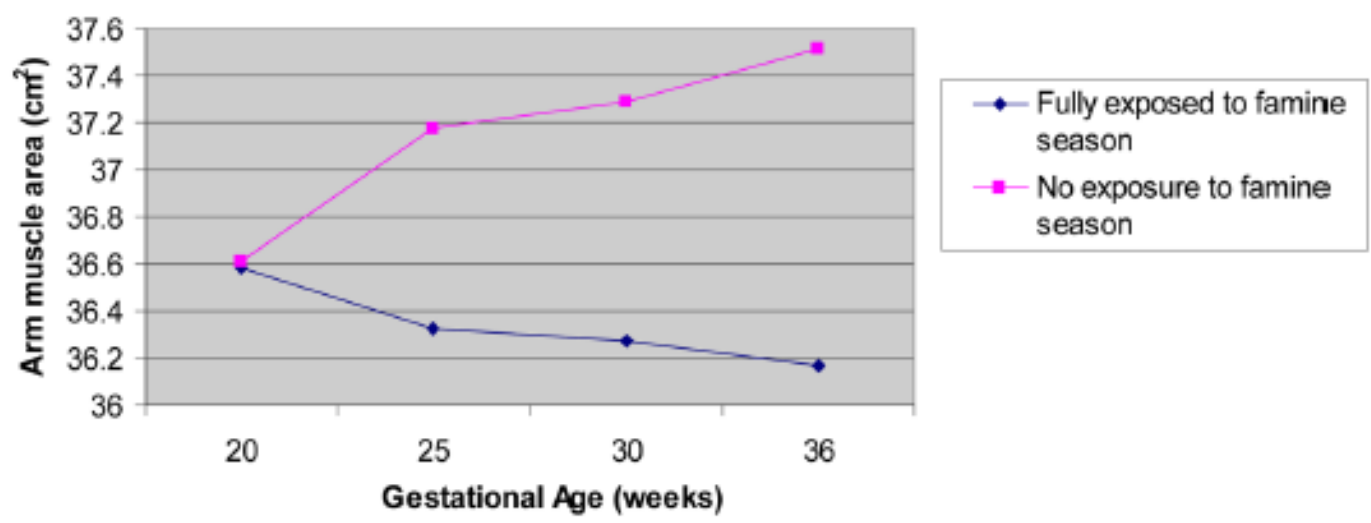

Figure 2.4. Values of arm muscle area throughout the pregnancies of HIV-infected Malawian women who were either fully exposed (all of the last 30.5 days) to famine season or not exposed (zero of the last 30.5 days). (Excerpted from Thomas et al., 2013)

The increase of AMA during the third trimester in women not exposed to the famine season in the study by Thomas et al. (2013) is similar to the normal pattern seen in healthy pregnant women. However, the significant loss of MUAC and AMA in women exposed to the famine season is consistent with a study of Tanzanian HIV-infected pregnant women, who lost an average of $1 \mathrm{~cm}$ from their MUAC during the second and third trimesters (R. M. Thomas et al., 2013; Villamor, Saathoff, Msamanga, et al., 2005). Loss of AMA in HIV-infected pregnant women has been linked to increased risk of infection, progression of HIV, death, and possible increase in risk of mother-to-child transmission of HIV (Macallan, 1999b; Villamor, Saathoff, Msamanga, et al., 2005).

A study in HIV-infected Malawian pregnant women analyzed dietary patterns and anthropometrics (Ramlal et al., 2015). In this population, the mean energy intake was 
1,378 calories per day. Based on 24-hour dietary recalls, women were placed into one of three diet clusters: animal-based, plant-based, or grain-based. Women consuming a grainbased diet had the lowest wealth index and were most exposed to the famine season, resulting in lower intake of calories, protein, fat and iron, compared to women consuming the animal- or plant-based diets. Women consuming grain-based diets had significantly lower AFA compared to the plant-based group, however, the grain-based group had higher AMA compared to the animal-based group. This may be because women in the grain-based group would be more likely to perform manual labor, such as farming, due to their lower wealth index. This study provided an interesting look into Malawian dietary patterns and the authors conclude that this population could benefit from increased dietary diversity and caloric and nutrient intake, especially during pregnancy (Ramlal et al., 2015).

\subsubsection{Malnutrition and impact on maternal and infant health}

Malnutrition can affect maternal health by increasing a woman's risk of anemia, micronutrient deficiencies, short stature, low BMI, and maternal mortality (Bhutta \& Das, 2014; Black et al., 2008). Possible consequences of micronutrient deficiency during pregnancy include maternal or fetal death, increased labor complications, anemia, abnormal fetal organ development, compromised fetal growth, and reduced immunity of the newborn (Black, 2001; Ramakrishnan, Manjrekar, Rivera, Gonzáles-Cossío, \& Martorell, 1999). Over twenty percent of maternal deaths can be attributed to inadequate nutrition during pregnancy (Bhutta \& Das, 2014). The World Health Organization (WHO) defines maternal mortality as the death of a woman during the pregnancy or 
within 42 days after pregnancy has ended, due to a cause related to or worsened by the pregnancy itself (WHO, 2015b).

Maternal malnutrition can also have a great impact on the health and nutritional status of her child. Low maternal BMI can contribute to intrauterine growth restriction (IUGR), LBW infants, and neonatal mortality (Arimond et al., 2013). There is a growing amount of research about the "first 1,000 days" of a child's life (from conception to age two) as a crucial time to prevent childhood stunting. During this time period, maternal and infant undernutrition can cause irreversible damage (Arimond et al., 2013; Victora et al., 2008).

Maternal anthropometrics can be predictors of infant size. A study done on Malawian HIV-infected pregnant women found that maternal MUAC, AFA and AMA strongly predicted the weight of her infant, with the strongest predictor being MUAC. In this study, every $1 \mathrm{~cm}$ increase in maternal MUAC corresponded to a $31.8 \mathrm{~g}$ increase in infant birth weight (R. Thomas et al., 2012).

Fetal and infant malnutrition can result in both immediate and long-term consequences. Proximate concerns include a higher risk of being born with LBW, small for gestational age (SGA), or IUGR, which then increases the likelihood of being stunted as a child. Mothers who have short stature or low BMI are more likely to deliver newborns that are LBW or SGA (Adair, 2014). These smaller infants are also more likely to have decreased cognitive and educational abilities, higher rates of morbidity and mortality, and impaired immunity (Adair, 2014; R. Thomas et al., 2012).

Long-term consequences of fetal undernutrition include decreased cognitive development and motor skills, as well as a higher chance of developing chronic disease 
later in life (Arimond et al., 2013; The World Bank, 2006). Most of the brain development of an infant occurs during the first 1,000 days after conception, thus, intrauterine life is critical. The neural tube of the fetus, which will form the brain and spinal cord, begins to form only 22 days after conception. The brain develops the most between 24 and 42 weeks gestation, making infants who are born preterm at higher risk of not having a fully developed neurological system (Kerac et al., 2014). The nutrients that are most crucial for neurodevelopment include iron, zinc, folate, vitamin A, selenium, iodine, and choline (Georgieff, 2007). These nutrients impact various areas of brain development, thus a deficiency in one may have differing effects on cognitive growth than another. Specific micronutrient deficiencies and their effect on fetal neurodevelopment are shown in Table 2.1. However, micronutrient deficiencies are rarely singular and it is common for pregnant women to be lacking in multiple nutrients (Kerac et al., 2014). Essential fatty acids (EFAs), especially docosahexaenoic acid (DHA), are necessary for the function of membranes and synapses, as well as myelination in the brain (Georgieff, 2007). Infants who are born preterm are at risk of EFA deficiency because the EFAs accumulate in the brain during the third trimester (Prado \& Dewey, 2014). Other long-term consequences of fetal undernutrition include increased risk of chronic disease later in life, such as cardiovascular disease. This may be due to increased stress in the womb (Roseboom, Painter, van Abeelen, Veenendaal, \& de Rooij, 2011). A study of individuals who were born during a famine in the Nigerian civil war (1967-1970) found that they had increased risk of type 2 diabetes and hypertension, compared to those not exposed in utero to the famine (Hult et al., 2010). 
Table 2.1. Maternal micronutrient deficiencies and their potential impact on fetal and infant neurodevelopment. (Adapted from Kerac et al., 2014)

\section{Nutrient Deficiency $\quad$ Fetal and Infant Neurodevelopmental Risk}

Folate Neural tube defects

Iron

Impaired language ability and fine motor skills at age 5

Vitamin A

Anemia and auditory loss

Zinc

Autonomic and cerebellar dysfunction

The perinatal period, which is defined as the period between 22 weeks gestation and 7 days postpartum, requires high nutrient and energy intake in order to support the increased growth rates of the fetus and newborn (Makrides, Anderson, Gibson, \& Collins, 2013; WHO, 2015a). Inadequate nutrient supply to the fetus can result in LBW infants, who are more likely to have lower cognitive abilities later in life, compared to infants of average birth weight. This inadequate nutrient supply could occur due to a number of factors, including poor maternal nutrition, preterm birth, or placental insufficiency leading to reduced flow of nutrients to the fetus. Preterm infants have been found to have reduced cognitive abilities compared to normal term babies (Makrides et al., 2013).

\subsubsection{Health care in Malawi}

Antenatal care (ANC) in Malawi is found in both rural and urban centers. From 2008-2012, 94.7\% of pregnant women attended at least one visit to ANC. However, only $45.5 \%$ of these women attended ANC at least four times (UNICEF, 2013). Many women don't attend ANC until their second or third trimester. Seventy-three percent of women deliver in an institutional setting, such as a clinic or hospital (UNICEF, 2013).

Supplementary feeding programs (SFPs) are common for severely or moderately malnourished children and lactating mothers, but SFPs are not currently widespread for 
pregnant women. Records from 2008 indicate that 210 ANC centers in 28 districts of Malawi provided SFPs for 6,500 pregnant and lactating mothers. The current standard of care is iron and folic acid supplements for all pregnant women. Malnourished pregnant and lactating women receive a corn-soy flour blend (CSB) ration of 5 kilograms per fortnight at some, but not all, ANC clinics (World Food Programme, 2008b).

Malaria is a major health issue affecting Malawi, with especially devastating effects for pregnant women and children. In 2012, malaria accounted for 63 out of 100,000 deaths in the Malawian population, while accounting for 1 out 12 deaths in children under five (President's Malaria Initiative, 2015). Malaria in pregnancy can lead to perinatal mortality, LBW infants and premature delivery (Schantz-Dunn \& Nour, 2009). Intermittent preventive treatment in pregnancy (IPTp) gives doses of the antimalarial drug sulphadoxine-pyrimethamine (SP) to prevent maternal malarial infection. In Malawi, the first IPTp-SP dose is given as early as possible in the second trimester, with the second dose administered at least one month after the first dose (WHO, 2012b). In 2012, 53\% of women received two or more doses of IPTp-SP during their previous pregnancy (President's Malaria Initiative, 2015). SP is contraindicated in women receiving cotrimoxazole prophylaxis (for prevention of mother-to-child-transmission of HIV). Pregnant women also receive insecticide treatment bed nets during pregnancy to further reduce risk of contracting malaria (WHO, 2012b).

\subsection{Micronutrient deficiency in pregnancy}

Vitamins and minerals, collectively called micronutrients, are essential for the proper growth of a fetus. Inadequate intake of micronutrients during pregnancy can result in problems for both the mother and fetus. Common deficiencies among pregnant women 
worldwide are vitamins $\mathrm{A}, \mathrm{B}_{6}, \mathrm{~B}_{12}, \mathrm{C}, \mathrm{D}, \mathrm{E}$, and folate and minerals iron, iodine, and zinc (Black et al., 2008; Kawai, Spiegelman, Shankar, \& Fawzi, 2011).

There are various confounding factors that can make it difficult to determine the prevalence and magnitude of maternal micronutrient deficiencies, especially in developing countries. One is that micronutrient deficiencies tend to coexist, which can make it difficult to determine which deficiency is causing which outcome. There is also intra-individual variability in bioavailability of nutrients, which can lead to different nutrient requirements for different individuals (Black, 2001). The micronutrients that will be discussed in detail in this review are vitamins $\mathrm{A}, \mathrm{B}_{12}, \mathrm{D}$, and folate, and minerals zinc and iron.

\subsubsection{Vitamin A}

Vitamin A, a fat-soluble nutrient, is necessary for visual function, immunity, cell growth, integrity of epithelial cells, lung development, production of red blood cells, and reproduction (Checkley et al., 2010; WHO, 2009). Retinol and its oxidation products are necessary for the transcription of hundreds of genes, especially within the visual and reproductive systems (S. S. Gropper, Smith, \& Groff, 2009). A diet that is deficient in vitamin A will result in the inability to adequately support vision, immunity, and cellular processes. Some periods of the lifecycle require increased amounts of vitamin A to support these systems, including pregnancy, lactation, infancy and childhood (WHO, 2009).

Serum retinol is a measure of the body's reserves of vitamin A and it is controlled by homeostasis in the body. Serum retinol levels do not decrease until the stores in the body are compromised. Retinol levels are impacted by infection, protein status, and 
levels of other micronutrients (dePee \& Dary, 2002). The cut-off value for using retinol to determine vitamin A deficiency (VAD) is generally $<0.70 \mu \mathrm{mol} / \mathrm{L}$. However, some sources indicate that for pregnant and lactating women, a cut-off value of $<1.05 \mu \mathrm{mol} / \mathrm{L}$ should be used to indicate marginal vitamin A status, with $<0.70 \mu \mathrm{mol} / \mathrm{L}$ representing vitamin A deficiency. Severe VAD is characterized by retinol levels $<0.35 \mu \mathrm{mol} / \mathrm{L}$ (Sommer \& Davidson, 2002; WHO, 2009). Some research has indicated the use of different cut-off values to account for inflammation. Thurnham \& McCabe (2012) suggest using $<0.60 \mu \mathrm{mol} / \mathrm{L}$ to classify VAD with inflammation. See Appendix A for the recommended dietary allowances (RDA) and tolerable upper intake levels (UL) during pregnancy for vitamin A and all other micronutrients discussed in this review. There is a risk of excessive intake of vitamin A during pregnancy, which can cause fetal toxicity and birth defects. Women who are pregnant or may become pregnant should not exceed 3,000 $\mu$ g retinol equivalents (RE) per day (FAO \& WHO, 1998).

A WHO analysis of vitamin A concentrations from 1995-2005 found that over 19 million pregnant women worldwide are estimated to have low serum retinol, which represents $15.3 \%$ of pregnant women living in countries at highest risk for VAD. This study estimated that $13.7 \%$ of Malawian pregnant women were deficient in vitamin A, which categorizes Malawi as having a moderate public health problem in terms of VAD (WHO, 2009).

VAD in pregnant women is associated with severe anemia, wasting, and morbidity from infectious diseases. VAD and infections have a complex and bidirectional relationship, in that each influences and increases the risk of the other (Christian et al., 1998). Infections can increase the risk of VAD, and individuals (especially young 
children and pregnant women) with VAD are more susceptible to developing infectious diseases (Christian et al., 1998). Vitamin A is involved in the immune system via retinoic acid, which stimulates the activity of phagocytes and cytokines, and preserves concentrations of natural killer cells. Individuals with VAD are less able to fight infections because VAD causes reduced regeneration of mucosal epithelial cells, which serve as barriers against invading pathogens (S. S. Gropper et al., 2009; Stephensen, 2001).

Hearing loss and ear infections are common in the developing world and may be related to gestational VAD (Emmett \& West, 2014). Due to vitamin A's relationship with infections and the immune system, a fetal environment that is deficient in vitamin A may lead to ear infections and hearing loss in the child. It is hypothesized that proper fetal development of the inner ear is dependent on adequate vitamin A status during the mother's pregnancy (Emmett \& West, 2014).

The nutrient status of a newborn is highly dependent on the mother's nutrient status. A vitamin supplementation trial done on HIV-infected pregnant and lactating women in Tanzania found that infants born to mothers who received vitamin A had significantly higher serum vitamin A concentrations during their first six months of life compared to infants whose mothers did not receive vitamin A (Table 2.2) (Baylin, Villamor, Rifai, Msamanga, \& Fawzi, 2005). 
Table 2.2. Effects of supplementing HIV-infected pregnant and lactating women on the mean serum concentrations of retinol in their infants at 6 weeks and 6 months of age. (Adapted from Baylin et al., 2005).

\begin{tabular}{lcc|ccc}
\hline \multicolumn{4}{c}{ No vitamin $\boldsymbol{A}$} & \multicolumn{3}{c}{ Vitamin $\boldsymbol{A}$} \\
\hline & $\mathrm{N}$ & Mean $(\mathrm{SD})$ & $\mathrm{N}$ & Mean $(\mathrm{SD})$ & P-value \\
\hline $\begin{array}{l}\mathbf{6} \text { weeks } \\
\text { Retinol } \\
(\mu \mathrm{mol} / \mathrm{L})\end{array}$ & 297 & $0.43(0.16)$ & 297 & $0.52(0.18)$ & $<0.0001$ \\
$\begin{array}{l}\mathbf{6} \text { months } \\
\text { Retinol } \\
(\mu \mathrm{mol} / \mathrm{L})\end{array}$ & 276 & $0.48(0.19)$ & 284 & $0.54(0.21)$ & 0.0002 \\
\hline
\end{tabular}

Pregnant women who are HIV-infected with VAD are at increased risk of having a LBW infant and have increased risk of mother-to-child transmission (MTCT) of HIV (Semba et al., 1995). Infant mortality rates are also greater in women who are deficient in vitamin A. HIV-infected women in Malawi who were severely deficient in vitamin A $(<0.35 \mu \mathrm{mol} / \mathrm{L})$ during pregnancy experienced an infant mortality rate of $93.3 \%$ in their offspring, while women with the most replete vitamin A levels $(>1.75 \mu \mathrm{mol} / \mathrm{L})$ had a $14.2 \%$ infant mortality rate $(\mathrm{p}<0.0001)$ (Semba et al., 1995). In a study in Malawi, HIVinfected pregnant women were randomized to receive a daily dose of 10,000 IU of vitamin A or a placebo from 18-28 weeks gestation until delivery (Kumwenda et al., 2002). Infants born to mothers in the vitamin A group had significantly higher birth weights, and weight and length at 6 weeks of age, compared to infants born into the placebo group. However, by 6 months of age there were no significant differences between the weights and lengths of the two groups. In addition, at 6 weeks of age, infants in the vitamin A group had significantly higher hemoglobin levels and significantly lower rates of anemia, compared to infants born into the placebo group. The results from this study indicate that vitamin A supplementation of 10,000 IU per day during pregnancy can 
result in increased birth weight and growth at 6 weeks, and reduced prevalence of anemia in infants born in HIV-infected mothers (Kumwenda et al., 2002).

Vitamin A may have an effect on lowering anemia rates through a variety of possible mechanisms, including the ability to mobilize iron stores from tissues, to reduce anemia of infection, and to increase growth of precursor cells that differentiate into erythrocytes (red blood cells) (Kumwenda et al., 2002; Semba et al., 2001). A study involving pregnant women in Indonesia found that vitamin A combined with iron supplementation reduced rates of anemia more than iron on its own (Suharno, West, Muhilal, Karyadi, \& Hautvast, 1993). Vitamin A may play a role in reducing iron deficiency anemia through improving resistance to infections, regulating red blood cell production, and influencing iron metabolism and absorption (Michelazzo, Oliveira, Stefanello, Luzia, \& Rondó, 2013).

Despite evidence from singular studies showing associations between prenatal vitamin A supplementation and improved maternal and infant outcomes, a 2011 Cochrane review and meta-analysis of 16 randomized controlled trials of maternal vitamin A supplementation (van den Broek et al., 2011) found no significant effect on the relative risk of maternal mortality (Green, Pindar, Davis, \& Mellanby, 1931; Kirkwood et al., 2010; West et al., 1999), perinatal mortality (Kirkwood et al., 2010), neonatal mortality (Coutsoudis, Pillay, Spooner, Kuhn, \& Coovadia, 1999; Kirkwood et al., 2010; Kumwenda et al., 2002; West et al., 1999), stillbirths (Kirkwood et al., 2010; Kumwenda et al., 2002; Muslimatun et al., 2001), neonatal anemia (Fawzi et al., 1998; Kumwenda et al., 2002), preterm births (Coutsoudis et al., 1999), or LBW infants (Cox et al., 2005; Kumwenda et al., 2002; Radhika, Bhaskaram, Balakrishna, \& Ramalakshmi, 2003). 
However, antenatal vitamin A supplementation did show a significant reduction in the risk of night blindness (West et al., 1999) and maternal anemia (Fawzi et al., 1998; Muslimatun et al., 2001; Semba et al., 2001; Suharno et al., 1993; West et al., 1999). There was a reduced risk of LBW infants from HIV-infected women when comparing vitamin $\mathrm{A}$, iron and folate supplementation to iron and folate alone (RR 0.67, 95\% CI 0.47, 0.96) (Kumwenda et al., 2002). Some evidence was found that vitamin A supplementation might reduce the risk of maternal clinical infections. These authors conclude that there may not be a role for antenatal vitamin A supplementation to reduce maternal or infant mortality, but there is strong evidence that vitamin A supplementation can reduce maternal anemia, especially in HIV-infected populations (van den Broek et al., 2011). A 2015 Cochrane review including 19 trials found similar results and came to the same conclusions as the 2011 review (McCauley, van den Broek, Dou, \& Othman, 2015).

\subsubsection{Vitamin D}

Vitamin D is a fat-soluble seco-steroid that is sometimes referred to as a prohormone rather than a vitamin, because it has the ability to be synthesized under the skin from sunlight, therefore it is not essential to consume. Vitamin D has long been associated with effects on the bone and its role in maintaining calcium concentrations in the body. Recent research has begun to associate vitamin D with many more functions. During pregnancy, a wide array of diseases and complications for both mother and fetus have been linked to low levels of vitamin D (Christakos et al., 2013).

The traditional roles of vitamin D in the body involve bone health and calcium homeostasis. However, vitamin D has recently been discovered to have an impact on 
almost all body systems. Vitamin D receptors (VDR) have been found in 37 human tissues in both the classical systems (bone, kidney, and intestine), as well as unexpected cells such as in the pancreas, placenta, brain, skin, cardiovascular, and immune systems. This means that vitamin D, or lack thereof, may have an effect on many different diseases and conditions (Barrett \& McElduff, 2010; Christakos et al., 2013).

There are few naturally occurring dietary sources of vitamin D, including fatty fish (salmon, tuna, herring, sardines, and fish liver oils), liver, beef, veal, egg yolks, and cheese. In the United States, fortified vitamin D products include milk, margarine, orange juice, and some breads and breakfast cereals (S. S. Gropper et al., 2009). In Malawi, oils and skimmed milk powder are fortified with vitamin D (World Food Programme, 2008a).

In populations living in areas of adequate sunshine, vitamin $\mathrm{D}$ levels are mostly affected by sunlight, rather than dietary intake of vitamin D. The bioavailability and synthesis of vitamin D depends on various factors, including season, time of day, skin color, latitude, type of clothing, use of sunscreen, obesity, and other health conditions (Gebreegziabher \& Stoecker, 2013). Individuals with darker skin need a longer time in the sun in order to make adequate amounts of vitamin D due to higher concentrations of melanin in the skin, compared to lighter skinned people. Melanin is a large polymer that competes with 7-dehydrocholesterol for UVB photons, thus demanding a longer time in the sun to generate the same amount of vitamin D in the skin (Tsiaras \& Weinstock, 2011).

Vitamin D deficiency causes decreased calcium and phosphorus absorption, which stimulates parathyroid hormone (PTH) secretion to remove calcium from the bone in order to maintain calcium homeostasis. This results in decreased calcium in the bones 
and secondary hyperparathyroidism (Pettifor \& Prentice, 2011; Uriu-Adams, Obican, \& Keen, 2013).

No large-scale studies of VDD in Malawian pregnant women have been reported. A comparison study from 2013 assessed VDD in Malawian adults in the hospital, with or without tuberculosis (TB) infection (Mastala et al., 2013). The mean 25(OH)D level in non-TB patients was $84.2 \mathrm{nmol} / \mathrm{L}$, which was significantly higher than the mean 25(OH)D level of TB patients $(59.7 \mathrm{nmol} / \mathrm{L})$ (Mastala et al., 2013). Patients with TB also had significantly higher rates of low vitamin D levels compared to non-TB patients (74.5\% and $47.8 \%$, respectively) and VDD (42.2\% and $16.6 \%$, respectively). In this study, low BMI and antiretroviral use (in HIV patients) were associated with lower vitamin D levels. Low BMI may be a risk factor for VDD because the lack of adipose tissue may result in an inability to store vitamin D (Mastala et al., 2013).

Assessment of VDD is complicated by the fact that there is no standardized cutoff value for deficiency (Peragallo Urrutia \& Thorp, 2012). Differing serum concentration categorizations of $25(\mathrm{OH}) \mathrm{D}$ are found in Table 2.3.

Table 2.3. Serum 25(OH)D concentrations and deficiency cut-off values.

\begin{tabular}{llll}
\hline & Sufficiency & Insufficiency & Deficiency \\
\hline NIH $^{1}$ & $>20 \mathrm{ng} / \mathrm{mL}$ & $12-20 \mathrm{ng} / \mathrm{mL}$ & $<12 \mathrm{ng} / \mathrm{mL}$ \\
& $>50 \mathrm{nmol} / \mathrm{L}$ & $30-50 \mathrm{nmol} / \mathrm{L}$ & $<30 \mathrm{ng} / \mathrm{mL}$ \\
\hline ARUP Reference & $>30 \mathrm{ng} / \mathrm{mL}$ & $20-29 \mathrm{ng} / \mathrm{mL}$ & $<20 \mathrm{ng} / \mathrm{mL}$ \\
Laboratory $^{2}$ & $>75 \mathrm{nmol} / \mathrm{L}$ & $50-72 \mathrm{nmol} / \mathrm{L}$ & $<50 \mathrm{nmol} / \mathrm{L}$ \\
\hline Gebreegziabher, et $^{2}$ & $>20 \mathrm{ng} / \mathrm{mL}$ & $\mathrm{N} / \mathrm{A}$ & $<12 \mathrm{ng} / \mathrm{mL}$ \\
al $(2013)^{3}$ & $>50 \mathrm{nmol} / \mathrm{L}$ & & $<30 \mathrm{nmol} / \mathrm{L}$ \\
& & & \\
\hline ACOG $(2011)^{4}$ & $>20 \mathrm{ng} / \mathrm{mL}$ & $\mathrm{N} / \mathrm{A}$ & $<20 \mathrm{ng} / \mathrm{mL}$ \\
& $>50 \mathrm{nmol} / \mathrm{L}$ & & $<50 \mathrm{nmol} / \mathrm{L}$ \\
\hline
\end{tabular}

${ }^{1}$ (National Institutes of Health, 2014)

${ }^{2}$ ARUP Laboratories (2016)

${ }^{3}$ (Gebreegziabher \& Stoecker, 2013)

${ }^{4}$ (ACOG Committee on Obstetric Practice, 2011) 
Vitamin D insufficiency during pregnancy has been linked to adverse infant outcomes, including LBW and SGA infants, neonatal hypocalcemia, low bone mineral density, and impaired growth (Barrett \& McElduff, 2010). Infants born to mothers with VDD are more likely to develop autoimmune diseases, asthma, allergies, and wheezing later in life (Barrett \& McElduff, 2010). A meta-analysis of 31 studies found that insufficient levels of vitamin D were associated with gestational diabetes, preeclampsia, and SGA newborns. Mothers with 25(OH)D levels below $37.5 \mathrm{nmol} / \mathrm{L}$ had lower birth weight infants (by an average of $131 \mathrm{~g}$ ), compared to mothers with 25(OH)D levels above $37.5 \mathrm{nmol} / \mathrm{L}$ (Aghajafari et al., 2013). Another recent meta-analysis found that supplementing with vitamin $\mathrm{D}_{3}$ during pregnancy resulted in a $60 \%$ reduced risk of having a LBW newborn (RR 0.40, 95\% CI 0.23-0.71) (Thorne-Lyman \& Fawzi, 2013).

Infants who are born to mothers with inadequate levels of vitamin D are more likely to have increased rates of premature birth compared to infants born to mothers with adequate vitamin D stores (Bodnar et al., 2013). A cohort study of women with twin pregnancies assessed vitamin D concentrations and rates of premature deliveries. Mothers with $25(\mathrm{OH}) \mathrm{D}$ levels above $75 \mathrm{nmol} / \mathrm{L}$ were $60 \%$ less likely to have a preterm birth, compared to women with 25(OH)D levels less than $75 \mathrm{nmol} / \mathrm{L}$ (Bodnar et al., 2013). A trial in India randomized pregnant women to receive either one dose of $1500 \mu \mathrm{g}$ vitamin $\mathrm{D}_{3}$ in their second trimester, one dose of $3000 \mu \mathrm{g}$ vitamin $\mathrm{D}_{3}$ in both the second and third trimesters, or no vitamin D treatment (Kalra et al., 2012). Both of the vitamin D groups had infants with significantly higher birth weights, lengths, and head circumferences compared to the control group infants (Kalra et al., 2012). A randomized controlled trial in Bangladesh supplied pregnant women with 35,000 IU of vitamin D per week from the 
third trimester until delivery or a placebo (Roth, Perumal, Al Mahmud, \& Baqui, 2013). While there were no significant differences between the anthropometric measurements at birth, length-for-age Z-scores (LAZ) and growth rates at 1 month of age were significantly higher in the infants in the vitamin $\mathrm{D}$ group. For every $10 \mathrm{nmol} / \mathrm{L}$ increase in maternal 25(OH)D level at delivery, there was a $0.06 \mathrm{LAZ}$ increase at 1 month of age. Inadequate linear growth during infancy can lead to childhood stunting, which has longterm health consequences (Roth et al., 2013). Lower birth lengths and infant growth rates are also associated with increased risk of bone fractures in later life (Zhu et al., 2013).

Vitamin D is known to modulate the immune system by increasing production of antimicrobial peptides (AMPs) and anti-inflammatory cytokines (Mehta et al., 2010). Cathelicidin, an AMP, is increased in the presence of adequate $25(\mathrm{OH}) \mathrm{D}$, which can lead to increased antimicrobial action against pathogens. Another proposed mechanism is that vitamin $\mathrm{D}$ increases phagocytosis by macrophages and increases production of natural killer cells, which target invading pathogens (Mehta et al., 2010, 2011).

HIV-infected individuals are susceptible to contracting opportunistic infections, with the most common being TB (Mehta et al., 2010). Vitamin D has been shown to fight or prevent TB infections by the process of increasing AMPs, such as cathelicidin. TB is a common cause of mortality among HIV-infected individuals, thus adequate vitamin D status may reduce risk of mortality from opportunistic infections (Mehta et al., 2010). Vitamin D is crucial for proper function of many body tissues and cells, and a lack of it may result in increased risk of adverse maternal and neonatal outcomes. 


\subsubsection{Vitamin $B_{12}$}

Vitamin $\mathrm{B}_{12}$, or cobalamin, is actually a group of water-soluble compounds called corrinoids that have a cobalt molecule in the center of a corrin nucleus (FAO \& WHO, 1998). Dietary vitamin $B_{12}$ is found only in animal products because no plant species have the required enzymes for vitamin $\mathrm{B}_{12}$ synthesis. Bacteria, algae, and mammalian cells have enzymes that are dependent on vitamin $\mathrm{B}_{12}$ to function. Vitamin $\mathrm{B}_{12}$ is present in animal products because it is synthesized in microorganisms that enter the gastrointestinal (GI) tract of animals, and vitamin $\mathrm{B}_{12}$ then gets absorbed and incorporated into their tissues (FAO \& WHO, 1998). The best sources of vitamin $\mathrm{B}_{12}$ are meat, poultry, fish, clams, oysters and eggs. Some plant foods are fortified with vitamin $B_{12}$ (S. S. Gropper et al., 2009).

Vitamin $\mathrm{B}_{12}$ plays a role in DNA synthesis by converting homocysteine to methionine, using methylcobalamin as the coenzyme. A lack of vitamin $\mathrm{B}_{12}$ will result in a buildup of homocysteine (S. S. Gropper et al., 2009). High levels of homocysteine have been linked to oxidative stress, increased risk of cardiovascular disease, placental abruption, stillbirth and preterm delivery, LBW infants, and preeclampsia (Allen, 2005; Lonn et al., 2006). Vitamin $B_{12}$ also acts as a cofactor in the conversion of Lmethylmalonyl-CoA to succinyl CoA. Low levels of vitamin $\mathrm{B}_{12}$ will then result in a buildup of methylmalonic acid, which has detrimental consequences to fetal development. Children with genetic mutations who are deficient in the enzyme to perform this conversion are born with mental retardation and other developmental defects (Duggan et al., 2014). When serum vitamin $B_{12}$ levels are inadequate, first DNA synthesis is decreased, followed by an increase in serum homocysteine and 
methylmalonic acid concentrations, and finalizing in cell changes seen in megaloblastic anemia. Megaloblastic macrocytic anemia is characterized by large, immature red blood cells. Symptoms include fatigue, shortness of breath, pale skin, heart palpitations, insomnia, tingling of the extremities, loss of concentration and memory, disorientation, and dementia (S. S. Gropper et al., 2009).

Vitamin $\mathrm{B}_{12}$ and folate have a complex interaction with one another. The enzyme methionine synthase is dependent on vitamin $\mathrm{B}_{12}$ and functions in the methylation cycle, which also requires folate. A disruption of this cycle (due to either folate or vitamin $B_{12}$ deficiency) will result in reduced levels of myelin proteins, causing demyelination of peripheral nerves and the spinal column. Nerve demyelination can lead to neuropathy involving paralysis, ataxia, and even death if untreated. A lack of adequate vitamin $\mathrm{B}_{12}$ or folate for this cycle can also cause reduced DNA synthesis and megaloblastic anemia (FAO \& WHO, 1998; Weir \& Scott, 1999).

When an individual has a deficiency in vitamin $\mathrm{B}_{12}$, but not folate, it will result in a pseudo-folate deficiency due to what is called the "methyl trap hypothesis". The first reaction in the methylation cycle that requires folate is irreversible, and the only mechanism by which to convert this folate-containing intermediate product to the final product is through methionine synthase, the enzyme requiring vitamin $\mathrm{B}_{12}$. Without adequate vitamin $\mathrm{B}_{12}$, the body's available folate will be "trapped" in the intermediate stage of this reaction, which results in symptoms of a folate deficiency (FAO \& WHO, 1998; Weir \& Scott, 1999).

A study done in Malawi found that vitamin $\mathrm{B}_{12}$ deficiency affected $16-33 \%$ of pregnant women, depending on the cut-off value that was used (van den Broek \& Letsky, 
2000). Low vitamin $B_{12}$ concentration was significantly correlated with megaloblastic cell changes in bone marrow aspirates $(\mathrm{p}<0.001)$, indicating that vitamin $\mathrm{B}_{12}$ deficiency is a cause of megaloblastic anemia in this population (van den Broek \& Letsky, 2000).

During pregnancy, it is estimated that 0.1-0.2 $\mu \mathrm{g}$ of vitamin $\mathrm{B}_{12}$ can be transferred to the fetus and accumulated in their liver during the second and third trimesters. However, infants born to mothers with inadequate vitamin $\mathrm{B}_{12}$ intake and status may present clinical signs of deficiency, such as neuropathy (FAO \& WHO, 1998). Due to this trans-placental transfer of vitamin $\mathrm{B}_{12}$, there is an observed decrease in maternal serum levels close to term. At the end of pregnancy, it may be difficult to correctly assess vitamin $B_{12}$ levels via serum (van den Broek \& Letsky, 2000).

Inadequate maternal vitamin $\mathrm{B}_{12}$ concentrations can lead to adverse pregnancy and birth outcomes (Allen, 2005). A study from Nepal found that pregnant women who were vitamin $\mathrm{B}_{12}$ deficient had a two-fold increased risk of preeclampsia and preterm delivery (Bondevik et al., 2001). A study in Kenya found that pregnant women with vitamin $\mathrm{B}_{12}$ deficiencies were more likely to have growth restricted infants and stillbirth babies (Neumann et al., 2014). This study also found a negative relationship between maternal vitamin $\mathrm{B}_{12}$ status and newborn neurobehavioral scores. A limited supply of vitamin $\mathrm{B}_{12}$ from the mother to the fetus can lead to impaired fetal growth and brain development, which can translate to cognitive problems later in life (Neumann et al., 2014). A systematic review and meta-analysis found that women with vitamin $B_{12}$ insufficiency during pregnancy had a 1.7 times increased risk of having a SGA or LBW infant (95\% CI: 1.16, 2.50) (Sukumar et al., 2016). 
Infants with vitamin $\mathrm{B}_{12}$ deficiencies are more likely to have anemia, muscle weakness, cognitive disorders, and psychomotor regression (Neumann et al., 2014). Infants who are exclusively breastfed from mothers with inadequate vitamin $B_{12}$ intake are likely to develop a deficiency, because breast milk will be the infant's only source of the vitamin. Elevated homocysteine levels have been linked to these adverse outcomes in fetal growth and development (Neumann et al., 2014).

A randomized controlled trial in India aimed to discover if maternal vitamin $B_{12}$ supplementation had an impact on infant vitamin $\mathrm{B}_{12}$ status and birth outcomes (Duggan et al., 2014). The study randomized 183 women to receive $50 \mu \mathrm{g}$ vitamin $\mathrm{B}_{12}$ and 183 women to receive a placebo. Women who took vitamin $\mathrm{B}_{12}$ supplements had higher serum vitamin $\mathrm{B}_{12}$ concentrations during the third trimester, as well as higher content in their breast milk. Infants born to mothers in the vitamin $\mathrm{B}_{12}$ group had higher serum concentrations of the vitamin, as well as lower concentrations of plasma homocysteine and methylmalonic acid. This study found no difference between the two groups in terms of birth weight or prevalence of IUGR, although the authors noted that the study was inadequately powered to detect a difference in these outcomes. This study indicated that supplementing pregnant women with vitamin $\mathrm{B}_{12}$ can elicit a response in the vitamin $\mathrm{B}_{12}$ concentration of their infants, which can have positive effects on the infant's growth (Duggan et al., 2014).

Low vitamin $B_{12}$ status in a pregnant woman can have important consequences for both herself and her fetus. Studies have shown that maternal supplementation of vitamin $\mathrm{B}_{12}$ can improve the vitamin $\mathrm{B}_{12}$ status of infants, which can positively impact an infant's development. A mother who has adequate vitamin $\mathrm{B}_{12}$ has a lower risk of anemia, 
preeclampsia, and adverse birth outcomes, such as delivering a newborn who is LBW, IUGR, preterm, or stillbirth (Duggan et al., 2014).

\subsubsection{Folate/folic acid}

Folate is the term used for the water soluble B-vitamin found in its reduced form in body tissue and naturally in foods (S. Gropper, Smith, \& Groff, 2009). Food sources of folate include green vegetables, fruits, mushrooms, peanuts, legumes, lentils, and liver. The bioavailability of folate from foods can vary greatly, from $10-98 \%$, based on genetic differences, intestinal $\mathrm{pH}$, and various food inhibitors of folate absorption (S. S. Gropper et al., 2009; Sauberlich, 1985).

The RDA for folate for women who are pregnant or capable of becoming pregnant is set at $600 \mu \mathrm{g}$ DFE, whereas the RDA for adults is set at $400 \mu \mathrm{g}$ DFE. It is recommended that pregnant women (or women planning to become pregnant) take a supplement containing $400 \mu \mathrm{g}$ of folic acid and consume folate-containing foods (Food and Nutrition Board, 1998; S. S. Gropper et al., 2009).

Absorption of folate is impaired by zinc deficiency, chronic alcohol use, and foods such as cabbage, oranges, lentils, and legumes; these will reduce digestion and absorption of folate from foods (S. Gropper et al., 2009). Folate functions in the cytoplasm and mitochondria as a coenzyme, making it essential for DNA synthesis, cell

division and nucleotide synthesis. Folate is involved in the metabolism of the amino acids histidine, serine, glycine, and methionine. If there is inadequate folate in the body, homocysteine concentrations will increase, as discussed in section 2.2.3 (S. S. Gropper et al., 2009). 
Folate deficiency can result in megaloblastic macrocytic anemia, similar to a vitamin $\mathrm{B}_{12}$ deficiency. Initially, a folate deficiency will present with low plasma folate after approximately one month of low folate intake, followed by low red blood cell folate concentrations after 3-4 months (Hine, 1993). The symptoms of megaloblastic anemia occur after 4-5 months of deficient folate intake and are discussed in the previous section 2.2.3. Red blood cells become enlarged (megaloblastic) because of abnormal DNA synthesis and the inability of blood cells to divide, combined with the continued formation of RNA. This results in an excess production of RNA and other cell components, such as hemoglobin, causing immature and large red blood cells (S. S. Gropper et al., 2009).

Pregnant women are at increased risk of developing a folate deficiency because fetal growth increases the demands for folate, especially in the second and third trimesters (McPartlin, Halligan, Scott, Darling, \& Weir, 1993). Folate deficiencies during pregnancy are well known to cause fetal neural tube defects (NTDs). The effects of folate deficiency take place in early pregnancy, which is why it is crucial for all women who are capable of becoming pregnant to take folic acid supplements. The fetal neural plate closes to form the spinal cord and cranium between days 21 and 27 post-conception. If a woman has inadequate folate concentrations during this time, the spinal cord and/or cranium will not close properly, resulting in spina bifida and/or anencephaly, respectively (FAO \& WHO, 1998). Maternal folate deficiency can also cause other congenital malformations, including orofacial clefts and cardiac defects, as well as pregnancy complications, such as preeclampsia, IUGR, bleeding and miscarriages (Black, 2001; Hovdenak \& Haram, 2012). 
Recent data on maternal folate deficiency in Malawi are lacking. A study from 2000 analyzed causes of anemia in pregnant women in Malawi (van den Broek \& Letsky, 2000). Thirty-four percent of the anemic women in this study population were also found to have a folate deficiency. Folate deficiency may also be worsened by malarial infection (van den Broek \& Letsky, 2000).

It is recommended that women take a $400 \mu \mathrm{g}$ folic acid supplement preconception and through the first trimester of pregnancy (Centers for Disease Control, 1992; WHO, 2012a). However, recommendations for the second and third trimesters are not as clear. Women who took the recommended amount of folic acid during their first trimester were included in a study to determine whether there is a benefit to taking 400 $\mu \mathrm{g}$ folic acid throughout the entire pregnancy (Mcnulty et al., 2013). Women in the placebo group had significantly lower serum and red blood cell folate levels and significantly higher homocysteine levels, compared to women who continued to take folic acid. Infants born to mothers in the folic acid group had significantly higher cord blood levels of folate. High homocysteine levels during pregnancy have been linked to preeclampsia, premature delivery, and lower birth weight babies, therefore it may be beneficial for women to continue to take folic acid throughout the entire pregnancy (Mcnulty et al., 2013; Vollset et al., 2000).

Fetal development and maternal health are both associated with adequate folate concentrations during pregnancy. Consequences of maternal folate deficiency include high risk of fetal NTD's, high homocysteine levels and adverse pregnancy outcomes. It is imperative that women of reproductive age take folic acid supplements in order to 
prevent these consequences, primarily congenital abnormalities of the fetus (FAO \& WHO, 1998; Hovdenak \& Haram, 2012).

\subsubsection{Iron}

Iron is a mineral that has several essential functions in the human body, including being a carrier of oxygen to tissues via hemoglobin. The average person has between 2 and 4 grams of iron in their body, which is dependent on gender, body weight, age and pregnancy. The only forms of iron that are in food and the body are the ferric $\left(\mathrm{Fe}^{3+}\right)$ and ferrous $\left(\mathrm{Fe}^{2+}\right)$ forms (FAO \& WHO, 1998; S. S. Gropper et al., 2009).

There are two forms of iron in the diet, heme and non-heme (S. S. Gropper et al., 2009). The primary difference between the two is that heme iron is derived from the hemoglobin and myoglobin in animal products, especially meat, poultry, and fish. Nonheme iron is found in plant foods (such as grains, tofu, nuts, vegetables and fruits) and in small amounts in dairy products. The best sources of iron include liver, red meat, organ meats, clams, oysters, and beans. Iron is also fortified into some breads, pastas, cereals, and flours (S. S. Gropper et al., 2009).

There are several factors that can inhibit or enhance iron absorption. Compounds that bind with non-heme iron are known as chelates or ligands. A chelate/ligand complex with non-heme iron will either bind too strongly to it, resulting in excretion of iron, or it will bind loosely, enhancing absorption. The dietary factors that can either enhance or inhibit absorption are found in Table 2.4. Phytates are salts that store phosphates and minerals and are found in fruits, vegetables, grains and cereals, seeds, nuts, and roots. Zinc and iron negatively impact each other's absorption by competing for the same transporters. Iron absorption is also linked to an individual's body iron stores. People 
with iron deficiencies may absorb up to $35 \%$, whereas an individual with normal iron status will absorb only 10\% (FAO \& WHO, 1998; S. S. Gropper et al., 2009; Hallberg, Hulten, \& Gramatkovski, 1997).

Table 2.4. Dietary factors that can inhibit or enhance absorption of iron (FAO \& WHO, 1998; S. S. Gropper et al., 2009).

\begin{tabular}{l|l}
\hline \multicolumn{1}{c|}{ INHIBITING FACTORS } & \multicolumn{1}{c}{ ENHANCING FACTORS } \\
\hline $\begin{array}{l}\text { Phytates (whole grains, bran, legumes, corn, } \\
\text { soybeans, rice) }\end{array}$ & $\begin{array}{l}\text { Ascorbic acid/vitamin C (fruits and some } \\
\text { vegetables) }\end{array}$ \\
$\begin{array}{l}\text { Polyphenols and tannins (tea and coffee) } \\
\text { Oxalic acid (spinach, chocolate, berries, tea) }\end{array}$ & Meat, poultry, fish, and seafood \\
$\begin{array}{l}\text { Calcium, calcium-phosphate salts, zinc, } \\
\text { nickel, manganese }\end{array}$ & Fermented foods \\
\hline
\end{tabular}

Humans have unique methods for attempting to prevent iron deficiency and overload. Erythrocytes die after approximately 120 days, and the body will reutilize the iron from these spent red blood cells by bringing the iron back to other tissues or to erythrocyte precursors (FAO \& WHO, 1998). This mechanism is also important to reduce the amounts of free iron circulating in the blood, because unbound iron can lead to free radicals, which are damaging to cells. Free iron is also readily used by bacteria for growth and multiplication, which can cause or worsen infections (S. S. Gropper et al., 2009). Another method to maintain iron balance involves ferritin, the storage protein, which will create an iron reservoir in times of increased iron demands, such as during pregnancy. The intestines also play a role in maintaining iron balance by increasing absorption during times of low body iron stores and reducing absorption when iron levels are high (FAO \& WHO, 1998). 
The RDA for iron for premenopausal women is $18 \mathrm{mg}$ per day, whereas the RDA for men and postmenopausal women is $8 \mathrm{mg}$ per day. During pregnancy, the RDA increases to $27 \mathrm{mg}$ per day. While pregnant women do not lose menstrual blood, they still require a higher amount of iron because additional iron is needed for the fetus, placenta, increased plasma volume, and for a storage reservoir for blood loss during childbirth, as seen in Table 2.5 (Food and Nutrition Board, 2001). The World Health Organization recommends a dose of 30-60 $\mathrm{mg}$ per day during pregnancy, and that it should be $60 \mathrm{mg}$ in areas where anemia is a severe public health problem (affecting $>40 \%$ of the population) (WHO, 2012a).

Table 2.5. Iron requirements and losses during a healthy pregnancy and delivery. Adapted from (FAO \& WHO, 1998).

\begin{tabular}{l|l}
\hline Iron Needs for Pregnancy and Delivery & Iron requirements or losses (mg) \\
\hline Fetus & 300 \\
Placenta & 50 \\
Maternal plasma expansion & 450 \\
Basal iron losses & 240 \\
\hline Total Additional Iron Requirement & $\mathbf{1 0 4 0}$ \\
\hline IRON BALANCE AFTER DELIVERY & +450 (back to mother) \\
Contraction of maternal RBC mass & -250 \\
Maternal blood loss & +200 \\
Net iron balance after delivery & $\mathbf{8 4 0}$ (1040-200) \\
\hline $\begin{array}{l}\text { NET IRON REQUIREMENTS FOR } \\
\text { PREGNANCY* }\end{array}$ &
\end{tabular}

Globally, iron is the most common nutrient deficiency in pregnant women (Ramakrishnan, 2002). A deficiency in iron can be with or without anemia, which doesn't 
occur until iron levels are severely depleted (S. S. Gropper et al., 2009). The criteria for anemia of pregnant women is usually defined as a hemoglobin concentration of $<10.5$ or $11.0 \mathrm{~g} / \mathrm{dL}$ (van den Broek \& Letsky, 2000). As opposed to the megaloblastic macrocytic anemia seen with folate or vitamin $\mathrm{B}_{12}$ deficiencies, iron deficiency anemia presents with microcytic and hypochromic red blood cells (S. S. Gropper et al., 2009). While there are multiple potential causes of anemia, iron deficiency is the most common, accounting for approximately 50\% of all anemia cases (Klemm, Sommerfelt, Boyo, \& Barba, 2011; Sangaré, van Eijk, Ter Kuile, Walson, \& Stergachis, 2014). During pregnancy, hemoglobin and hematocrit concentrations are known to decline throughout the first and second trimesters, followed by an increase in the third trimester (Scholl, 2005). Plasma ferritin concentrations can also be used to determine iron deficiency before anemia is present. Reported cut-off values for deficiency vary from 12.0-15.0 $\mu \mathrm{g} / \mathrm{L}(\mathrm{FAO} \& \mathrm{WHO}$, 1998; S. S. Gropper et al., 2009). However, ferritin is a positive acute phase protein, thus if inflammation or infection are present, ferritin levels will be falsely elevated (S. S. Gropper et al., 2009). A method to combat this problem is to also take measurement of an acute phase protein, such as C-reactive protein or $\alpha$-1-acid glycoprotein (AGP) (EngleStone, Nankap, Ndjebayi, Erhardt, \& Brown, 2013).

Symptoms of iron deficiency include pale skin, reduced behavioral and cognitive abilities, fatigue, cold extremities, and short attention span (Scrimshaw, 1991). These symptoms are related to the reduced transport of oxygen (via hemoglobin) to tissues in the body (S. S. Gropper et al., 2009).

Anemia is a major cause of maternal and fetal morbidity and mortality, as well as adverse pregnancy outcomes (Scholl, 2005). Pregnant women require greater amounts of 
iron to meet the needs of the placenta and fetus. Women of reproductive age in developing countries tend to have a diet that is inadequate to meet these increased iron needs. Women with higher parity, which is common in developing countries, have increased risk of iron deficiency (Scholl, 2005).

The iron stores of a woman at the time of conception are thought to be a strong predictor of her iron status and anemia risk during pregnancy. It can be hard to replenish inadequate iron stores once pregnancy has begun (Allen, 2005). The placenta regulates how much iron is transferred from mother to fetus. When maternal iron levels are low, the number of receptors on the placenta will increase, in order to take up more iron for the fetus. The majority of iron transfer from mother to fetus occurs during the third trimester, typically after the $30^{\text {th }}$ week (Allen, 2000).

It has been estimated that for every $1.0 \mathrm{~g} / \mathrm{dL}$ increase in hemoglobin concentrations in anemic pregnant women, there is a $20 \%$ reduction in risk of maternal mortality (Klemm et al., 2011). Maternal iron deficiency can result in increased risk of preterm delivery, neonatal and infant iron deficiency, LBW infants, stillbirths, and poor cognitive fetal development (Allen, 2005; Bhutta et al., 2012; Scholl, 2005). Iron deficiency anemia (IDA) is thought to contribute to preterm delivery through three potential mechanisms: hypoxia due to reduced oxygen transport, oxidative stress, and increased susceptibility to infections (Scholl, 2005). Oxidative stress is more likely to occur in mother-fetus pairs with iron deficiency because IDA leads to increased cortisol production, which can damage fetal red blood cells. Damaged erythrocytes can result in decreased fetal growth (Hovdenak \& Haram, 2012). Infants born preterm are more likely to have perinatal complications and have stunted growth later on in life (Allen, 2000). 
Children born to mothers who had inadequate iron stores during pregnancy are at increased risk of having lower cognitive, motor, and socio-emotional development skills (Prado \& Dewey, 2014). Iron deficiency has an impact on neurodevelopment of the fetus due to a lack of hemoglobin, which transports oxygen to the brain (Prado \& Dewey, 2014). Maternal iron deficiency also leads to decreased iron transfer to the fetus, which can have an impact on the function of the developing brain (McArdle, Gambling, \& Kennedy, 2014).

Of 4,646 pregnant women attending a hospital in southern Malawi, 57\% of them were found to be anemic, with deficiency in iron being the primary cause of the anemia. However, multiple micronutrient deficiencies were also common in this population. Sixteen percent of the anemic women were found to be severely anemic, with hemoglobin levels $<7 \mathrm{~g} / \mathrm{dL}$ (van den Broek \& Letsky, 2000).

Prenatal iron supplementation is a global public health measure in place to prevent maternal anemia. Maternal iron supplementation generally improves iron status within 3 months; however, women who begin pregnancy with iron deficiency may not be able to regain adequate iron status if iron is not taken from the start of pregnancy. Compliance with daily supplementation throughout the entire pregnancy can result in better iron stores for up to 2 months postpartum (Allen, 2000). It is important that maternal iron stores remain adequate after delivery because women in developing countries tend to have shorter intervals between pregnancies; thus, they will be more likely to enter a new pregnancy with better iron stores if iron supplements were taken throughout the previous pregnancy. Although maternal and cord blood ferritin tend to be closely related, maternal and cord blood hemoglobin are independent, unless the mother 
is severely anemic. Infants who are born to anemic mothers are more likely to be anemic as well (Allen, 2000). In a Cochrane review, maternal iron supplementation was found to reduce anemia risk by $70 \%$, reduce iron deficiency at term by $57 \%$, reduce risk of LBW infants, and improve neonatal and infant iron stores (Pena-Rosas, De-Regil, Dowswell, \& Viteri, 2012).

While the evidence supporting prenatal iron supplementation is strong, there are also studies reporting the harmful effects of over-supplementation of iron. In one study, women with ferritin levels above $41 \mathrm{ng} / \mathrm{mL}$ during pregnancy had increased risk of preterm delivery, possibly due to intrauterine infection (Goldenberg et al., 1996). There is concern that taking additional iron supplements in non-anemic women may result in symptoms of iron overload. Excessive maternal iron stores may impair blood flow to the placenta due to increased blood viscosity (Rush, 2000; Scholl, 2005). A study found that women with high hemoglobin in the first and second trimesters had higher risk of having SGA births, but not preterm births (Scanlon, Yip, Schieve, \& Cogswell, 2000). A Ushaped association has been reported between maternal hemoglobin levels and birth weight of infants (Mahomed, 2007). Both low $(<8.0 \mathrm{~g} / \mathrm{dL})$ and very high $(>12.0 \mathrm{~g} / \mathrm{dL})$ hemoglobin concentrations may lead to LBW infants (Scholl, 2005). Other issues associated with high maternal iron stores include increased risk of preeclampsia and gestational diabetes mellitus. Excess free iron in contact with oxygen can create harmful free radicals in the body, which damage cells and tissues. This oxidative damage and stress may contribute to increased risk of preterm delivery (Scholl, 2005).

Another concern involving high iron stores involves the relationship between iron and malaria. Each year, 25 to 30 million women become pregnant in areas of Africa that 
are malaria-endemic (Rogerson, Mwapasa, \& Meshnick, 2007; Steketee, Nahlen, Parise, \& Menendez, 2001). Malaria can cause severe anemia during pregnancy, resulting in 10,000 maternal deaths per year worldwide (Guyatt \& Snow, 2001). Malaria also contributes to $75,000-200,000 \mathrm{LBW}$ babies born each year, due to a combination of IUGR and preterm deliveries (Guyatt \& Snow, 2004; Rogerson et al., 2007). A placenta that is infected with malaria activates an immune response, which may be responsible for stimulating an early labor (Guyatt \& Snow, 2004). The presence of free iron in the body may increase susceptibility to malarial infection by increasing growth of pathogens (Senga, Harper, Koshy, Kazembe, \& Brabin, 2011). The malaria parasite requires iron for its survival, growth and proliferation (Sangaré et al., 2014). A case-control study in Malawi found that pregnant women with placental malaria had better iron stores than women without malaria. Iron deficiency may limit the availability of iron to the malaria parasite, thus reducing infection risk (Senga et al., 2011). Malaria can then cause anemia through removal of excessive numbers of red blood cells, immune destruction, and impaired red blood cell production (Ekvall, 2003; Ouma et al., 2007). Women in their first pregnancy tend to have higher malaria infection rates. This may be due to younger age, or the fact that multigravida women are more likely to be iron deficient due to compounding effects of iron requirements during pregnancy (Rogerson et al., 2007; Senga et al., 2011).

A meta-analysis found that iron supplementation did not significantly increase risk of malaria (P. falciparum) infection during pregnancy or at delivery (Sangaré et al., 2014). Women with low ferritin levels had a reduced risk of malaria; however, there was no association between malaria risk and other biomarkers of iron deficiency. The authors 
conclude that iron supplementation does not increase malaria risk during pregnancy if the women are concurrently using malaria prevention strategies, such as insecticide-treated bug nets and prophylaxis medications. There currently is not enough evidence to support the notion that the risk of iron supplementation on malaria outweighs the benefits of reducing anemia. There have not yet been randomized controlled intervention trials that can prove a causal relationship between iron supplementation during pregnancy and increased risk of malaria (Sangaré et al., 2014).

Iron deficiency may also be related to a phenomenon called pica. Pica is the craving and intentional consumption of non-food items. It has been found in nearly all cultures worldwide. There are different kinds of pica, including geophagy (consumption of earth), amylophagy (consumption of raw starches) and pagophagy (consumption of ice) (Danford, 1982; Young et al., 2010). Pica can contribute to micronutrient deficiencies because the components of non-food substances can bind to nutrients, thus limiting absorption. Geophagia can also lead to intestinal parasites, which can contribute to malabsorption. Pica is most commonly found in young children and pregnant women and is usually associated with iron deficiency. In a study of Tanzanian pregnant women, $5.3 \%$ reported having geophagy during their pregnancy and 36.3\% reported amylophagy (Young et al., 2010). Common substances consumed included uncooked rice, unripe mango, large amounts of ice, and different types of clay. Women who experienced any form of pica had significantly lower hemoglobin levels compared to women without pica. In this population, every $1 \mathrm{~g} / \mathrm{dL}$ reduction in hemoglobin level correlated to a $31.5 \%$ increased likelihood of having pica. Women with later gestational age, low hemoglobin, and abdominal pain or nausea were more likely to have pica. While there is a strong 
association between pica and iron deficiency, the reasons are still not fully understood (Young et al., 2010).

Iron is the most prevalent nutrient deficiency seen in pregnant women, and the effects of prenatal iron supplementation are far-reaching. More research still needs to be done on the potentially deleterious effects of over-supplementing women who already have adequate iron stores. However, the high rates of iron deficiency in developing countries indicate that the benefits of iron supplementation (30-60 mg per day) are greater than the risks. Iron is a crucial nutrient for the proper growth and development of the fetus, as well as for the optimal health and survival of the mother.

\subsubsection{Zinc}

Zinc is a trace mineral that is necessary in the human body for many cellular processes, including protein synthesis, energy metabolism, collagen formation, and genetic expression. Dietary sources of zinc include red meats, seafood, poultry, whole grains, legumes, vegetables, and dairy products. Plant sources of zinc have lower bioavailability than animal sources (FAO \& WHO, 1998; S. S. Gropper et al., 2009).

Similar to iron, there are various ligands that can bind to zinc and influence absorption. Enhancers of zinc absorption include certain amino acids, citric acid, and products of protein digestion. Zinc absorption is regulated by zinc status; low levels of zinc will result in increased absorption. Inhibitors of zinc absorption are similar to inhibitors of iron absorption, and include phytates, oxalates, and polyphenols. Zinc binds to phytate or phytic acid with oxygen, creating a large, insoluble complex that is not well absorbed (S. S. Gropper et al., 2009). A high phytate diet is common in those who consume unrefined cereal grains with low intake of animal products (King, 2000). A 
study in Malawi found that $36 \%$ of pregnant women had low plasma zinc levels. Women who consumed high levels of phytates combined with frequent pregnancies and high rates of malaria infection were more likely to have lower levels of zinc (Gibson \& Huddle, 1998). Polyphenols are found in tannins from tea, as well as some forms of fiber found in whole grains, fruits, and vegetables. Other nutrients, including iron and calcium, may interfere with the absorption of zinc. These minerals are divalent cations like zinc $\left(\mathrm{Zn}^{2+}\right)$ and they compete for transporters and binding ligands in the intestine (Whittaker, 1998). Heme iron does not have the same effects on zinc absorption as non-heme iron does. The absorption of zinc is inhibited when the amount of non-heme iron consumed is greater than that of zinc by $20 \mathrm{mg}$. Studies on the interaction between calcium and zinc absorption vary based on type of calcium consumed (S. S. Gropper et al., 2009).

Zinc interacts with copper absorption in a negative way. High zinc intake results in increased production of thionein, a protein that is a binding ligand for zinc and becomes metallothionein when attached to a mineral. Thionein has a greater affinity for copper than it does for zinc, which traps copper in the enterocyte as part of metallothionein. This entrapment can result in copper deficiency if supplemental zinc is taken for too long or the tolerable upper intake level (UL [10 mg of elemental zinc per day]) is exceeded (Food and Nutrition Board, 2001; S. S. Gropper et al., 2009; Sandstead, 1995).

Zinc functions in many different metalloenzymes through provision of structural integrity or participation in reactions. Zinc is estimated to be involved with 70 to 200 enzymes, including those involved with liver and bone function, alcohol detoxification, respiration, protein digestion, nucleic acid synthesis, and antioxidant defense. Other 
functions of zinc include skin integrity, cell growth and replication, bone formation, and host defense immunity. Zinc is an essential component of regulating transcription and gene expression. The involvement of zinc in so many essential activities makes it crucial for proper growth, development and functioning of the body (FAO \& WHO, 1998; S. S. Gropper et al., 2009).

The RDA for zinc is set at $8 \mathrm{mg}$ per day for women, which increases to $11 \mathrm{mg}$ per day during pregnancy to account for increased needs for the fetus and placenta. Zinc status is most commonly evaluated with serum or plasma zinc, which can be affected by infection, stress, medication, and fasting status (S. S. Gropper et al., 2009). It has been estimated that $33 \%$ of the world does not consume sufficient amounts of zinc (Prado \& Dewey, 2014). The mean zinc intake of Malawian women of reproductive age is approximately two-thirds of the RDA (Huffman, Baker, Shumann, \& Zehner, 1998; Ramakrishnan, 2002). A study analyzing per capita food balance sheets and food composition tables estimated the risk of micronutrient deficiencies in many African countries. The dietary supply of zinc in Malawi was estimated to be $11.8 \mathrm{mg}$ per capita per day, while the risk of zinc deficiency was estimated to be $33 \%$ (Fig. 2.6). The mean phytate to zinc ratio in Malawi was 37.7, which is higher than any other African country (Joy et al., 2014). 

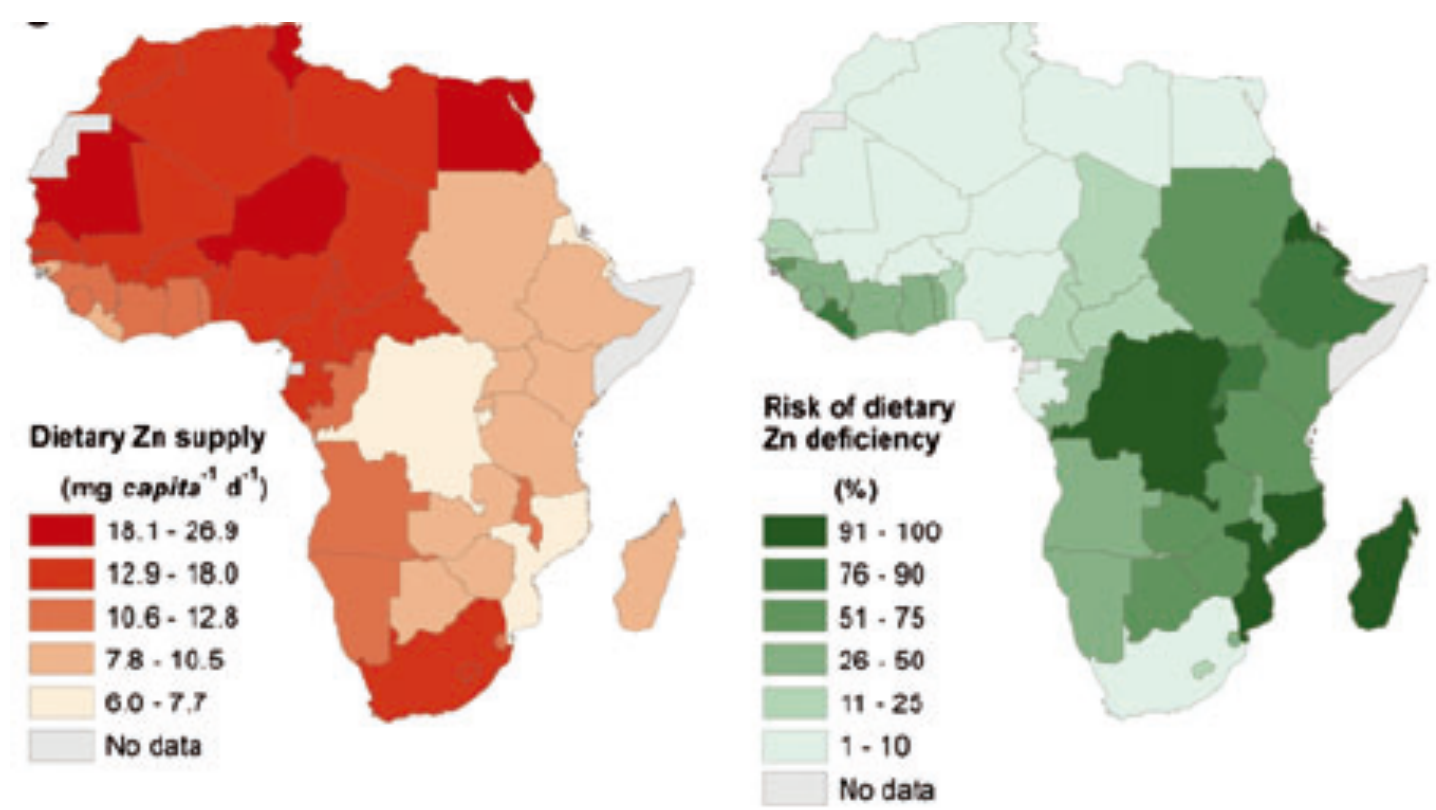

Figure 2.5. The dietary supply of zinc and the risk of zinc deficiency in African countries. Excerpted from (Joy et al., 2014).

Pregnant women and young children are at risk for zinc deficiency, due to the increased growth during these periods (FAO \& WHO, 1998). Symptoms of zinc deficiency include growth retardation, skeletal abnormalities, poor wound healing, dermatitis, delayed sexual maturation in adolescents, reduced sense of taste, hair loss and impaired immunity (S. S. Gropper et al., 2009).

Maternal zinc deficiency can cause altered DNA synthesis, chromosomal damage, oxidative stress and increased apoptosis in fetal cells, leading to abnormal embryo development (Uriu-Adams \& Keen, 2010). These factors can lead to adverse birth outcomes, including IUGR, congenital abnormalities, LBW infants, preterm delivery, and fetal death (King, 2000). Later in life, infants and children with zinc deficiency may be affected by stunted growth, abnormal cognitive development, and reduced sense of taste (hypogeusia) (Akhtar, 2013; Kennedy et al., 2003). Adverse maternal outcomes include 
pregnancy-induced hypertension, preeclampsia, hemorrhage, increased rates of infections, and prolonged labor (King, 2000).

Animal studies have shown that maternal diets devoid of zinc can cause congenital anomalies and teratogenic effects (King, 2000). Zinc may be a nutrient, like folate, that is associated with neural tube defects (NTDs). A case-control study of 61 newborns in Bangladesh found that cases with NTDs $(n=29)$ and their mothers had significantly lower serum zinc concentrations compared to control newborns $(\mathrm{n}=32)$ and their mothers. There was a significant inverse correlation (point biserial correlation $0.381, \mathrm{p}<0.05$ ) between maternal serum zinc concentration and incidence of NTDs in their infants (Figure 2.7). Women with higher parity were more likely to have a newborn with a NTD, although this was not significant. After adjustment for multiparity, newborn zinc status was still significantly correlated with incidence of NTDs, but maternal zinc status was not. In this small population, newborns with zinc deficiency were more likely to be affected by a NTD. Newborn and maternal zinc levels were highly and positively correlated $(\mathrm{r}=0.976, \mathrm{p}<0.05)$ (Dey et al., 2010).

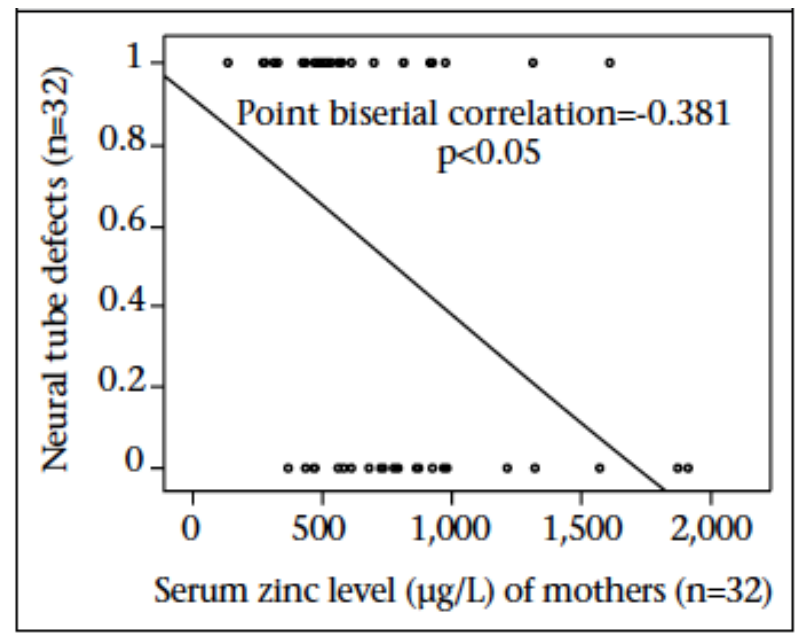

Figure 2.6. Maternal serum zinc concentration and newborn NTD incidence ( $0=$ controls; $1=$ cases with NTDs). Excerpted from (Dey et al., 2010) 
Zinc in combination with other micronutrients may be beneficial to maternal and newborn health. A randomized controlled trial in Ghana aimed to determine if combined iron and zinc supplementation beginning before 16 weeks gestation (mean 13.7 weeks) would improve iron status in pregnant women more than iron alone. Women in the iron and zinc group who were deficient in hemoglobin $(<11.0 \mathrm{~g} / \mathrm{dL})$ at baseline had a greater increase in hemoglobin $(p=0.0001)$ and ferritin concentrations $(p=0.013)$ than women in the iron-only group after an average of 18 weeks of supplementation (Saaka, 2012). In Indonesia, 2,173 pregnant women were randomized to receive zinc, vitamin A, zinc plus vitamin $\mathrm{A}$, or a placebo starting at a mean of 17 weeks gestation until delivery (Prawirohartono, Nystrom, Nurdiati, Hakimi, \& Lind, 2013). Supplementation with either zinc or vitamin A alone (not in combination) increased birth length by $0.3 \mathrm{~cm}$ and $0.2 \mathrm{~cm}$, respectively, compared to placebo $(\mathrm{p}=0.04)$. Limitations in this study include low compliance (70\%), lack of biochemical nutrient markers, unknown time of birth weight measurements, and possible low accuracy of birth length measurements (Prawirohartono et al., 2013).

Studies have shown that prenatal zinc supplementation, in addition to other nutrients, can improve maternal and newborn zinc status. A study in Peru randomized 1,295 pregnant women to receive iron and folate supplements, with or without $15 \mathrm{mg}$ zinc, beginning at 10-24 weeks gestation (L. E. Caulfield, Zavaleta, \& Figueroa, 1999). Higher zinc concentrations were found in mothers at term and newborns of the zinc group, compared to those in the iron and folate group. In this study and others (Higashi, Tajiri, Matsukura, \& Matsuda, 1988; Neggers et al., 1990; Swanson \& King, 1987), zinc 
concentrations decrease throughout pregnancy. However, the population in the study by Caulfield, et al (1999) experienced a greater decline in zinc concentrations, compared to the other studies reviewed (Figure 2.8). The authors indicate that zinc supplementation greater than $15 \mathrm{mg}$ may be necessary to improve zinc concentrations throughout pregnancy. Results from this study indicate that the addition of zinc to prenatal supplements has a beneficial effect on maternal and neonatal zinc status; thus, zinc should be considered as a standard in prenatal nutrient supplementations (L. E. Caulfield et al., 1999).

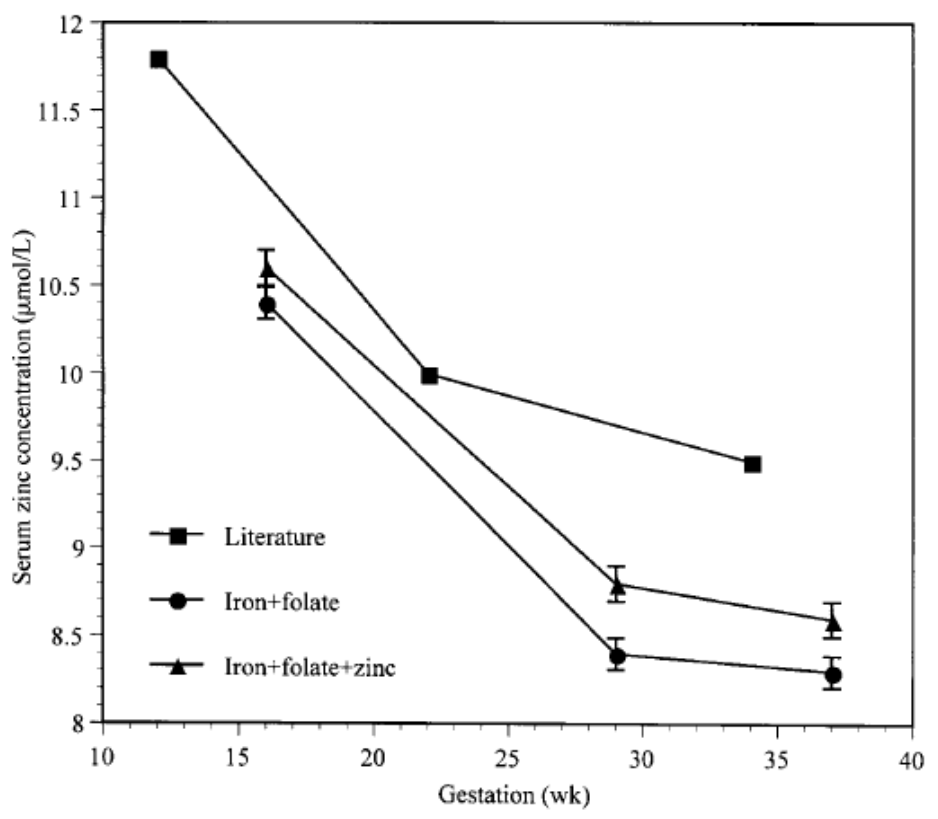

Figure 2.7. The decline of average serum zinc concentrations throughout gestation in previous literature, iron group, and iron-zinc group. Excerpted from (L. E. Caulfield et al., 1999).

Serum and plasma zinc concentrations can be affected by fasting status in nonpregnant individuals. In pregnancy, fasting status has not been found to significantly affect zinc concentrations (Hotz, Peerson, \& Brown, 2003). However, lower cut-off values for determining zinc deficiency based on trimester are proposed, due to the decline in serum zinc throughout pregnancy (Table 2.6). 
Table 2.6 Proposed lower cut-offs for assessment of zinc deficiency during pregnancy

\begin{tabular}{lc}
\hline & Lower cut-off value of serum zinc $(\mathrm{ug} / \mathrm{dL})$ \\
\hline First trimester & 56 \\
Second and third trimester & 50 \\
\end{tabular}

Adapted from (Hotz et al., 2003)

Women in developing countries may be at higher risk for zinc deficiency because of diets low in zinc and animal products, and high in factors that inhibit zinc absorption, such as phytates commonly found in cereal- and grain-based diets. The addition of supplemental zinc during pregnancy should be considered to reduce the risks associated with inadequate zinc status (King, 2000).

\subsection{Nutrition, infections, and inflammation}

The nutritional status of pregnant women can be greatly affected by the inflammatory process that occurs with infections, as well as the inflammation that occurs during pregnancy naturally. The concentrations of some micronutrients in the blood are altered by hemodilution during pregnancy, as well as by inflammation (Tomkins, 2003). This section addresses the nutrients that are affected by inflammation, including retinol, ferritin, zinc, and vitamin D, in addition to the aspects of the inflammatory response and common infections and diseases that affect Malawian women.

\subsubsection{The inflammatory response}

The inflammatory response to an acute infection, also known as the acute phase response, results in metabolic changes characterized by increased concentrations of some plasma proteins (positive acute phase proteins [APPs]) and decreased concentrations of other plasma proteins (negative APPs). C-reactive protein (CRP) is a positive APP, while retinol binding protein $(\mathrm{RBP})$, prealbumin, albumin, and transferrin are negative APPs. 
Nutrients can also be classified as positive acute phase or negative acute phase reactants. Retinol, 25-hydroxyvitamin D, and plasma zinc concentrations are reduced during an acute phase response. Ferritin is a positive acute phase reactant, which increases during the inflammatory response (S. S. Gropper et al., 2009; Thurnham \& Mccabe, 2012; Tomkins, 2003).

Inflammation is designed to be protective to the body and remove foreign invaders after a trauma or infection. Acute phase proteins protect the body by promoting wound healing and stimulating the immune system to respond to the injury (S. S. Gropper et al., 2009). However, chronic inflammation that persists for months or years due to long-term illnesses can be detrimental and will increase metabolic demands. The cascade of reactions that starts off the acute phase response typically involves macrophages, which release cytokines that trigger fibroblasts and endothelial cells to release more cytokines. When inflammatory cytokines are released, the hypothalamus responds by increasing body temperature and the liver responds by altering metabolism and stimulating production of the APPs (Thurnham \& Mccabe, 2012).

There are many APPs with different functions and properties, but they all play a role in the restoration of homeostatic balance in the body after an injury, trauma or infection (Koj, 1985; Thurnham \& Mccabe, 2012). CRP responds to stress and injury by triggering phagocytosis of white blood cells and stimulating other proteins that destroy pathogens and microorganisms with antibodies (S. S. Gropper et al., 2009). In response to inflammation, CRP will increase 20-1,000 fold in a timeframe of 24 to 48 hours and has a half-life of two days. The measurement of CRP is commonly used to assess inflammation 
levels and to correct for nutrients that are affected by inflammation. A cut-off value for CRP of $>5 \mathrm{mg} / \mathrm{L}$ is commonly used to define inflammation (Thurnham \& Mccabe, 2012).

Retinol is known to decrease during the acute phase response, but the effect that inflammation has on retinol level depends largely on the individual's overall nutritional status and the severity of the infection (Filteau \& Tomkins, 1994; Thurnham \& Mccabe, 2012; Tomkins, 2003). Plasma retinol concentrations decrease quickly in response to inflammation, while CRP rises. If inflammation is not taken into account, then prevalence of vitamin A deficiency will be overestimated (Thurnham \& Mccabe, 2012).

The inflammatory response reduces levels of circulating free iron and increases concentrations of binding and storage proteins, in order to reduce the amount of iron available to pathogens and microbes. Stress or injury will increase ferritin concentrations, even in individuals with iron deficiency. Plasma zinc is reduced during the acute phase response, which leads to decreased availability for microbial metabolism during an infection. Serum folate levels are also known to fall during an inflammatory response, while red blood cell folate is not affected (Tomkins, 2003). Many nutrients are affected by the inflammatory response; thus, it is crucial to measure concentrations of acute phase protein(s) to determine prevalence of true nutrient deficiencies.

\subsubsection{HIV/AIDS and pregnancy}

Human immunodeficiency virus (HIV) is a retrovirus that targets immune cells of the host (CD4+ T-cells) by causing them to replicate HIV. The host cell dies after viral replication is complete. Immunodeficiency occurs once the numbers of CD4+ cells are almost depleted. Secondary, or opportunistic, infections occur when CD4+ cell levels are 
low, because the body is not able to fight off infections. Yeast infections are common due to an excessive proliferation of normal bacteria (Montgomery, 2003).

Transmission of HIV occurs through person-to-person exchange of infected body fluids (sexual contact with an infected person, contaminated needles or blood transfusions, or mother-to-child-transmission [MTCT]). HIV can progress to acquired immunodeficiency syndrome (AIDS) in some, but not all, cases (Montgomery, 2003; Nelms, Sucher, Lacey, \& Roth, 2007).

There is a complex interaction between HIV, nutrition, and immunity. HIV infection affects immune function, which has a bidirectional relationship with malnutrition and secondary infections (Figure 2.9). Weight loss is usually concurrent with events of opportunistic infections. All metabolic processes are affected by HIV, which can increase protein turnover and alter carbohydrate and lipid metabolism. Hyperlipidemia is common in those infected with HIV (Macallan, 1999a). Nutrient malabsorption is increased because the majority of the body's T-cells reside in the gut (Nelms et al., 2007).

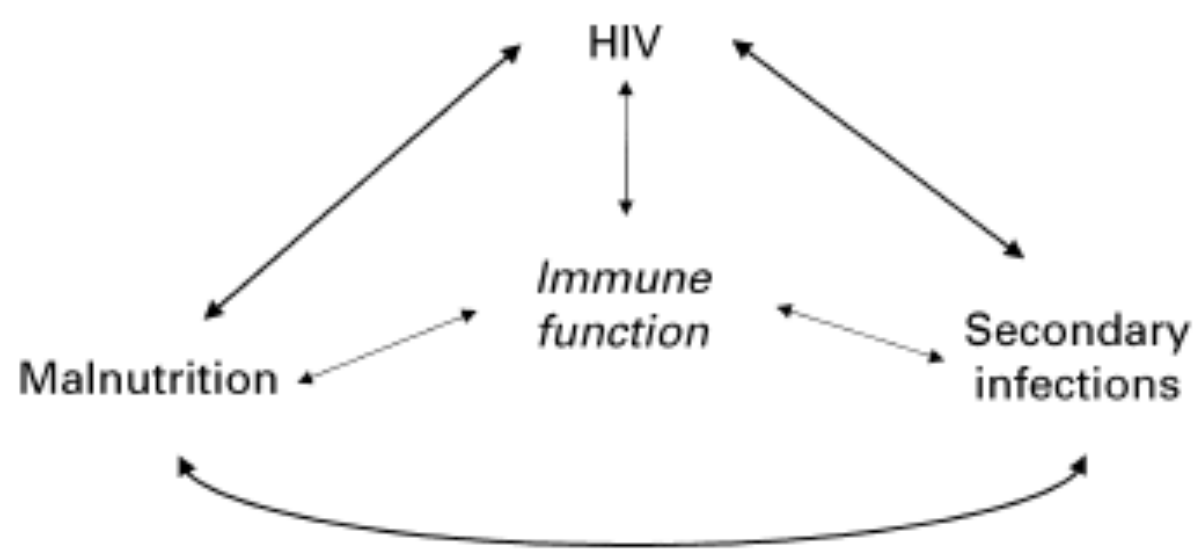

Figure 2.8. The relationships between human immunodeficiency virus (HIV), immunity, and malnutrition. Excerpted from (Macallan, 1999a). 
Individuals with HIV are living in a chronic inflammatory state, which differs intra-individually based on the severity of the infection (Nelms et al., 2007). The stressed tissues continually produce the stress hormone cortisol in response, which leads to catabolism of protein stores. The inflammatory process also affects nutrient metabolism and utilization (Fraker, 1994; Nelms et al., 2007). Protein metabolism becomes abnormal, as muscle protein stores are broken down to generate amino acids necessary to sustain the immune response (Scrimshaw \& Sangiovanni, 1997).

Wasting may occur, commonly in acute episodes of weight loss over a period of weeks to months (Macallan, 1999a). Wasting causes progressive loss of lean and fat body mass and is a strong predictor of mortality. Wasting has also been associated with higher viral load, lower CD4+ cell count, and faster disease progression (Villamor, Saathoff, Manji, et al., 2005).

Individuals with HIV tend to have altered micronutrient concentrations, which are linked to HIV disease progression. Nutrient deficiencies can lead to a worsened immune response, oxidative damage and increased susceptibility to opportunistic infections (Nelms et al., 2007). Micronutrient deficiencies are common because of increased utilization and loss, and reduced food intake and nutrient absorption (Montgomery, 2003). Important nutrients for proper immune system functioning include vitamins A, C, $\mathrm{B}_{6}, \mathrm{D}$ and $\mathrm{E}$, minerals selenium, zinc, copper, iron, magnesium and manganese, as well as beta-carotene and essential fatty acids (Nelms et al., 2007). Immunodeficiency due to inadequate micronutrient concentration has been classified as nutritionally acquired immune deficiency (NAIDS) (Beisel, 1996). NAIDS may further reduce CD4+ cell 
counts and increase susceptibility to infections, thus additionally progressing the HIV disease (Friis, 2006).

According to the WHO, 35 million people were living with HIV and 2.1 million people were newly diagnosed with HIV in 2013. Sub-Saharan Africa is the most affected area, which accounts for close to $70 \%$ of the total new HIV infections globally (WHO, 2014). Data from 2013 estimates that 500,000 women over age 15 in Malawi are living with HIV and/or AIDS (UNAIDS, 2012). A cross-sectional study of 3,825 pregnant Malawian women in their third trimester found that $30.2 \%$ were HIV-infected. Of these women, $94.3 \%$ did not know they were infected with HIV, despite almost all $(99.57 \%)$ of the women reported receiving prior antenatal care (Gay et al., 2011).

Both pregnancy and HIV infection increase caloric and nutritional needs for the mother. These needs may not be met because of reduced food intake, impaired nutrient absorption and increased nutrient losses (Friis, 2002; Montgomery, 2003).

Infection with HIV may result in adverse pregnancy or birth outcomes. A systematic review found an increased risk of infant mortality in those born to HIVinfected mothers (Brocklehurst \& French, 1998). A study in pregnant Malawian women found that those with low serum vitamin A concentrations had increased MTCT of HIV (Semba et al., 1994). However, trials of vitamin A supplementation in HIV-infected pregnant women have produced mixed results. Maternal vitamin A supplementation in Malawi decreased risk of LBW infants; however, no significant effect was found for reduction in rates of preterm delivery or MTCT of HIV (Kumwenda et al., 2002). Supplementation of vitamin A in HIV-infected pregnant South African women found reduced rates of preterm delivery, but no effect on MTCT rates (Coutsoudis et al., 1999). 
A study in Tanzania randomized 1,078 HIV-infected pregnant women to one of four treatment groups: vitamin A, multivitamin (B-complex, vitamin $\mathrm{C}$ and vitamin $\mathrm{E}$ ), multivitamin with vitamin A added, or a placebo (Fawzi et al., 2004). Women in the multivitamin groups had significantly higher CD4+ cell counts during the median followup period of 71 months, compared to women in the placebo group. Viral load was significantly reduced in women in the multivitamin group without vitamin A. Women taking multivitamins without vitamin A were less likely to die of AIDS-related causes or progress to stage 4 of the disease compared to women in the placebo group (RR 0.71, $95 \%$ CI $0.51-0.98, p=0.04)$. Women in the multivitamin plus vitamin A group saw a nonsignificant reduction in women dying of AIDS-related causes or progressing to stage 4 (RR 0.80, 95\% CI 0.58-1.10, $\mathrm{p}=0.17$ ). Multivitamins (with and without vitamin A) reduced incidence of HIV-related complications, including thrush, oral ulcers, painful tongue or mouth, and rash. Multivitamins without vitamin A resulted in a significant reduction in nausea, vomiting, diarrhea, and difficulty swallowing. Vitamin A alone did not have significant effects on any clinical outcomes. Multivitamins may enhance immunity and decrease replication of HIV, thus reducing viral load, slowing progression of the disease and reducing risk of MTCT (Fawzi et al., 2004; Friis, 2006). Micronutrient supplementation to HIV-infected pregnant women may be an intervention that could reduce complications and adverse outcomes associated with HIV and AIDS (Friis, 2006).

\subsubsection{Inflammation and anemia of chronic disease}

Individuals in a chronically inflamed state have altered iron metabolism, which can cause anemia of chronic inflammation (ACI), also known as anemia of chronic disease. ACI is typically milder than iron deficiency anemia and is characterized by 
normochromic and normocytic cells, with low reticulocyte count (indicating reduced synthesis of red blood cells) (Thurnham \& Mccabe, 2012; Weiss \& Goodnough, 2005). ACI commonly occurs in developing countries due to high frequency of infections. Common diseases that lead to ACI include malaria, HIV, cancer, and autoimmune diseases. The inflammatory response inhibits iron absorption, thus iron supplementation may not have an effect on iron status in people with ACI. ACI affects the various iron biomarkers in different ways. Serum iron and ferritin are affected by inflammation within 8 hours. Serum iron will decline by approximately $50 \%$, while ferritin increases in parallel to the rise in CRP (Thurnham \& Mccabe, 2012).

ACI is characterized by abnormal iron homeostasis, uptake, and release (Weiss \& Goodnough, 2005). In ACI, the immune response releases hepcidin, a peptide hormone that inhibits the release of iron stores, which then leads to decreased serum iron. Hepcidin is also responsible for limiting iron absorption in the small intestine. Long-term inflammation can result in altered hemoglobin and red blood cell production, as well as iron retention in storage sites, which can then lead to anemia (Petraro et al., 2013; Thurnham \& Mccabe, 2012; Weiss \& Goodnough, 2005).

\subsection{Multiple micronutrient supplementation interventions}

It is common for women of reproductive age in developing countries to have coexisting micronutrient deficiencies. For this reason, it may be necessary to provide multiple micronutrient (MMN) supplements during pregnancy (Bhutta \& Das, 2014). Micronutrients play a crucial role in the growth, development, and immune system of the fetus, as well as preventing adverse pregnancy and birth outcomes. Women who have micronutrient deficiencies during pregnancy are more likely to have infants with 
micronutrient deficiencies, thus creating an intergenerational cycle of malnutrition. Since many pregnant women in developing countries consume diets poor in essential nutrients, MMN supplements may be an appropriate method to combat micronutrient deficiencies in mothers and their infants (Haider \& Bhutta, 2012, 2015).

MMN supplements are generally cost-effective and deliver nutrients in a single delivery system. Micronutrients can be encapsulated into a pill or fortified into a supplementary food or beverage. Concerns of MMN supplementation include possible interactions between nutrients (e.g. iron and zinc) or potential overload from nutrients if consumed in excessive amounts (e.g. iron and vitamin A) (Haider \& Bhutta, 2012). Another criticism is that some women may already have adequate nutrient levels, thus making MMN supplements unnecessary or even harmful (Gopalan, 2002; Shah \& Ohlsson, 2009). Studies investigating the effectiveness of MMN supplements compared with iron and folic acid supplements alone have produced mixed results, especially in regards to maternal outcomes. Research has generally supported the hypothesis that MMN supplements result in some improved infant outcomes, such as birth weight and fetal growth, compared to iron and folic acid alone, although many of these studies are varied as well (Haider \& Bhutta, 2012; Shah \& Ohlsson, 2009).

\subsubsection{Maternal outcomes of multiple micronutrient supplementation}

Maternal micronutrient deficiency may lead to many adverse outcomes, including anemia, labor complications, hypertension, hemorrhage, and death (Ramakrishnan et al., 1999). It is hypothesized that replacing iron-folic acid supplements with MMN supplements may have a additional benefit in reducing anemia risk (Bhutta et al., 2012); however, not all studies support this hypothesis. A review of nutrition intervention trials 
found that mothers who took iron, iron and folic acid, or vitamin A supplements had significantly reduced maternal anemia risk (Imdad \& Bhutta, 2012b; Thorne-Lyman \& Fawzi, 2012); however, supplements with additional micronutrients did not further decrease anemia risk (Bhutta et al., 2012). The review found that supplements including calcium reduced risk of preeclampsia, pregnancy-induced hypertension, severe morbidity and maternal mortality (Imdad \& Bhutta, 2012a; Ramakrishnan, Imhoff-Kunsch, \& Martorell, 2014).

A randomized controlled trial in Tanzania assessed the effectiveness of a beverage fortified with 11 micronutrients on ferritin, retinol, and hemoglobin concentrations in pregnant women between 12 and 34 weeks gestation (Makola, Ash, Tatala, Latham, \& Ndossi, 2003). The intervention was eight weeks of supplementation with the fortified beverage or a placebo beverage, in addition to iron and folic acid supplementation. After the intervention period, the fortified group had a significantly lower proportion of anemic women compared to the placebo group ( $48.5 \%$ versus $37 \%$, $\mathrm{p}=0.019$ ), even though both groups received iron supplementation. Women in the fortified group were $51 \%$ less likely to have anemia post-intervention and had significantly higher ferritin and hemoglobin concentrations, compared to the women in the control group. Retinol levels were not significantly different between the groups after intervention. In this population, eight weeks of supplementation with a MMN-fortified beverage was sufficient to raise hemoglobin and ferritin levels and decrease anemia rates in pregnant women more than iron and folic acid alone (Makola et al., 2003).

A cluster trial in rural China assessed change in maternal hemoglobin concentration between three randomized groups: supplement with $15 \mathrm{MMNs}$ 
(UNIMMAP), iron and folic acid (IFA), or folic acid (FA) alone (the current antenatal standard of care in China) (Zeng et al., 2008). UNIMMAP is the international supplement prepared by the United Nations Children's Fund, World Health Organization, and the United Nations University, which contains the RDA of 15 vitamins and minerals. There was a significant increase in hemoglobin concentrations from baseline to the third trimester in women in the MMN $(+6.9 \mathrm{~g} / \mathrm{L} ; 95 \% \mathrm{CI}, 4.1-9.6 ; \mathrm{p}<0.001)$ and IFA $(+5.0$ $\mathrm{g} / \mathrm{L}, \mathrm{p}=0.001$ ) groups, compared to women in the FA group. Although over $40 \%$ of women in each group were still anemic in the third trimester, both MMN and IFA supplementation significantly increased hemoglobin concentrations (Zeng et al., 2008).

Not all studies reviewed found that MMN supplementation results in improved maternal iron status compared to IFA supplementation. In Bangladesh, a randomized controlled trial with a $2 \times 3$ factorial design aimed to determine whether timing of food supplementation in combination with micronutrient supplementation had an impact on maternal hemoglobin (Persson et al., 2012). The two food groups started either immediately after pregnancy diagnosis (“early invitation” group [average 9 weeks gestation]) or the "usual" group (average 20 weeks gestation). The food supplement contained roasted rice and pulse powders, molasses, and soybean oil, providing $608 \mathrm{kcal}$ and 18 grams of protein. The three supplement groups were IFA (30 mg iron), IFA (60 mg iron), or a MMN supplement (UNIMMAP). Hemoglobin concentrations were measured at 14 and 30 weeks gestation. The mean maternal hemoglobin at 30 weeks was $11.5 \mathrm{~g} / \mathrm{dL}$ and the prevalence of anemia was $32.9 \%$. There were no significant differences in anemia rates or hemoglobin concentrations at 30 weeks between the micronutrient supplementation groups. All three micronutrient groups experienced a small decrease in 
hemoglobin concentrations from 14 to 30 weeks gestation (Persson et al., 2012), which is consistent with the tendency of hemoglobin concentrations to decrease from first trimester to the third trimester (Scholl, 2005). The IFA group with $60 \mathrm{mg}$ iron did not have additional benefits above $30 \mathrm{mg}$ iron. In this study population with a low prevalence of iron deficiency at baseline, additional micronutrients or increased iron did not have an added effect on increasing maternal hemoglobin later in gestation (Persson et al., 2012). A study in rural China found that women who were taking prenatal MMN supplements did not have significantly different hemoglobin concentrations in the third trimester than women taking IFA supplements ( $\mathrm{p}=0.34)$ (Liu et al., 2013).

There are other nutrient biomarkers that may be affected by supplementing with additional micronutrients as opposed to iron and folic acid alone. A trial in a low-income, multi-ethnic area of East London assessed the efficacy of a MMN supplement compared to FA alone in mothers under 13 weeks gestation at baseline (Brough, Rees, Crawford, Morton, \& Dorman, 2010). Based on intention-to-treat analysis, women in the MMN group had significantly higher folate and hemoglobin concentrations at 26 and 34 weeks gestation, as well as vitamin D levels at 26 weeks, compared to women in the FA group. Although this study was affected by a high dropout rate, early MMN supplementation improved maternal nutrient status later in gestation (Brough et al., 2010).

Micronutrient supplementation may increase length of gestation, which can have beneficial effects for mother and fetus. Zimbabwean women who received a MMN supplement during pregnancy experienced an increase in gestational duration of 0.3 weeks longer than women receiving iron and folic acid, although this did not reach significance ( $\mathrm{p}=0.06$ ) (Friis et al., 2004a). A longer gestation tends to be related to 
increased birth size of infants (Friis et al., 2004a). However, a randomized controlled trial in rural China did not find a significant effect of MMN supplementation on gestational duration compared to IFA supplementation ( 0.05 weeks, CI $-0.01-0.11, \mathrm{p}=0.08)$ (Liu et al., 2013).

Among HIV-infected women, MMN supplementation improved several maternal outcomes in study in Tanzania by Fazwi, et al (1998). Women receiving a multivitamin supplement had significantly greater mean increase in CD4 cell count from baseline to 6 weeks postpartum (55.0, 95\% CI 12.51-97.49, $\mathrm{p}=0.01)$, compared to women receiving a placebo or vitamin A (in addition to the standard IFA). Multivitamins also proved beneficial to maternal anthropometry. Women who received MMN supplements saw a greater increase in weight from baseline to the third trimester, compared to women not receiving multivitamins ( $+304 \mathrm{~g}, 95 \%$ CI 17-590, $\mathrm{p}=0.04)$. Among these HIV-infected women, MMN supplements also raised hemoglobin concentrations significantly higher from baseline to 6 weeks postpartum $(+0.7 \mathrm{~g} / \mathrm{dL}, 95 \%$ CI $0.44-0.96, \mathrm{p}<0.00001)$ compared to women receiving vitamin A or a placebo (Fawzi et al., 1998).

Some research supports the hypothesis that maternal MMN supplements can reduce anemia further than IFA alone. Conflicting results of MMN supplementation on maternal outcomes indicate that more trials need to be conducted to determine the benefits of supplementing with multiple micronutrients during pregnancy.

\subsubsection{Infant outcomes of maternal multiple micronutrient supplementation}

An estimated 75 million births occur annually in developing countries (Shah \& Ohlsson, 2009), many of which result in inadequate growth or preterm delivery, which can lead to increased morbidity, mortality, stunted growth, and compromised cognitive 
development (Friis et al., 2004a). Shah and Ohlsson (2009) estimate that 1.5 million infants (95\% CI $0.75-2.25$ million) born with low birth weight $(<2500$ grams $)$ could be avoided if mothers in developing countries were given MMN supplements instead of iron and folic acid alone. Other infant outcomes that potentially could be improved from MMN supplementation include reduction in SGA infants, preterm births, neonatal and infant mortality, and stillbirths (Haider \& Bhutta, 2012; A. H. Shankar et al., 2008).

The most widely studied infant outcomes of maternal MMN supplementation assess birth size and fetal growth, including birth weight, incidence of LBW, SGA and birth length. A 2015 Cochrane Review analyzed 17 trials $(n=137,791)$ involving various interventions of maternal micronutrient supplementation. MMN supplements during pregnancy resulted in a $12 \%$ decrease in LBW infants (RR $0.88,95 \%$ CI $0.85-0.91)$ and a $10 \%$ decrease in SGA infants (RR 0.90, 95\% CI 0.83-0.97) when compared to iron and folic acid supplements alone (Haider \& Bhutta, 2015). Another meta-analysis (15 trials, $n=62,244)$ found that maternal MMN supplements increased infant birth weight by 44 grams (95\% CI 28-60 g, p=0.003) (Kawai et al., 2011).

Studies have shown a significant increase in birth weight ranging from 42 grams to 67 grams when compared to either IFA or folic acid alone, as seen in Table 2.6 (Fawzi et al., 2007; West et al., 2014; Zeng et al., 2008). A study in 1,106 Zimbabwean motherinfant pairs produced contrasting results, in which maternal MMN supplementation resulted in non-significant increases in infant birth weight of 49 grams (95\% CI; -6-104, $\mathrm{p}=0.08)$ (Friis et al., 2004a).

Infants of women receiving MMN supplements in the study by West, et al. (2014) experienced a significant mean increase in birth length of $0.20 \mathrm{~cm}$ greater than infants of 
women receiving IFA supplements during pregnancy. The studies by Zeng, et al. (2008) and Fawzi, et al. (2007) showed similar increases in birth length $(0.22 \mathrm{~cm}$ and $0.20 \mathrm{~cm}$, respectively); however, they did not reach significance due to smaller sample sizes. 
Table 2.7. Infant outcomes from trials of maternal multiple micronutrient supplementation. (Fawzi et al., 2007; A. H. Shankar et al., 2008; West et al., 2014; Zeng et al., 2008)

\begin{tabular}{|c|c|c|c|c|c|}
\hline 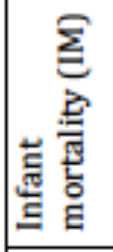 & 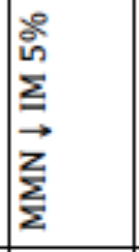 & 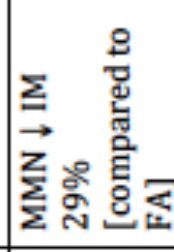 & 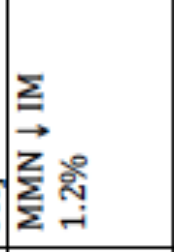 & 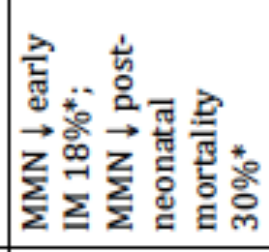 & \\
\hline 焉 & 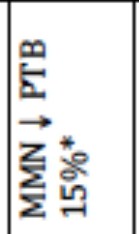 & 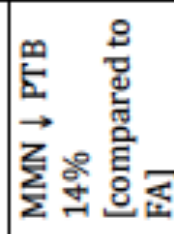 & $\mid \begin{array}{l}\infty \\
\vec{\Sigma} \\
\vec{\sum} \\
\sum \\
\Sigma\end{array}$ & 1 & \\
\hline 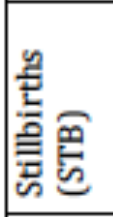 & 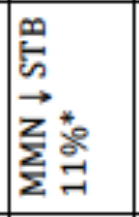 & 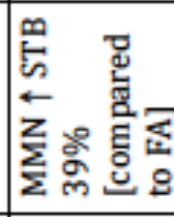 & $\begin{array}{l}\infty \\
\bar{\omega} \\
\vec{z} \\
\sum \\
\sum\end{array}$ & $\begin{array}{l}\infty \\
\sum_{\infty}^{\infty} \\
\vec{z}_{2} \\
\sum_{i}^{\circ}\end{array}$ & \\
\hline 䓌 & 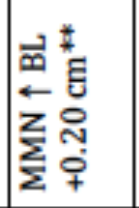 & 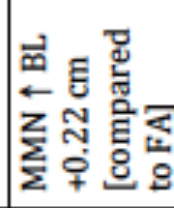 & 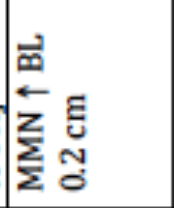 & 1 & \\
\hline 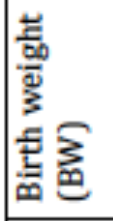 & 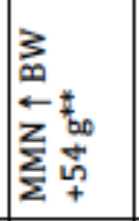 & 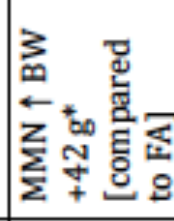 & 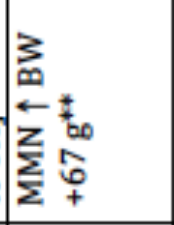 & 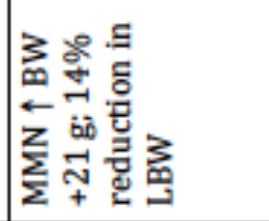 & \\
\hline 这 & 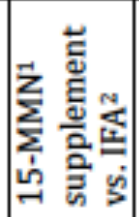 & 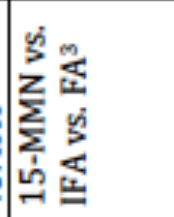 & 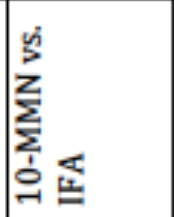 & $\sum_{\substack{\mid \\
\sum_{0}}}^{0}$ & \\
\hline 占 & 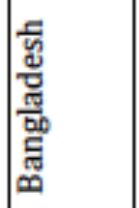 & 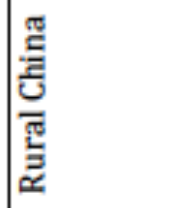 & 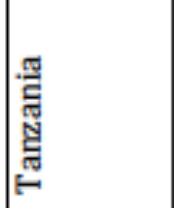 & 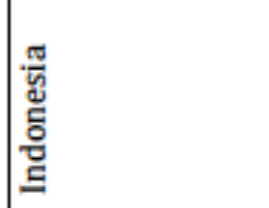 & 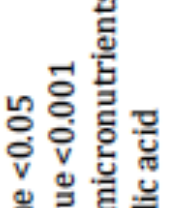 \\
\hline 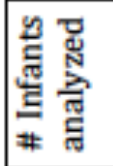 & 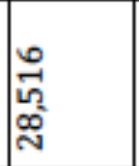 & 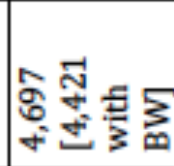 & 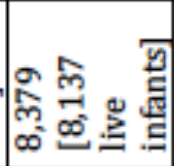 & ฟิ & 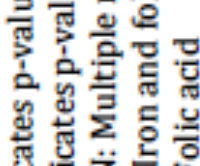 \\
\hline 依 & 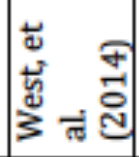 & 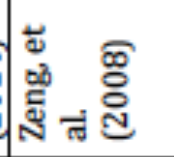 & 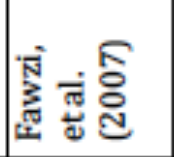 & 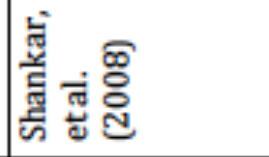 & 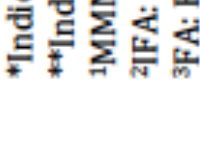 \\
\hline
\end{tabular}


Preterm births result in smaller infants, which can lead to stunted growth later in life. Bangladeshi pregnant women receiving MMN supplements experienced a 15\% reduction in preterm births compared to women receiving IFA supplements (RR 0.85, 95\% CI 0.80-0.91, $\mathrm{p}<0.001$ ) (West et al., 2014). Women in the MMN group had longer gestational durations of 0.30 weeks $(\mathrm{p}<0.001)$, which corresponded to an increase in birth weight of 55 grams (West et al., 2014). However, a Cochrane review of 15 trials of maternal supplementation in developing countries found no significant differences in preterm birth rates between MMN and IFA supplements (RR 0.96, 95\% CI 0.89-1.03) (Haider \& Bhutta, 2015). Other studies reported non-significant reductions in preterm births by $14 \%$ when comparing MMN supplements to folic acid alone (Zeng et al., 2008) and $0.7 \%$ reduction when comparing MMN supplements to IFA supplements (Fawzi et al., 2007).

Stillbirths, fetal loss, and neonatal or infant mortality are common outcomes measured in maternal supplementation trials. MMN supplements resulted in a significant reduction in stillbirths of $11 \%(\mathrm{RR} 0.89,95 \% \mathrm{CI} ; 0.81-0.99, \mathrm{p}=0.02)$ in one study (West et al., 2014), but non-significant reductions of $0.7 \%$ to $10 \%$ were found in trials from Fawzi, et al. (2007) and Shankar, et al. (2008), respectively. An interesting finding in the study by Zeng, et al. (2008) was a non-significant 39\% increase in stillbirths from women in the MMN group compared to women receiving folic acid alone (RR 1.39, 95\% CI; 0.95-2.04). Pooled data from two Nepalese trials found that MMN supplementation during pregnancy increased the risk of perinatal mortality when compared to IFA supplementation (Christian et al., 2003; Osrin et al., 2005), which can possibly be explained by the increase in fetal size. Malnourished women delivering larger babies may 
lead to difficult labors and cephalopelvic disproportions (Fawzi et al., 2007), as well as increased risk of birth asphyxia in infants who become large for gestational age (LGA) (Christian, Manandhar, Khatry, Costello, \& West, 2005). Another hypothesis is that starting supplementation early in pregnancy allows mothers to carry frail fetuses that otherwise would have spontaneously aborted, but were carried for longer gestation due to improved maternal nutritional status, resulting in perinatal death instead (Christian et al., 2005; Kawai et al., 2011).

Other studies have indicated that maternal MMN supplementation may have an impact on reducing perinatal or infant mortality. A study of 29,542 Indonesian women found an $18 \%$ reduced risk of early infant mortality (defined as death within 90 days after birth) in those taking MMN supplements $(\mathrm{p}=0.01)$ and a $30 \%$ reduced risk of postneonatal mortality (29-90 days after birth) $(\mathrm{p}=0.004)$, compared to infants of women taking IFA supplements (A. H. Shankar et al., 2008). Maternal MMN supplements also significantly reduced risk of fetal loss and neonatal mortality (defined as combined data of stillbirths, spontaneous abortions and neonatal death) by $11 \%$, compared to women taking IFA supplements (A. H. Shankar et al., 2008). As seen in Table 2.6, other studies reported non-significant reductions in infant mortality of $1.2 \%$ (Fawzi et al., 2007) and 5\% (West et al., 2014) when comparing MMN to IFA supplements, and 29\% when compared to folic acid alone (Zeng et al., 2008).

Adequate maternal micronutrient intake is crucial for fetal brain development, which has an impact on cognitive, emotional, motor, and social skills later in life. The Supplementation with Multi-Micronutrients Intervention Trial (SUMMIT) in Indonesia aimed to determine whether maternal MMN supplementation could impact the cognitive 
and socio-emotional abilities of their children at 3.5 years (Prado, Alcock, Muadz, Ullman, \& Shankar, 2012). Children of undernourished mothers (MUAC $<23.5 \mathrm{~cm}$ ) who received a supplement with 15 vitamins and minerals scored significantly higher in tests of motor development $(0.35 \mathrm{SD}, 95 \% \mathrm{CI} ; 0.01-0.69, \mathrm{p}=0.044)$ and visual attention and spatial ability ( $0.35 \mathrm{SD}, 95 \% \mathrm{CI} ; 0.08-0.63, \mathrm{p}=0.011)$ compared to children of undernourished mothers who received IFA supplements. Children born to anemic mothers receiving MMN supplements saw similar advantages in visual attention and spatial ability scores compared to those in the IFA group. No differences were found between children born to well nourished, non-anemic mothers. Cognitive abilities of children at this age are better predictors of school and work achievements in later life than infant developmental scores. This study indicates that MMN supplements may protect against the negative developmental and cognitive effects of undernutrition or anemia during pregnancy (Prado et al., 2012).

There are several potential benefits of maternal MMN supplementation to both mother and infant. However, the research is not overwhelmingly in support of changing the antenatal standard of care from IFA to MMN supplements. Birth weight is generally increased in newborns born to mothers taking MMN supplements. Other outcomes, such as maternal anemia, infant mortality, and fetal loss have produced conflicting results in maternal supplementation trials. Further research should include large randomized controlled trials to determine if there is a rationale to support MMN supplements during pregnancy. 


\subsection{Supplementary and therapeutic foods}

Ready-to-use supplementary foods (RUSF; also known as lipid-based nutrient supplements [LNS]) and ready-to-use therapeutic foods (RUTF) are increasingly being used in the treatment of acute malnutrition, especially in children and more recently, with pregnant and lactating women (Arimond et al., 2013). RUTFs are commonly provided to infants or young children with severe malnutrition and the RUTF will replace all foods other than breast milk. RUSFs, which are supplementary to the usual diet, are more typically used with children with moderate malnutrition and malnourished adults (Arimond et al., 2013). Most RUSF and RUTFs consist of powdered ingredients combined in a lipid emulsion, such as oil or peanut butter and are resistant to bacterial contamination. RUSF and RUTF are popular in developing countries because they don't require cooking or further preparation (Manary, 2005).

Fetal growth may be enhanced by maternal supplementation with protein, energy and micronutrients (Bhutta et al., 2013), however, there is a lack of controlled trials evaluating the effect of fortified energy-protein supplementary food (Ashorn, Alho, Ashorn, Cheung, Dewey, Harjunmaa, et al., 2015; Kramer \& Kakuma, 2010). A trial in Malawi assessed the impact of small-quantity lipid-based nutrient supplements (SQ-LNS) during pregnancy on birth outcomes (Ashorn, Alho, Ashorn, Cheung, Dewey, Harjunmaa, et al., 2015). Women under 20 weeks gestation were randomized to receive SQ-LNS, IFA, or MMN (containing 18 micronutrients) throughout the remainder of pregnancy $(n=1,391)$. The women were generally well nourished (mean MUAC $26.5 \mathrm{~cm}$; proportion of women with BMI $\left.<18.5 \mathrm{~kg} / \mathrm{m}^{2}: 4.6-5.9 \%\right)$. This study utilized small quantities of LNS because energy intakes were mostly adequate in this population, but 
micronutrient intakes were low. SQ-LNS provided only $118 \mathrm{kcal} /$ day but also contained micronutrients, protein, essential fatty acids, and additional minerals in a 20 -g per day dose. LNS ingredients included soybean oil, peanut paste, dried skimmed milk, vitamin and mineral mix, and sugar. Data was available from 869 infants born to women in this study. Infants from the LNS group saw a $0.2 \mathrm{~mm}(\mathrm{p}=0.006)$ and $0.1 \mathrm{~mm}(\mathrm{p}=0.175)$ increase in birth MUAC compared to the IFA and MMN groups, respectively. Infants from the LNS group had increased birth weight by $52 \mathrm{~g}$ and $36 \mathrm{~g}$ compared to the IFA and MMN groups, respectively, although these results did not reach significance. No significant differences were found between groups for SGA, preterm birth, LBW, newborn stunting, or head circumference, nor for measurements of the children at 18 months. Stillbirths were higher in the LNS group than the MMN group $(\mathrm{p}=0.006)$. Also, infants in the IFA group gained length faster (from birth to 18 months) compared to those in the LNS group. The authors hypothesize that the slower postnatal growth in the LNS infants may be due to displacement of breast milk by LNS, which could slow growth. The authors conclude that SQ-LNS did not promote increased fetal growth and birth size in this study population, although the sample size was smaller than intended (Ashorn, Alho, Ashorn, Cheung, Dewey, Harjunmaa, et al., 2015).

A trial in Burkina Faso tested the effect of fortified food supplement (FFS) against MMN supplements in pregnant women (Huybregts et al., 2009). The FFS in this study contained peanut butter, soy flour, vegetable oil, sugar and multiple micronutrients, providing $372 \mathrm{kcal}$ and $14.7 \mathrm{~g}$ protein. Pregnant women $(\mathrm{n}=1,296)$ were randomized to receive either FFS or a MMN supplement (UNIMMAP) throughout pregnancy, beginning at an average of 16 weeks gestation. The mean MUAC of women was $25.8 \mathrm{~cm}$ 
(FFS) and $26.0 \mathrm{~cm}(\mathrm{MMN}$ ), with $13.3 \%$ (FFS) and $12.5 \%$ (MMN) classified by BMI as underweight at enrollment. Birth length was $4.6 \mathrm{~mm}$ greater in the FFS group (95\% CI 1.8-7.3, $\mathrm{p}=0.001$ ) compared to the MMN group. Birth weight, stillbirths and neonatal mortality did not differ significantly between the two groups. Newborns of anemic mothers in the FFS group experienced a $143.8 \mathrm{~g}$ increase in birth weight which approached significance $(95 \% \mathrm{CI} ;-5.8-293.4, \mathrm{p}=0.059)$, compared to non-anemic mothers (Huybregts et al., 2009). An improvement in birth length can prevent stunting in early life (Schmidt et al., 2002).

A study in a semi-urban population of Ghanaian pregnant women ("iLiNS-DYAD study") was conducted to assess the impact of a LNS on fetal growth and other outcomes, when compared to MMN and IFA supplements (Adu-Afarwuah et al., 2015). The trial randomized 1,320 women to receive IFA (60 mg Fe; $400 \mu \mathrm{g}$ folic acid), MMN (20 mg Fe plus $100-200 \%$ the RDA for 18 micronutrients), or LNS (118 kcal; $20 \mathrm{mg} \mathrm{Fe;} \mathrm{same}$ micronutrients as MMN plus 4 additional minerals: $\mathrm{Ca}, \mathrm{P}, \mathrm{K}$, and $\mathrm{Mg}$ ). This study used a lower dose of iron in the MMN and LNS treatments to test the theory that a $20 \mathrm{mg}$ dose of iron is adequate during pregnancy and reduces gastrointestinal side effects, as well as because they intended to continue the treatment through lactation, which has a lower RDA for iron (9 mg/day). Infants of women in the LNS group had significantly greater mean birth weight ( $+85 \mathrm{~g})$ compared to infants of women in the IFA group, while no differences were found between the LNS and MMN groups. In this population, parity significantly affected the outcomes. Primiparous women receiving LNS had infants with significantly greater birth weight, length and head circumference compared to primiparous women receiving IFA or MMN. These authors indicate that LNS has a more 
beneficial effect on women who are nutritionally vulnerable, including those who are younger, anemic, primiparous, shorter, and of lower socioeconomic status. These demographics can also be a risk factor for fetal growth restriction, which can lead to poor infant birth outcomes. In the entire sample, LNS promoted an increase in birth weight. The macronutrients and essential fatty acids that were in the LNS treatment may promote fetal growth more than micronutrients alone (Adu-Afarwuah et al., 2015).

The iLiNS-DYAD study in Ghana also assessed maternal anemia and iron status (Adu-Afarwuah, Lartey, Okronipa, Ashorn, \& Zeilani, 2016). The women in the IFA group (60 mg iron) had greater mean hemoglobin concentrations and lower prevalence of anemia at 36 weeks gestation, compared to the MMN and LNS groups, which only had $20 \mathrm{mg}$ of iron. However, overall rates of anemia (hemoglobin $<100 \mathrm{~g} / \mathrm{L}$ ) were reduced in all groups by the end of pregnancy to $2.2-7.9 \%$, and rates of iron deficiency were $<20 \%$ across all groups. The authors conclude that $20 \mathrm{mg}$ of supplemental iron per day may not be adequate during pregnancy, but that more research is needed to determine the ideal dosage; especially because dietary iron is likely low in this population that consumes a plant-based diet, high in phytates. Furthermore, high maternal hemoglobin and iron status has been linked to adverse birth outcomes, and the study published by Adu-Afarwuah et al. (2015) mentioned above did not find that those in the IFA group had increased infant birth size. Therefore, the authors speculate whether or not there is a rationale to increase iron supplementation above $20 \mathrm{mg}$ per day, if there is in fact no impact on birth size (Adu-Afarwuah et al., 2016).

Corn-soy blend (CSB) is a common supplementary food provided to pregnant and/or lactating women in developing countries. In Cambodia, 547 pregnant women were 
randomized to receive fortified CSB flour (CSB-Plus) containing IFA and other micronutrients with oil containing vitamins A and D, or IFA alone (Janmohamed et al., 2016). The proportion of underweight women, as measured by BMI, was $37 \%$ in the CSB-Plus group and 33\% in the control group. The CSB-Plus provided $760 \mathrm{kcal}$ and $27 \mathrm{~g}$ protein per day and the oil provided an additional $90 \mathrm{kcal}$ per day. Women in the CSBPlus group had significantly lower rates of anemia at week 36-38 gestation (34\%), compared to $50 \%$ in the IFA group. There was a significantly lower rate of preterm birth in the CSB-Plus group (2.1\%) compared to the IFA group (7.1\%) $(\mathrm{p}=0.03)$. However, there were no significant differences between groups for birth weight, length, head circumference, or prevalence of LBW or SGA. An unexpected finding was no differences between groups for maternal weight gain, which was expected to increase in the CSBPlus group due to the high caloric content. The authors indicate that perhaps the women in the study shared the treatment food with other family members or that the CSB-Plus replaced her ordinary food, thus canceling out the effect of the additional calories. Women in the CSB-Plus group had significantly higher rates of fetal loss $(10.2 \%)$ compared to $3.7 \%$ in the IFA group, which is concerning. Although the CSB-Plus did not have beneficial effects on maternal or infant growth, the benefits on maternal anemia and preterm birth are worth further research (Janmohamed et al., 2016).

Other populations have benefited from RUSF products. Non-pregnant, HIVinfected adults in Malawi receiving a peanut-based RUSF experienced a greater increase in BMI and fat-free body mass compared to those receiving a corn-soy flour blend (Ndekha et al., 2009). In HIV-infected lactating women in Malawi, LNS providing 746 kcal per day resulted in an increase in B-vitamins (riboflavin, niacin, pyridoxine and 
vitamin $\mathrm{B}_{12}$ ) in breast milk compared to a control. Women receiving anti-retroviral therapy (ART) during lactation had decreased concentrations of vitamin $\mathrm{B}_{12}$, nicotinamide and pyridoxine in breast milk. In women taking LNS and ART together, the negative effects of ART were offset by the benefits of LNS increasing B-vitamins (Allen et al., 2015).

Maternal malnutrition and inadequate weight gain during pregnancy increase the risk of preterm births, LBW, and IUGR newborns. Nutritional interventions that include calories, micronutrients, protein and fatty acids may be a method to increase birth weight, reduce rates of maternal malnutrition, and improve pregnancy and birth outcomes (Yang \& Huffman, 2011). More research studies in varying populations of pregnant women, such as well nourished versus undernourished, are needed.

\subsection{Conclusions}

Maternal nutrition is an important predictor of newborn health. Micronutrient deficiencies are common in developing countries and can contribute to increased malnutrition during pregnancy, which can have adverse effects for both the mother and fetus. Women of reproductive age in developing countries are especially vulnerable to malnutrition because of increased rates of infections (HIV, malaria), poverty, food shortages and frequent pregnancies. Micronutrient deficiencies are common in areas where food diversity is low. Increased nutrient demands during pregnancy often lead to inadequate energy, protein and micronutrient intake. Malnourished pregnant women are at increased risk of morbidity and mortality, and their offspring are more likely to be born preterm or with decreased birth size. Long-term consequences of maternal malnutrition 
include stunted growth of the child, impaired immunity, and decreased cognitive, social, and educational abilities (Adair, 2014; R. Thomas et al., 2012).

Micronutrients are crucial for the proper growth and development of a fetus.

Deficiencies are common in vitamins $\mathrm{A}, \mathrm{B}_{12}$, D and folate, and minerals iron and zinc. Interventions such as multiple micronutrient supplements or RUSFs are potential methods to combat maternal malnutrition and improve birth outcomes. More research needs to be conducted, however, to determine if RUSFs are more beneficial to the mother and fetus than multiple micronutrient supplements or iron and folic acid supplements. Maternal malnutrition is a substantial issue that leads to increased morbidity and mortality of the mother and fetus, as well as adverse pregnancy and birth outcomes (Ahmed et al., 2012). 


\section{CHAPTER 3: MATERIALS AND METHODS}

\subsection{Objective}

The objective of this research was to assess micronutrient status and change in vitamin $\mathrm{A}$, vitamin $\mathrm{D}$, vitamin $\mathrm{B}_{12}$, folate, iron, zinc, albumin and $\mathrm{C}$-reactive protein concentrations in a sample of moderately malnourished pregnant Malawian women before and after receiving one of three nutritional interventions and to determine if one of those interventions improves micronutrient status better than the others.

\subsection{Subjects}

This was a sub-study ("MN sub-study") of a larger randomized controlled trial (Mamachiponde) that measured the effectiveness of the Malawian standard of care treatment for moderate malnutrition during pregnancy against two other nutritional interventions.

Pregnant women were recruited into the sub-study during enrollment into the Mamachiponde study at 15 antenatal clinics in southern Malawi between September 2014 and May 2015. The study sites were in the health districts of Blantyre, Chikhwawa, Mulanje, and Zomba.

Inclusion criteria for Mamachiponde included:

- Pregnant women with MUAC $\geq 20.6 \mathrm{~cm}$ and $\leq 23.0 \mathrm{~cm}$

- Willingness to attend the antenatal clinic every two weeks during pregnancy

- Willingness to remain in the area for delivery and until 3 months postpartum

- Provision of written and verbal consent 
- Consent to undergo HIV testing, if not done previously

Exclusion criteria for Mamachiponde included:

- Severe malnutrition (MUAC $<20.6 \mathrm{~cm}$ )

- Pregnancy complications, such as gestational diabetes, preeclampsia, or hypertension

- Severe anemia (hemoglobin $<7.0 \mathrm{~g} / \mathrm{dL}$ )

- Participation in any other nutrition study or supplemental feeding program Inclusion criteria for MN sub-study:

- Same as listed above for Mamachiponde study

- Fundal height $<22 \mathrm{~cm}$

Exclusion criteria for MN sub-study:

- Same as listed above for Mamachiponde study

- Fundal height $>22 \mathrm{~cm}$

The fundal height criterion was modified during the course of the MN sub-study. When the MN sub-study began, women with a fundal height measurement $>26 \mathrm{~cm}$ at enrollment were excluded since they would likely deliver before the second blood draw. Fundal height is a proxy for gestational age. Five months after the MN sub-study began, it was determined that the exclusion criteria should be altered to exclude women with a fundal height $>22 \mathrm{~cm}$ due to a high number of study participants delivering their child before the scheduled date of the second blood draw. At the start of both the Mamachiponde study and the MN sub-study, women below age 18 were excluded from the study. This criterion changed to include women 16 and 17 years of age two months after the micronutrient sub-study began due to the high rates of pregnant teenagers in the 
area who could benefit from the study. As a token of appreciation, the mother was provided with soap and plates after the first blood draw and a chitenje (cloth) after the second blood draw.

This study is registered in ClinicalTrials.gov NCT02120599 and was approved by the Institutional Review Boards at Washington University (St. Louis), Cal Poly (San Luis Obispo), and College of Medicine at University of Malawi.

\subsection{Randomization and blinding}

Subjects were randomized in blocks of 60 to receive one of the three nutritional treatments (RUSF, CSB-UNIMMAP, or CSB-IFA) using a random number generator, which assigned participants to a study group labeled A, B, or C. Pregnancy ID's were placed individually in blank envelopes and each participant picked out one envelope containing her treatment assignment. The research team was blinded to treatment group for each participant; each mother was provided with a large opaque study bucket so that researchers could not see the visibly different treatment food that the mothers took home.. The study nurses and drivers were the only study personnel who were aware of which treatment group the participants were in. The study nurses needed to know treatment assignment in order to give each participant the correct instructions of how to prepare and/or take the foods and supplements. After the study participants completed all enrollment information and measurements in the clinic, she would go out to the study vehicle to collect her food The study driver would look up her study number to identify which treatment she would receive, and placed it into a colored opaque bucket so that neither research team members nor other study participants could see which treatment group the woman was on. Study participants were not blinded to which treatment group 
they were in, because the RUSF is visually distinct from the CSB flour and the UNIMMAP supplement is visually distinct from the iron and folic acid supplements. Data entry and data analysis team members were blinded to group assignment.

\subsection{Study design}

Mamachiponde is a researcher-blinded, randomized controlled trial designed to determine whether provision of a micronutrient-fortified ready-to-use supplementary food (RUSF) or a fortified corn soy blended flour (CSB+) plus a multiple micronutrient supplement provided to pregnant women with moderate malnutrition will result in improved recovery from malnutrition and improved maternal and infant outcomes, compared to women receiving the standard of care. The standard of care is fortified CSB flour plus daily iron and folic acid supplements. All treatment foods and supplements were provided for a 14-day supply.

The original sample size was projected to be 300 women total ( 3 intervention groups, 100 women per group), to detect at least a 0.5 standard deviation unit difference between groups for all nutrients, with 0.05 level of significance and $80 \%$ power. The sample size was increased during the study to account for higher rates of dropouts than anticipated. A total of 343 women were enrolled in this study and had the first blood draw performed, with 335 of the women having analyses completed of their baseline nutrient levels. Eight women did not have their serum analyzed, due to reasons including hemolyzed samples, inadequate amount of blood drawn, and samples being lost. A total of 229 women completed the study with both blood draws being performed.

The three intervention groups are described in detail below. Full nutrient profiles are provided in Appendix B. 
1) RUSF: This treatment provided $900 \mathrm{kcal} / \mathrm{day}, 38 \mathrm{~g}$ protein/day, and $200 \%$ of the Recommended Dietary Allowance (RDA) for most micronutrients during pregnancy (except for vitamin $\mathrm{A}$, vitamin $\mathrm{B}_{3}$, folic acid, iodine, magnesium, and calcium, which will remain near $100 \%$ ). The energy content of RUSF was designed to provide $360-450$ calories required during the second and third trimesters of pregnancy, and an additional 450-550 calories to support recovery from moderate malnutrition. Women randomized to this treatment arm did not receive any additional supplements since micronutrients were already in the formulated food.

2) CSB + UNIMMAP: This treatment included 5 kg every two weeks of a cornsoy blended $(\mathrm{CSB}+)$ flour plus a standard antenatal micronutrient tablet (UNIMMAP) that contains 15 micronutrients, which together provided a fairly similar amount of energy, protein, and micronutrients as the RUSF treatment.

3) CSB-IFA: This is the control group, which is the Malawian standard of care treatment for moderate and severe malnutrition during pregnancy. This treatment included $5 \mathrm{~kg}$ CSB every two weeks plus daily iron $(60 \mathrm{mg})$ and folic acid (400 $\mathrm{mcg})$ supplements.

Enrolled women visited the clinic every 2 weeks for measurements, health checks and received their two-week supply of treatment food. Women who achieved a MUAC $>23.1 \mathrm{~cm}$ were considered 'graduated'. Women whose MUAC remained $>23.1 \mathrm{~cm}$ for two consecutive visits no longer received food treatment, however, she still received iron and folic acid supplements. After graduation, women came to clinic every month to ensure no relapse into malnutrition had occurred. If relapse (MUAC <23.1) occurred, mothers went back onto their assigned treatment food/supplements. Women who 
graduated and/or relapsed were still included in the analysis. Blood draws were taken at two time points, enrollment into the study and ten weeks later (at visit five), as described in detail in Section 3.5.

\subsection{Data collection}

Women eligible and willing to consent in the primary study and sub-study had anthropometrics measured by a study team member. Anthropometrics included height, weight, MUAC, triceps skin fold (TSF), and blood pressure (BP Omron Intellisense BP760). Weight was measured in kilograms using a Detecto Slimpro scale (Webb City, MO). Height was measured in centimeters using a Seca stadiometer (Birmingham, United Kingdom). MUAC was measured twice with a TALC measuring tape (Herts, UK) and if the measurements were $>1 \mathrm{~mm}$ different from each other, a third measurement was made and the two closest measurements were recorded; TSF was measured twice using Lange skinfold calipers (Beta Technology, Santa Cruz, CA) and similarly to MUAC, if the measurement differed by $>1 \mathrm{~mm}$, an additional measurement was taken and the two closest measurements were recorded. Hemoglobin was tested using Hemocue Hb 201+ (Angelholm, Sweden). Fundal height was measured by Malawian nurses and recorded to the nearest $0.5 \mathrm{~cm}$ by a standard measuring tape. Trained Malawian staff interviewed subjects and recorded information regarding demographics, clinical symptoms, household food security and food consumption frequency.

If the subject was determined to be eligible for the sub-study, she was invited to participate in the micronutrient sub-study. A study nurse reviewed the sub-study information in Chichewa, the primary language in Malawi, and if she consented to participate, the subject either signed her name (if able to write) or placed her thumb into 
an inkpad and signed with a thumbprint. The "Mamaponde Micronutrient Sub-study

Form" (see Appendix C) was filled out by a study team member. It included her

Mamachiponde study ID number, barcode ID number for specimen tubes, fundal height, hemoglobin, time of blood draw, and fasting status. If she was not fasting, it was recorded how long ago she ate (in hours).

\subsection{Sample collection}

Blood samples were taken at two separate occasions from each eligible and willing subject. The first blood draw took place when the subject enrolled into the Mamachiponde study and the second blood draw took place 10 weeks later. Venous blood samples were drawn in the morning with 21 gauge $x$ 1" blood collection needles (Jelco; Smiths Medical) using Universal Precautions into serum separator tubes ("Tiger Top" BD Vacutainer tubes; tube size of 16x100 mm; draw volume capacity of $7.5 \mathrm{~mL}$ ) for vitamins $\mathrm{B}_{12}, \mathrm{~A}, \mathrm{D}$, folate and proteins albumin and C-reactive protein (CRP), and into Trace Element heparin tubes for the mineral zinc (dark blue/royal blue BD Vacutainer; tube size of $13 \times 100 \mathrm{~mm}$; draw volume capacity of $6.0 \mathrm{~mL}$ ). The amount of blood that was drawn from each subject was approximately 9-10 $\mathrm{mL}$ total $(3-4 \mathrm{~mL}$ into the Trace Element Vacutainer and $6 \mathrm{~mL}$ into the Serum Separator Tubes). This amount of whole blood allowed for 4-5 mL of serum to be separated and used for analysis. The procedures can be found in Appendix D.

After a study nurse drew the blood, tubes were labeled with a barcode number corresponding to the subject's study ID number, covered with foil to protect from light, and packed in a small cooler box with ice packs for transport. The tubes were centrifuged at $3500 \mathrm{rpm}$ for 10 minutes at $<25^{\circ} \mathrm{C}$ (Eppendorf Centrifuge 5804R) and the serum was 
pipetted off into separate storage tubes (Rainin Classic Air Displacement Pipette PR-100; Mettler Toledo; Oakland, CA) at the College of Medicine in Blantyre, Malawi. The time of centrifugation was recorded to ensure that the time between blood draw and centrifugation was less than 4 hours. Two $\mathrm{mL}$ of serum were pipetted into two separate amber-colored storage tubes (to block out light) for analysis of vitamins $\mathrm{B}_{12}, \mathrm{~A}, \mathrm{D}$, folate, ferritin, and proteins albumin and CRP, and $1 \mathrm{~mL}$ of serum was pipetted into a clear storage tube for zinc analysis. These tubes were stored at $-80^{\circ} \mathrm{C}$ in freezers at the College of Medicine (Thermo-Scientific $-80^{\circ} \mathrm{C}$ Revco Elite Plus Freezer). The samples were shipped back to the United States periodically in nitrogen gas tanks for analysis at Central Coast Pathology (CCP) laboratory in San Luis Obispo, CA. One amber storage tube was used to analyze ferritin, vitamin $\mathrm{B}_{12}$, folate, albumin, and $\mathrm{CRP}$ at CCP laboratory. The second amber storage tube was sent to Physician's Automated Laboratory (PAL) in Bakersfield, CA for vitamins A and D analysis. The clear storage tube was sent to Clinical Pathology Lab (CPL) in Texas for zinc analysis.

The minimum serum requirements are as follows:

- $0.5 \mathrm{~mL}$ serum for vitamin $\mathrm{B}_{12}$, folate, ferritin, and retinol

- $0.3 \mathrm{~mL}$ serum for vitamin $\mathrm{D}$

- $0.2 \mathrm{~mL}$ serum for albumin

- $0.3 \mathrm{~mL}$ for CRP)

- $0.5 \mathrm{~mL}$ serum for zinc 


\subsection{Nutrient assay methods}

Table 3.1 Assays used for analysis of nutrients and proteins

\begin{tabular}{lll}
\hline Nutrient/protein & Assay method-CCP & Assay method-PAL ${ }^{2}$ \\
\hline Retinol & Nephelometry & Chemiluminescence \\
25-hydroxyvitamin D & N/A & $\begin{array}{l}\text { Chemiluminescence } \\
\text { (Liason XL Diasorin) }\end{array}$ \\
Ferritin & Electrochemiluminescence & $\begin{array}{l}\text { Chemiluminescence } \\
\text { (Beckman Coulter DXI) }\end{array}$ \\
Zinc & $\begin{array}{l}\text { Immunoassay (ECLIA) } \\
\text { Quantitative Inductively Coupled }\end{array}$ & Plasma-Mass Spectrometry \\
Folate & Plasma-Mass Spectrometry & \\
Vitamin B & ECLIA & N/A \\
& ECLIA & Chemiluminescence \\
Albumin & & (Beckman Coulter DXI) \\
& Bromcresol Green (BCG) & BCG (Beckman Coulter \\
C-reactive protein & Immunoturbidimetric & DXI) \\
& & Immunoturbidimetric \\
& & (Beckman Coulter 5800) \\
\hline
\end{tabular}

${ }^{1}$ Central Coast Pathology; ${ }^{2}$ Physician's Automated Laboratory

Table 3.2 Normal deficiency and marginal status cut-off values for pregnancy and deficiency values in inflammatory state

\begin{tabular}{|c|c|c|c|c|c|}
\hline Nutrient & Units & $\begin{array}{l}\text { Severely } \\
\text { deficient }\end{array}$ & Deficiency & $\begin{array}{c}\text { Deficiency } \\
\text { with } \\
\text { inflammation }\end{array}$ & $\begin{array}{c}\text { Marginal cut- } \\
\text { off values }\end{array}$ \\
\hline Folate $^{1}$ & $\mathrm{ng} / \mathrm{mL}$ & & $<3.0$ & -- & $3.0-5.9^{1}$ \\
\hline Vitamin $\mathrm{B}_{12}^{2}$ & $\mathrm{pg} / \mathrm{mL}$ & & $<203.0$ & -- & -- \\
\hline Vitamin $\mathrm{D}^{3}$ & $\mathrm{ng} / \mathrm{mL}$ & $<12.0$ & $12-20$ & -- & $20-30$ \\
\hline Ferritin & $\mathrm{ng} / \mathrm{mL}$ & & $<15.0$ & $<19.0^{4}$ & -- \\
\hline Retinol & umol/L & & $<0.70$ & $<0.60^{4}$ & $<1.05$ \\
\hline Albumin & $\mathrm{g} / \mathrm{dL}$ & & $<3.4$ & -- & -- \\
\hline Zinc & $\mathrm{ug} / \mathrm{dL}$ & & $\begin{array}{l}1^{\text {st }} \text { trimester }<57^{5} \\
2^{\text {nd }} / 3^{\text {rd }} \text { trimester: } \\
<50\end{array}$ & -- & -- \\
\hline CRP & $\mathrm{mg} / \mathrm{L}$ & & Elevated $>5.0$ & -- & -- \\
\hline
\end{tabular}

${ }^{1}$ Cut-off values determined from (WHO, 2012c)

${ }^{2}$ Cut-off value determined from (WHO, 2008)

${ }^{3}$ Vitamin D cut-off values determined using ARUP Laboratory Reference Ranges

${ }^{4}$ Inflammation cut-off values determined from (Thurnham \& Mccabe, 2012) 
${ }^{5}$ Zinc deficiency cut-off values for pregnancy determined from (Abbassi-Ghanavati, Greer, \& Cunninghahm, 2009; Hotz et al., 2003)

Inflammation cut-off values were used to determine prevalence of deficiency (\%) when using chi-square testing for categorical variables. CRP was used to control for the acute phase response when using ANOVA models for continuous response variables. During pregnancy, different zinc cut-off values were used for each trimester to control for the inflammatory process (Table 3.2) (Abbassi-Ghanavati et al., 2009).

\subsection{Statistical analysis methods}

Baseline characteristics and differences between mothers were tested for significance using the chi-squared test for categorical variables and the Student's t-test for continuous variables. For longitudinal measures, the groups were compared using repeated measures mixed model analysis of variance (MANOVA) to analyze mean change in nutrients from week 0 to week 10, controlling for the inflammatory response with CRP. The mixed model analysis also tested for any interactions between treatment group and time. Percent deficiency rates were compared using chi-square testing, while controlling for CRP. Nutrient concentrations at week 10 were analyzed by analysis of variance (ANOVA) and Tukey's HSD test to determine if any treatment groups were different from one another. Covariates included in this model were nutrient concentration at week 0, baseline CRP, CRP at week 10, parity, HIV status, and maternal baseline MUAC.

Normality was assessed by analyzing histograms of residuals and goodness-of-fit tests. To achieve normality, vitamin $\mathrm{B}_{12}$ and ferritin were transformed logarithmically and albumin achieved normality using square root transformation. The p-values were obtained using the transformed data while the means were displayed from the raw data. 
Outliers were removed from vitamin $\mathrm{B}_{12}(\mathrm{n}=2)$, ferritin $(\mathrm{n}=2)$, and zinc $(\mathrm{n}=1)$ to achieve a normal distribution. 


\section{CHAPTER 4: RESULTS}

\subsection{Enrollment characteristics}

A total of 343 women were enrolled into this study. Some samples from both blood draws were not included due to physical loss, hemolysis of blood, or inadequate amount of serum to analyze (Figure 4.1). The primary reason of subjects not completing the study was due to delivery before the second blood draw date $(n=62)$. Other reasons included refusal $(n=7)$, miscarriage $(n=1)$, infant death $(n=1)$, and loss to follow up $(n=7)$.

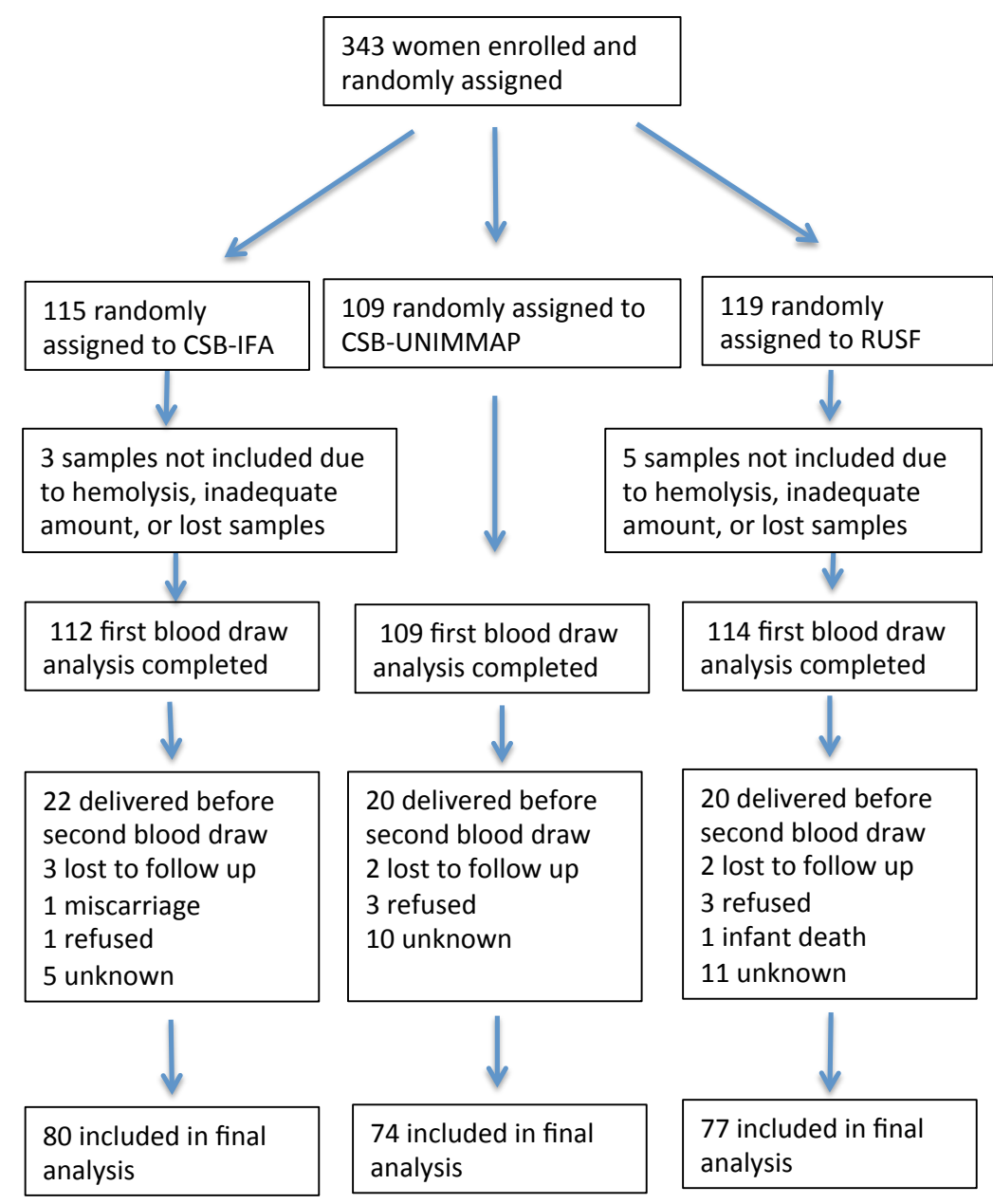

\section{Figure 4.1 Flow chart of enrollment into the study}




\subsection{Baseline characteristics}

The age range of participants was 16 to 38 years and the median age was 19 years. Educational level ranged from no schooling $(n=27)$ to tertiary school $(n=3)$, with the most common educational level as four to six years $(n=135)$. The number of other adults in the household ranged from one to eight. The RUSF group had significantly more adults in the household at baseline than CSB-UNIMMAP or CSB-IFA $(p=0.029)$ (Table 4.1). Twenty-one percent of participants experienced one or more illness within the two months before enrollment, including diarrhea (6\%), pneumonia (3\%), and malaria (15\%). All groups had similar proportions of women reporting malarial and diarrheal illness, however, pneumonia prevalence was significantly different between groups, with the CSB-IFA group having the most women with pneumonia in the last two months (Table 4.1).

The average weeks of gestation at baseline differed significantly by treatment group. CSB-UNIMMAP and CSB-IFA had greater gestational ages at enrollment (19.64 and 19.57 weeks, respectively), compared to the RUSF group (18.42 weeks) $(p=0.04)$ (Table 4.1). Parity ranged from 0 to 9 previous pregnancies, with a mean of 0.9 previous pregnancies. Fifty-four percent of women were primiparous and $46 \%$ were multiparous. The majority of the participants were already taking supplements at study enrollment (folic acid and/or iron), which is consistent with Malawian antenatal care guidelines, which would be stopped once she started participating in this study. Mean baseline height $(154.1 \mathrm{~cm})$, weight $(45.8 \mathrm{~kg})$, MUAC $(22.2 \mathrm{~cm})$, and BMI $\left(19.4 \mathrm{~kg} / \mathrm{m}^{2}\right)$ did not differ significantly by treatment group. The proportion of HIV-positive women at enrollment was greater in the CSB-IFA group (15.6\%), compared to CSB-UNIMMAP (9.2\%) and 
RUSF (8.7\%), although this was not significant. Although the overall average of women with HIV was $11.2 \%(n=38)$, only 18 women reported taking anti-retroviral therapy (ARV) (Table 4.1). HIV status was significantly related to number of previous pregnancies. Women with lower parity were more likely to be HIV-positive $(p=0.001)$. Primiparous women were 4.1 times more likely to be HIV-positive, compared to multiparous women (95\% CI for OR: 1.4, 12.1, $\mathrm{p}=0.008)$. Age at enrollment approached a significant relationship with HIV status. Older women were slightly more likely to be HIV-positive; the mean age of HIV-positive women was 23.2 years and the mean age of HIV-negative women was $20.3(\mathrm{p}<0.001)$. When age was used as a categorical variable, $21 \%$ of HIV-infected women were $16-17$ years, $37 \%$ were $18-21$ years, $16 \%$ were $22-29$ years, and $26 \%$ were $30-40$ years $(\mathrm{p}=<0.0001)$.

Table 4.1 Baseline characteristics of the participants at enrollment, by treatment group

\begin{tabular}{|c|c|c|c|c|}
\hline Baseline characteristic & $\begin{array}{l}\text { RUSF } \\
(n=119)\end{array}$ & $\begin{array}{l}\text { CSB- } \\
\text { UNIMMAP } \\
(n=109)\end{array}$ & $\begin{array}{l}\text { CSB-IFA } \\
(n=115)\end{array}$ & P-value ${ }^{1}$ \\
\hline Age at enrollment & $20.2 \pm 4.2^{2}$ & $20.7 \pm 0.9$ & $21.0 \pm 4.8$ & 0.38 \\
\hline $16-17 y(\%)$ & 25.6 & 13.6 & 19.0 & 0.106 \\
\hline $18-21 y$ & 44.4 & 59.1 & 53.4 & \\
\hline $22-29 y$ & 23.1 & 23.6 & 18.1 & \\
\hline $30-40 y$ & 6.8 & 3.6 & 9.5 & \\
\hline \multicolumn{5}{|l|}{ Educational level } \\
\hline None $(\%)$ & 6.7 & 11.0 & 6.1 & 0.48 \\
\hline $1-3 y$ & 15.1 & 17.4 & 19.1 & \\
\hline $4-6 y$ & 37.0 & 36.7 & 45.2 & \\
\hline $7-8 y$ & 26.9 & 22.9 & 13.9 & \\
\hline Secondary & 13.4 & 11.0 & 14.8 & \\
\hline Tertiary & 0.8 & 0.9 & 0.9 & \\
\hline Number of children in household & $0.7 \pm 1.2$ & $0.8 \pm 1.1$ & $0.9 \pm 0.2$ & 0.46 \\
\hline Number of adults in household & $2.4 \pm 1.1$ & $2.2 \pm 0.6$ & $2.2 \pm 0.7$ & $0.03 *$ \\
\hline Illness in the last 2 months ( $\%)$ & 19.3 & 21.1 & 22.6 & 0.18 \\
\hline Diarrhea in the last 2 months $(\%)$ & 6.0 & 6.4 & 4.3 & 0.77 \\
\hline
\end{tabular}




\begin{tabular}{|c|c|c|c|c|}
\hline $\begin{array}{l}\text { Pneumonia in the last } 2 \text { months } \\
(\%)\end{array}$ & 0.0 & 3.6 & 5.2 & $0.01 *$ \\
\hline Malaria in the last 2 months (\%) & 16.2 & 13.6 & 15.5 & 0.85 \\
\hline Uses a bed net (\%) & 76.5 & 73.4 & 69.6 & 0.49 \\
\hline Currently uses tobacco (\%) & 0 & 1.8 & 1.7 & 0.34 \\
\hline Weeks gestation at enrollment & $18.4 \pm 4.4$ & $19.6 \pm 4.0$ & $19.6 \pm 3.8$ & $0.04 *$ \\
\hline Number of previous pregnancies & $0.9 \pm 1.6$ & $1.0 \pm 1.3$ & $1.0 \pm 1.3$ & 0.86 \\
\hline Number of live births & $0.7 \pm 1.3$ & $0.8 \pm 1.3$ & $0.9 \pm 1.2$ & 0.34 \\
\hline Number of miscarriages & $0.1 \pm 0.3$ & $0.04 \pm 0.2$ & $0.08 \pm 0.3$ & 0.44 \\
\hline Number of stillborn pregnancies & $0.08 \pm 0.34$ & $0.09 \pm 0.3$ & $0.02 \pm 0.1$ & 0.10 \\
\hline $\begin{array}{l}\text { Currently taking medicine } \\
\text { Amoxicillin (\%) } \\
\text { ARV } \\
\text { Malarial treatment } \\
\text { Paracetamol } \\
\text { Other }\end{array}$ & $\begin{array}{l}24.4 \\
0 \\
4.2 \\
9.2 \\
12.6 \\
5.9\end{array}$ & $\begin{array}{l}22.0 \\
0.9 \\
2.8 \\
8.3 \\
12.8 \\
4.2\end{array}$ & $\begin{array}{l}30.4 \\
0.9 \\
9.2 \\
7.8 \\
13.0 \\
7.0\end{array}$ & 0.34 \\
\hline $\begin{array}{l}\text { Currently taking supplements } \\
\text { Folic acid (\%) } \\
\text { Iron (\%) }\end{array}$ & $\begin{array}{l}72.3 \\
2.5 \\
69.7\end{array}$ & $\begin{array}{l}70.6 \\
0.9 \\
68.8\end{array}$ & $\begin{array}{l}67.8 \\
3.5 \\
64.4\end{array}$ & 0.76 \\
\hline $\begin{array}{l}\text { Roof material of home } \\
\text { Metal sheets (\%) } \\
\text { Thatch } \\
\text { Other } \\
\text { Food insecure household }(\%)\end{array}$ & $\begin{array}{l}21.0 \\
78.2 \\
0.8 \\
28.6\end{array}$ & $\begin{array}{l}20.2 \\
79.8 \\
0 \\
36.7\end{array}$ & $\begin{array}{l}15.6 \\
84.3 \\
0 \\
30.4\end{array}$ & 0.49 \\
\hline HIV status (\% positive) & 8.7 & 9.2 & 15.6 & 0.18 \\
\hline Baseline height (cm) & $154.3 \pm 5.3$ & $153.9 \pm 5.4$ & $154.1 \pm 5.4$ & 0.91 \\
\hline Baseline MUAC (cm) & $22.2 \pm 0.6$ & $22.2 \pm 0.6$ & $22.2 \pm 0.6$ & 0.83 \\
\hline Baseline weight (kg) & $45.9 \pm 3.8$ & $45.8 \pm 3.8$ & $45.7 \pm 3.4$ & 0.93 \\
\hline Baseline BMI $\left(\mathrm{kg} / \mathrm{m}^{2}\right)$ & $19.3 \pm 1.4$ & $19.3 \pm 1.3$ & $19.5 \pm 3.0$ & 0.740 \\
\hline Baseline underweight $(\%)^{4}$ & 28.7 & 25.0 & 28.3 & 0.795 \\
\hline
\end{tabular}

\footnotetext{
${ }^{1} \mathrm{P}$-values were obtained by ANOVA for continuous variables or chi-squared test for proportions

${ }^{2}$ Mean \pm SD (for all such values unless noted otherwise)

${ }^{3} \mathrm{ARV}$ : anti-retroviral therapy

${ }^{4}$ Underweight determined by BMI $<18.5 \mathrm{~kg} / \mathrm{m}^{2}$

$*$ Indicates a significant p-value $(<0.05)$
} 


\subsection{Laboratory results}

Baseline laboratory results indicate moderate to high levels of deficiency in multiple nutrients (Table 4.2). No significant differences were found between treatment groups in deficiency rates or baseline mean concentrations. The majority of participants were deficient in hemoglobin at baseline (67.5-75.2\%); however, lower proportions were deficient in ferritin (22.3-23.9\%). Unadjusted ferritin concentrations at baseline were greater in the RUSF group (61.8 ng/mL), compared to CSB-UNIMMAP $(51.7 \mathrm{ng} / \mathrm{mL})$ and CSB-IFA (45.1 ng/mL), although this did not reach significance $(\mathrm{p}=0.09)$.

Nutrients of concern with high rates of deficiency included ferritin $(22.9 \%)$, and zinc $(35.4 \%)$, as well as marginal vitamin D (31.9\%), retinol $(32.4 \%)$ and folate $(22.4 \%)$ concentrations. Low serum albumin was found in $42.7 \%$ of participants and $50.7 \%$ had elevated C-reactive protein (CRP) (Table 4.2). These deficiency rates were adjusted for inflammation (via CRP) for ferritin, retinol and albumin and adjusted for the effect of trimester for zinc and CRP (Table 4.2). Vitamin D was classified into multiple categories. None were severely deficient $(<12 \mathrm{ng} / \mathrm{mL})$ at baseline, $3.2 \%$ were deficient $(<20 \mathrm{ng} / \mathrm{mL})$, $31.9 \%$ had marginal status $(20-30 \mathrm{ng} / \mathrm{mL})$, and $64.8 \%$ were sufficient in vitamin $\mathrm{D}$.

Table 4.2. Unadjusted serum nutrient/protein means and adjusted proportions of deficiency at baseline

\begin{tabular}{|c|c|c|c|c|}
\hline Nutrient/protein & $\begin{array}{l}\text { CSB-IFA } \\
(n=112)\end{array}$ & $\begin{array}{l}\text { CSB- } \\
\text { UNIMMAP } \\
(n=109)\end{array}$ & $\begin{array}{l}\text { RUSF } \\
(n=114)\end{array}$ & $\begin{array}{l}P- \\
\text { value }^{1}\end{array}$ \\
\hline Capillary hemoglobin (g/dL) & $10.0 \pm 1.5^{2}$ & $9.9 \pm 1.5$ & $10.3 \pm 1.6$ & 0.27 \\
\hline$\%$ deficient ${ }^{3}$ & 71.6 & 75.2 & 67.5 & 0.44 \\
\hline Serum folate $(\mathrm{ng} / \mathrm{mL})$ & $9.2 \pm 4.3$ & $10.1 \pm 4.9$ & $9.9 \pm 4.6$ & 0.25 \\
\hline$\%$ marginal $^{4}$ & 27.7 & 20.2 & 19.3 & 0.25 \\
\hline Serum vitamin $B_{12}(\mathrm{pg} / \mathrm{mL})$ & $309.2 \pm 148.7$ & $308.3 \pm 143.0$ & $313.7 \pm 145.2$ & 0.96 \\
\hline$\%$ deficient $^{5}$ & 17.9 & 22.0 & 23.7 & 0.55 \\
\hline Serum vitamin $\mathrm{D}(\mathrm{ng} / \mathrm{mL})$ & $33.2 \pm 7.7$ & $34.1 \pm 7.5$ & $32.5 \pm 7.7$ & 0.28 \\
\hline$\%$ deficient $^{6}$ & 1.8 & 0.9 & 7.0 & 0.10 \\
\hline$\%$ marginal & 32.1 & 32.1 & 31.6 & \\
\hline
\end{tabular}




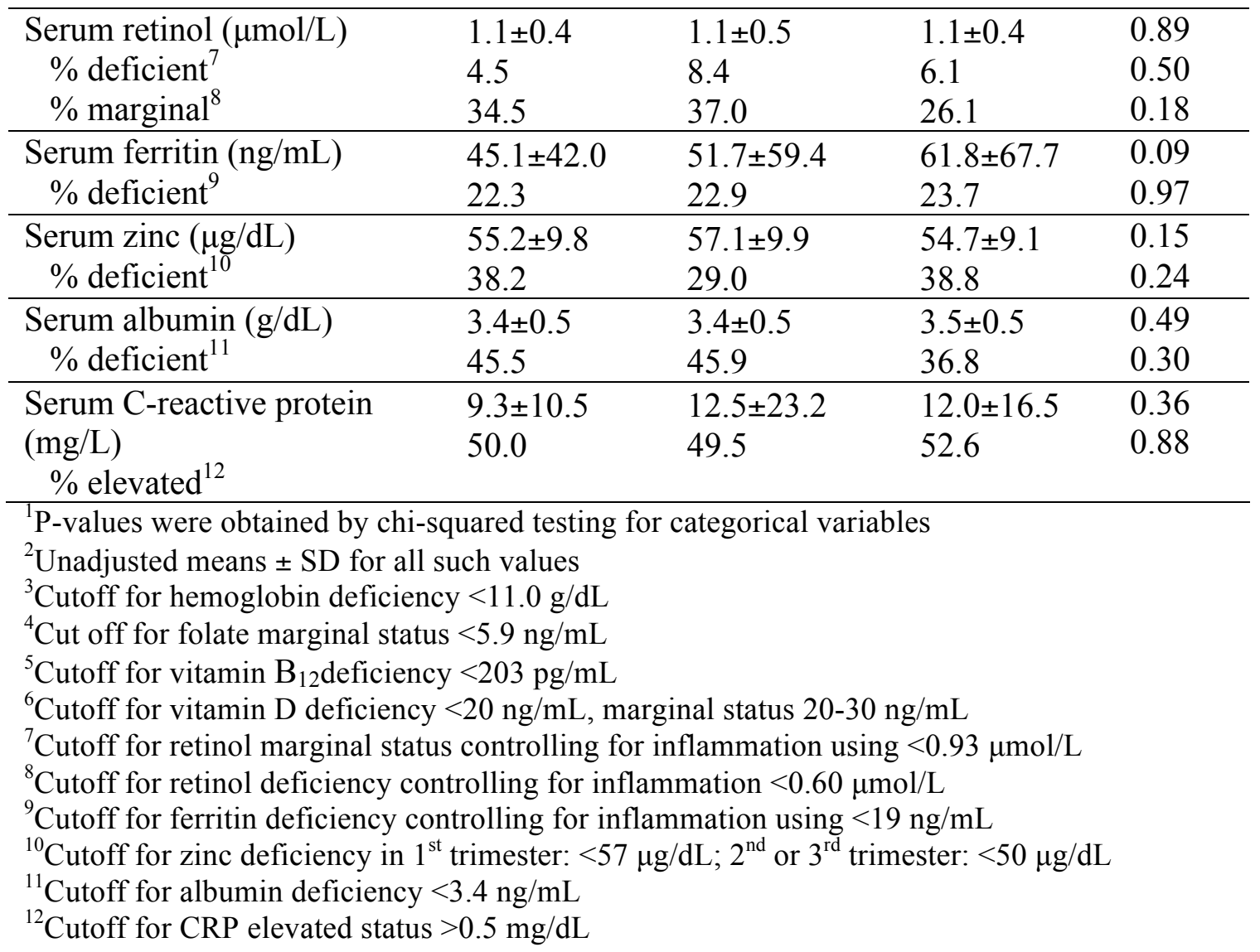

Adjusted baseline means of nutrients and proteins are described in Table 4.3. No significant differences were found between treatment groups.

Table 4.3 Baseline adjusted mean (SE) concentrations by treatment group ${ }^{1}$

\begin{tabular}{lllll}
\hline & $\begin{array}{l}\text { CSB-IFA } \\
(\mathrm{n}=112)\end{array}$ & $\begin{array}{l}\text { CSB- } \\
\text { UNIMMAP } \\
(\mathrm{n}=109)\end{array}$ & RUSF $(\mathrm{n}=114)$ & P-value $^{2}$ \\
& & $9.59(0.57)$ & $9.64(0.55)$ & 0.212 \\
\hline Folate $(\mathrm{ng} / \mathrm{mL})$ & $9.64(0.5)$ & $314.5(18.3)$ & $34.7(0.91)$ & 0.991 \\
${\text { Vitamin } \mathrm{B}_{12}(\mathrm{pg} / \mathrm{ml})^{3}}^{3}$ & $312.2(16.8)$ & 317 \\
Vitamin D $(\mathrm{ng} / \mathrm{mL})$ & $34.8(0.86)$ & $36.1(0.94)$ & $1.04(0.04)$ & 0.663 \\
Retinol $(\mu \mathrm{mol} / \mathrm{L})$ & $1.04(0.04)$ & $1.08(0.04)$ & $50.8(6.0)$ & 0.302 \\
Ferritin $(\mathrm{ng} / \mathrm{mL})$ & $41.9(5.7)$ & $41.6(6.2)$ & $53.1(1.1)$ & 0.449 \\
Zinc $(\mu \mathrm{g} / \mathrm{dL})$ & $53.2(1.0)$ & $54.6(1.2)$ & $3.5(0.06)$ & 0.586 \\
Albumin $(\mathrm{g} / \mathrm{dL})$ & $3.4(0.05)$ & $3.4(0.06)$ & $15.5(2.1)$ & 0.231 \\
CRP $(\mathrm{mg} / \mathrm{L})^{3}$ & $12.3(2.0)$ & $16.2(2.1)$ & & \\
\hline
\end{tabular}

${ }^{1}$ Covariates adjusted for include CRP concentration, fasting status, baseline MUAC, HIV status, parity, and gestational age

${ }^{2} \mathrm{P}$-value attained by ANOVA

${ }^{3} \mathrm{CRP}$ adjusted for baseline MUAC, HIV status, parity, and gestational age 
Analysis of nutrient concentration differences by treatment group was done in two ways, as described in Section 3.7. Table 4.4 shows adjusted mean concentrations at week 10, while controlling for covariates including baseline concentration, CRP at baseline and week 10, parity, HIV status and baseline MUAC.

Table 4.4 Adjusted least square mean concentrations (SE) at week 10 by treatment group $^{1}$

\begin{tabular}{|c|c|c|c|c|}
\hline & $\begin{array}{l}\text { CSB-IFA } \\
(\mathrm{n}=80)\end{array}$ & $\begin{array}{l}\text { CSB-UNIMMAP } \\
(\mathrm{n}=74)\end{array}$ & RUSF ( $\mathrm{n}=77)$ & P-value ${ }^{2}$ \\
\hline Folate $(\mathrm{ng} / \mathrm{mL})$ & $13.2(0.78)$ & $11.7(0.84)$ & $11.3(0.84)$ & 0.080 \\
\hline $\begin{array}{l}\text { Vitamin } \mathrm{B}_{12} \\
(\mathrm{pg} / \mathrm{ml})^{3,4}\end{array}$ & $255.4(11.3)$ & $281.5(12.3)$ & $310.4(12.3)$ & $<0.001$ \\
\hline Vitamin D $(\mathrm{ng} / \mathrm{mL})^{5}$ & $35.5(1.0)$ & $35.6(1.1)$ & $39.9(1.1)$ & $<0.001^{3}$ \\
\hline $\operatorname{Retinol}(\mu \mathrm{mol} / \mathrm{L})$ & $1.06(0.04)$ & $1.09(0.04)$ & $1.10(0.04)$ & 0.726 \\
\hline Ferritin $(\mathrm{ng} / \mathrm{mL})^{4}$ & $27.4(3.2)$ & $29.8(3.5)$ & $26.5(3.5)$ & 0.680 \\
\hline $\operatorname{Zinc}(\mu \mathrm{g} / \mathrm{dL})$ & 53.3( & 54.6( & 56.0 & 0.257 \\
\hline Albumin (g/dL) & $3.3(0.05)$ & $3.3(0.05)$ & $3.2(0$. & 0.326 \\
\hline $\mathrm{CRP}(\mathrm{mg} / \mathrm{L})^{6}$ & $9.7(3.4)$ & $13.1(3.7)$ & $11.3(3.7)$ & 0.698 \\
\hline Hemoglobin (g/dL) & $10.9(0.2)$ & $10.9(0.2)$ & $10.8(0.2)$ & 0.859 \\
\hline \multicolumn{5}{|c|}{$\begin{array}{l}{ }^{1} \text { Adjusted for week } 0 \text { concentration, week } 0 \text { CRP, week } 10 \text { CRP, fasting status, } \\
\text { gestational age, parity, baseline MUAC } \\
2 \text { P-value attained by ANOVA } \\
{ }^{3} \text { Interaction between time and treatment added into model } \\
{ }^{4} \text { Logarithmic transformation utilized for the p-values for vitamin } \mathrm{B}_{12} \text { and ferritin } \\
{ }^{5} \text { Vitamin D concentration also controlling for season of blood draw (rainy versus dry) } \\
{ }^{6} \mathrm{CRP} \text { controlling for CRP at week } 0 \text {, HIV status, parity, MUAC at enrollment }\end{array}$} \\
\hline \multicolumn{5}{|c|}{ Vitamins $\mathrm{B}_{12}$ and $\mathrm{D}$ concentrations at week 10 were significantly different } \\
\hline \multicolumn{5}{|c|}{ between treatment groups (Table 4.4). The subjects in the RUSF group had the greatest } \\
\hline \multicolumn{5}{|c|}{ vitamin $\mathrm{B}_{12}$ and $\mathrm{D}$ concentrations at week 10 , compared to CSB-UNIMMAP and CSB- } \\
\hline IFA. The other nutrie & nts and proteir & did not have sign & cant difference & etween \\
\hline
\end{tabular}

Adjusted mean change in concentration from week 0 to week 10 was analyzed using repeated measures MANOVA, controlling for the acute phase response via CRP at both time points (Table 4.5). Vitamin $\mathrm{B}_{12}$ was the only nutrient to maintain significance 
for both the effect of treatment and the effect of treatment over time. Mean change in vitamin D concentrations was not significantly different by treatment group; however, the effect of time and the interaction of time and treatment were significant for vitamin D $(\mathrm{p}<0.001)$. Ferritin also did not reach significance for differences in mean change by treatment, but the effect of time and the interaction of time and treatment were again significant $(\mathrm{p}=0.047)$. Almost all nutrients, except retinol, zinc, and CRP, had significant effects from the treatment over time.

Table 4.5 Mean change in adjusted concentrations from week 0 to week 10, by treatment group ${ }^{1}$

\begin{tabular}{lllllll}
\hline & $\begin{array}{l}\text { CSB-IFA } \\
(\mathrm{n}=80)\end{array}$ & $\begin{array}{l}\text { CSB- } \\
\text { UNIMMAP } \\
(\mathrm{n}=74)\end{array}$ & $\begin{array}{l}\text { RUSF } \\
(\mathrm{n}=77)\end{array}$ & $\begin{array}{l}\text { Treatment } \\
\text { effect } P\end{array}$ & $\begin{array}{l}\text { Time } \\
\text { effect } P\end{array}$ & $\begin{array}{l}\text { Time* } \\
\text { Treatment } \\
\text { effect } P\end{array}$ \\
\hline $\begin{array}{l}\text { Folate } \\
(\mathrm{ng} / \mathrm{mL})\end{array}$ & 4.0 & 1.7 & 2.2 & 0.365 & $<\mathbf{0 . 0 0 1}$ & 0.062 \\
$\begin{array}{l}\text { Vitamin } \mathrm{B}_{12} \\
(\mathrm{pg} / \mathrm{ml})^{2}\end{array}$ & -52.9 & -36.1 & -17.1 & $\mathbf{0 . 0 0 7}$ & $<\mathbf{0 . 0 0 1}$ & $\mathbf{0 . 0 0 2}$ \\
$\begin{array}{l}\text { Vitamin D } \\
(\mathrm{ng} / \mathrm{mL})\end{array}$ & 1.7 & 3.2 & 6.0 & 0.315 & $<\mathbf{0 . 0 0 1}$ & $<\mathbf{0 . 0 0 1}$ \\
$\begin{array}{l}\text { Retinol } \\
(\mu \mathrm{mol} / \mathrm{L})\end{array}$ & -0.06 & -0.02 & -0.01 & 0.955 & 0.182 & 0.604 \\
$\begin{array}{l}\text { Ferritin } \\
(\mathrm{ng} / \mathrm{mL})^{2}\end{array}$ & -18.0 & -17.8 & -23.1 & 0.812 & $<\mathbf{0 . 0 0 1}$ & $\mathbf{0 . 0 4 7}$ \\
$\begin{array}{l}Z \text { inc } \\
(\mu \mathrm{g} / \mathrm{dL})\end{array}$ & -2.7 & -2.1 & 1.4 & 0.394 & 0.472 & 0.072 \\
$\begin{array}{l}\text { Albumin } \\
(\mathrm{g} / \mathrm{dL})\end{array}$ & -0.19 & -0.12 & -0.25 & 0.961 & $<\mathbf{0 . 0 0 1}$ & 0.404 \\
$\mathrm{CRP}(\mathrm{mg} / \mathrm{L})$ & 1.8 & 1.2 & 1.9 & 0.157 & 0.372 & 0.984 \\
$\begin{array}{l}\text { Hemoglobin } \\
(\mathrm{g} / \mathrm{dL})\end{array}$ & 0.82 & 0.77 & 0.56 & 0.616 & $<\mathbf{0 . 0 0 1}$ & 0.588 \\
\hline
\end{tabular}

${ }^{\mathrm{l}}$ Least square means and p-values obtained from repeated measures MANOVA, controlling for CRP at both time points

${ }^{2}$ Logarithmic transformations used for vitamin $\mathrm{B}_{12}$ and ferritin

Change in percent deficient or of marginal status from week 0 to week 10 was analyzed, shown in Table 4.6. Inflammation cut-off values were used to determine 
deficiency and to control for the acute phase response with ferritin and retinol.

Inflammation was controlled for with zinc and albumin. Trimester of pregnancy at each

blood draw was also used to correct for differences in zinc deficiency.

Table 4.6 Percentage of deficiency or elevation by treatment at week 0 and week 10

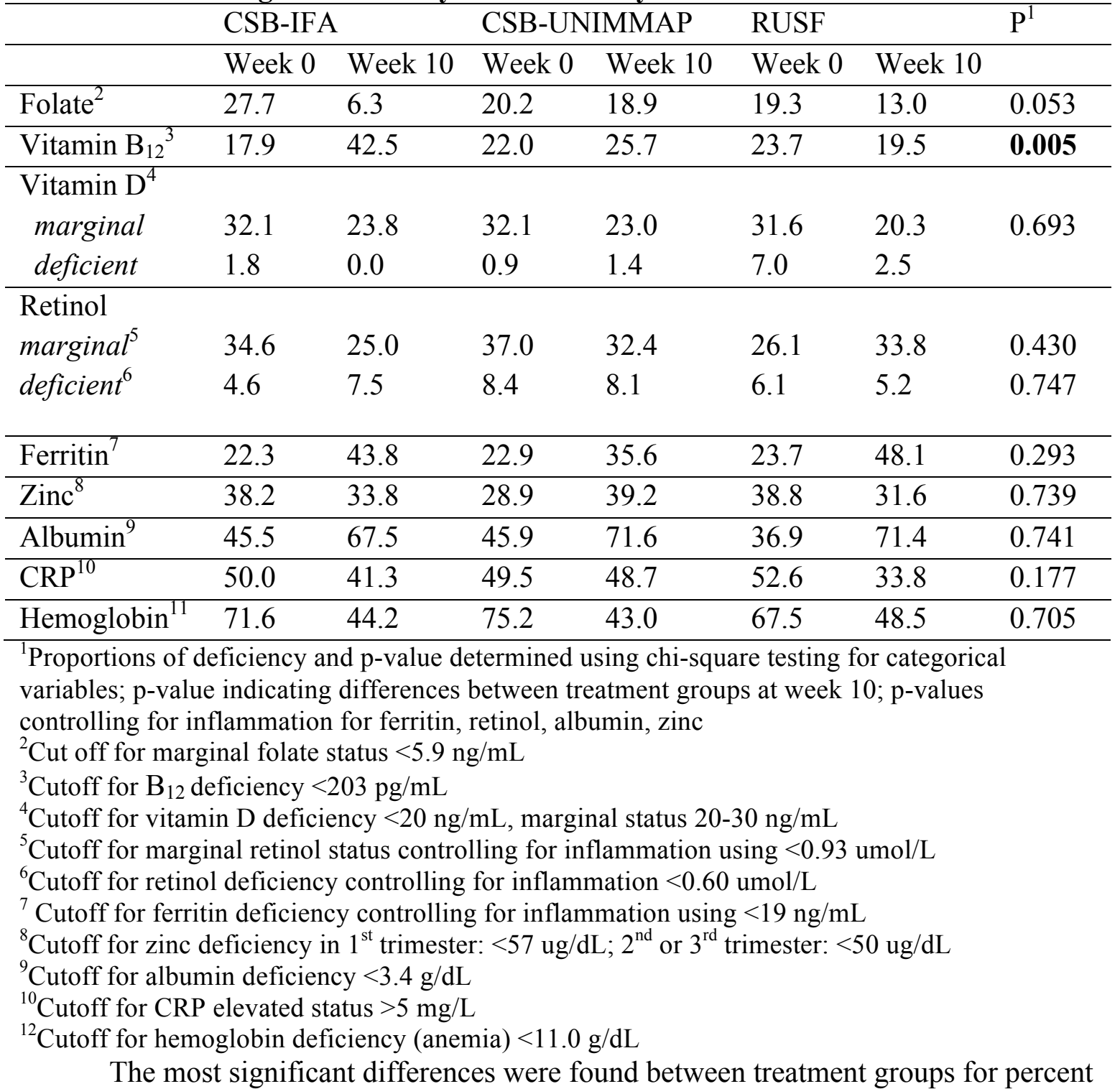

deficient in vitamin $B_{12}$ at week 10 (Table 4.6). All treatments saw a decrease in mean

vitamin $\mathrm{B}_{12}$ concentration and an increase in percentage deficient, however, the RUSF

group had the lowest proportion of women deficient in vitamin $\mathrm{B}_{12}$ at week $10(19.5 \%)$,

compared to $42.5 \%$ in the CSB-IFA group and $25.7 \%$ in the CSB-UNIMMAP group. At 
week 10, all groups had similar percentage with marginal vitamin D status, although the RUSF group experienced the greatest decrease in percentage points deficient from week 0 to week $10(-11.3 \%)$, compared to $-8.3 \%$ in the CSB-IFA group and $-9.1 \%$ in the CSBUNIMMAP group. The percentage of women deficient in vitamin D at week 10 was small and similar between groups. The proportion of women with marginal folate status at week 10 was the least in the CSB-IFA group (6.3\%), compared to $18.9 \%$ in the CSBUNIMMAP group and $13.0 \%$ in the RUSF group, which almost reached significance $(\mathrm{p}=0.056)$.

A two-way comparison using the Tukey analysis is shown in Table 4.7. Adjusted vitamin $B_{12}$ concentrations were significantly different between the RUSF and CSB-IFA groups $(\mathrm{p}<0.001)$ and between CSB-IFA and CSB-UNIMMAP $(\mathrm{p}=0.05)$. The RUSF group had the highest adjusted vitamin $\mathrm{B}_{12}$ concentrations at week 10. Adjusted vitamin D concentrations were significantly different between the RUSF and CSB-IFA groups $(\mathrm{p}<0.001)$ and between RUSF and CSB-UNIMMAP $(\mathrm{p}=0.019)$. No other nutrients or proteins were significantly different from each other in this analysis. 
Table 4.7 Adjusted means (least square) by treatment and two-way Tukey comparison of treatment effect between groups

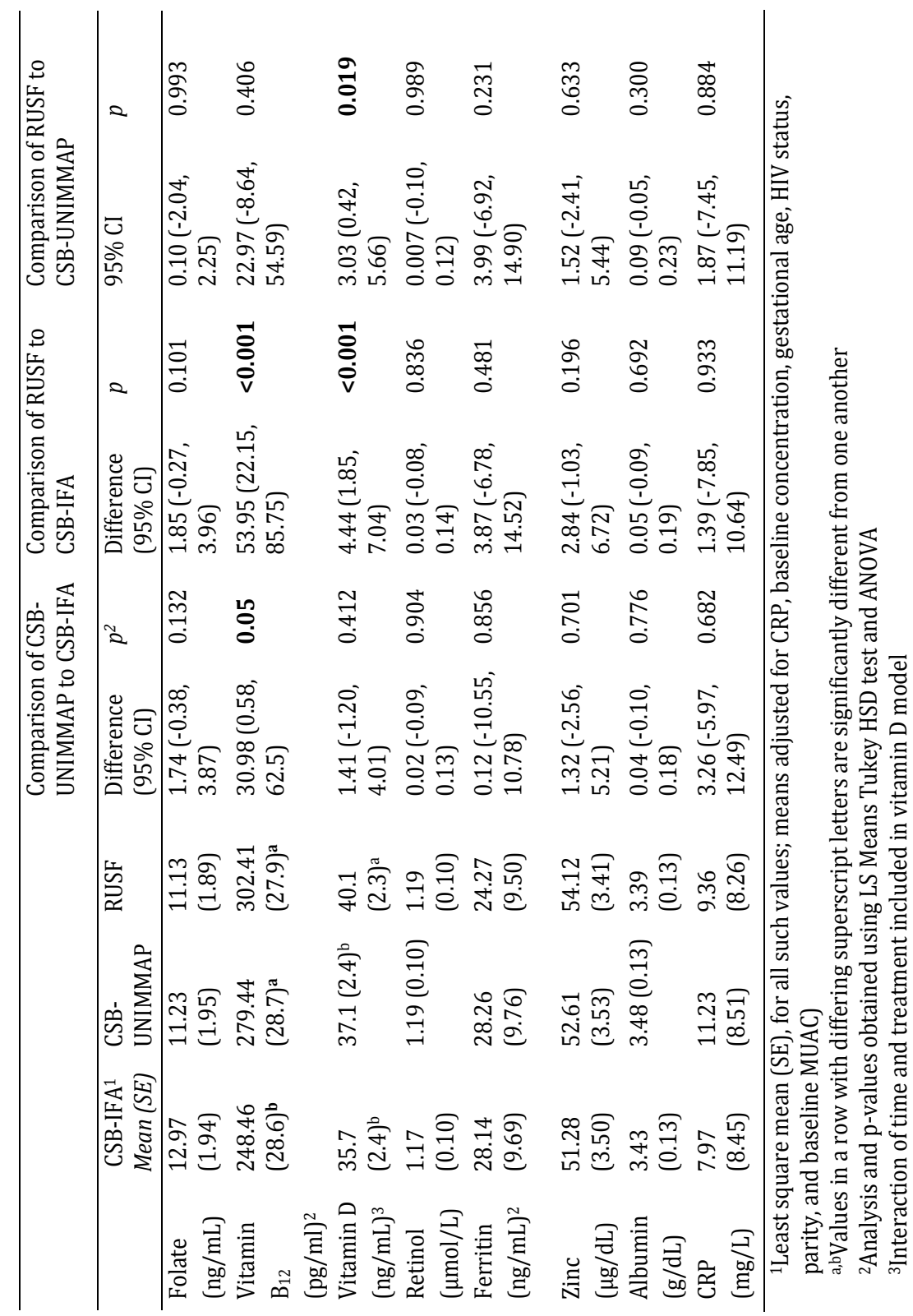




\subsection{Folate}

Folate concentrations had the greatest improvement in the CSB-IFA group (Table 4.5), followed by RUSF, then CSB-UNIMMAP (Figure 4.2). At baseline, CSB-IFA had the highest proportion of marginal folate status (27.7\%), compared to $20.2 \%$ in CSBUNIMMAP, and $19.3 \%$ in the RUSF group $(p=0.25)$. Differences between treatment groups in percentage with marginal folate status at week 10 approached significance, with RUSF decreasing to $6.3 \%$, and CSB-UNIMMAP and RUSF groups decreasing to $18.9 \%$ and $13.0 \%$, respectively $(\mathrm{p}=0.053$, Table 4.5$)$.

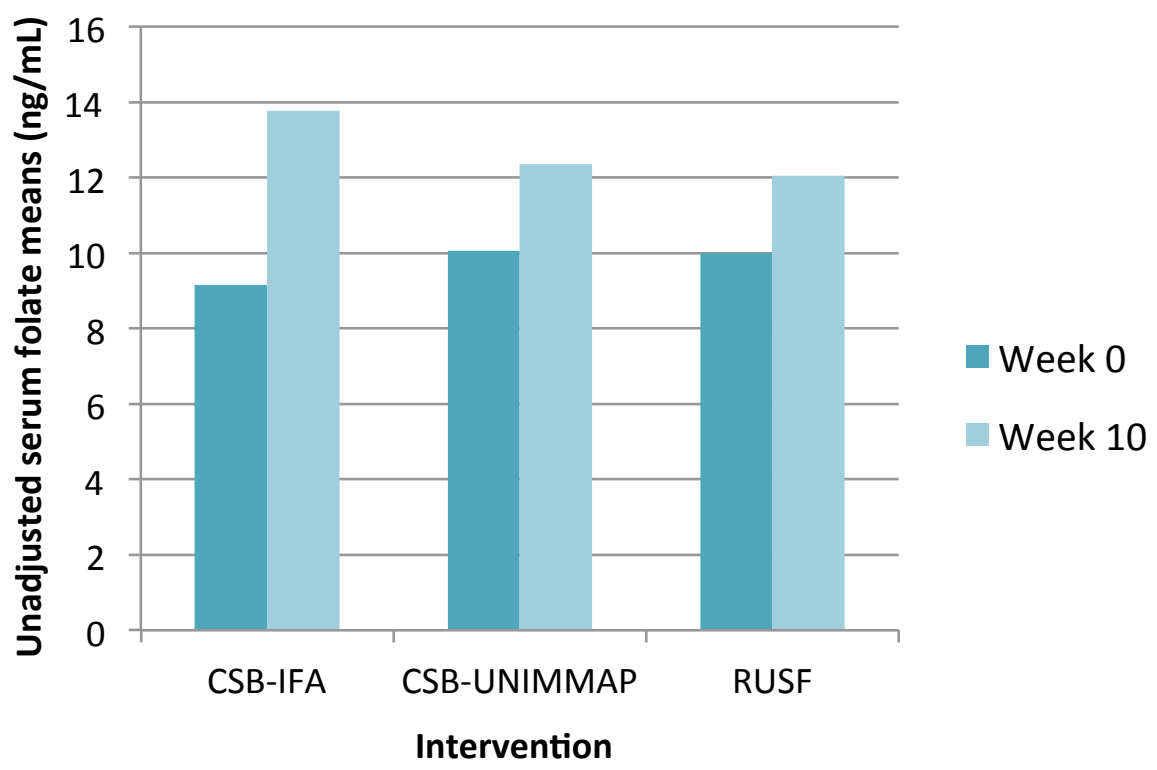

Figure 4.2 Change in folate means from week 0 to week 10 by treatment group.

Folate concentrations at baseline were similar by treatment group, but had significant differences based on gestational age at enrollment. Women who were further along in their pregnancy at enrollment had a lower mean folate concentration at baseline $(p=0.006)$. Folate concentrations at week 10 were not significantly affected by any other covariates, including CRP, parity, gestational age at enrollment, or HIV status. 


\subsection{Vitamin $B_{12}$}

Vitamin $\mathrm{B}_{12}$ decreased from week 0 to week 10 in all treatment groups, as is expected during pregnancy (van den Broek \& Letsky, 2000). The RUSF group experienced the smallest decrease throughout treatment $(-17.1 \mathrm{pg} / \mathrm{mL})$, compared to -36.1 $\mathrm{pg} / \mathrm{mL}$ in the CSB-UNIMMAP group and $-52.9 \mathrm{pg} / \mathrm{mL}$ in the CSB-IFA group $(\mathrm{p}=0.007)$ (Figure 4.3, Table 4.5). The effect of time and the effect of the interaction of time and treatment were also significant. CSB-IFA experienced the greatest increase in percentage of vitamin $\mathrm{B}_{12}$ deficiency (from $17.9 \%$ to $42.5 \%$ ), while CSB-UNIMMAP experienced a smaller increase $(22.0 \%$ to $25.7 \%)$. The RUSF group saw a significant decrease in percent deficient $(23.7 \%$ to $19.5 \%)$ (Table 4.6$)$.

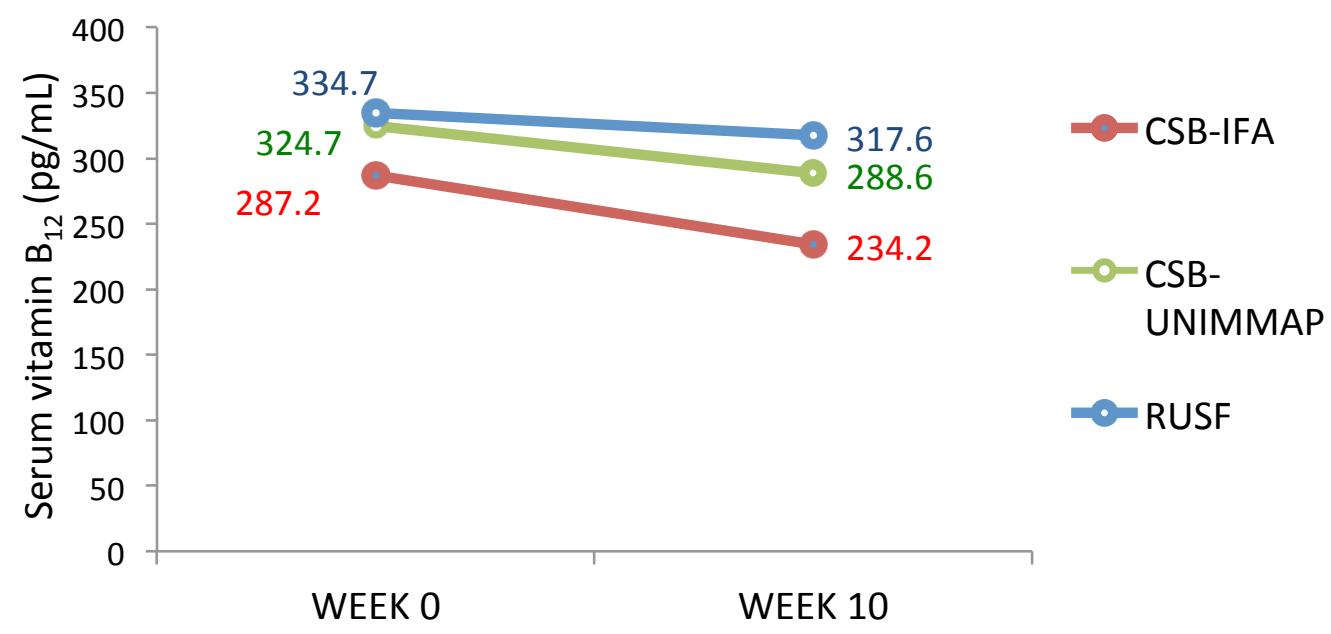

Figure 4.3 Change in adjusted mean vitamin $B_{12}$ concentrations $(\mathrm{pg} / \mathrm{mL})$ by treatment group

Vitamin $\mathrm{B}_{12}$ concentrations at week 0 and week 10 were highly correlated $\left(r^{2}=0.56, p<0.001\right)($ Figure 4.4$)$. The inflammatory response (CRP) was not significantly correlated to vitamin $\mathrm{B}_{12}$ concentrations at either time point $(\mathrm{p}=0.624$ at baseline and $\mathrm{p}=0.646$ at week 10). 


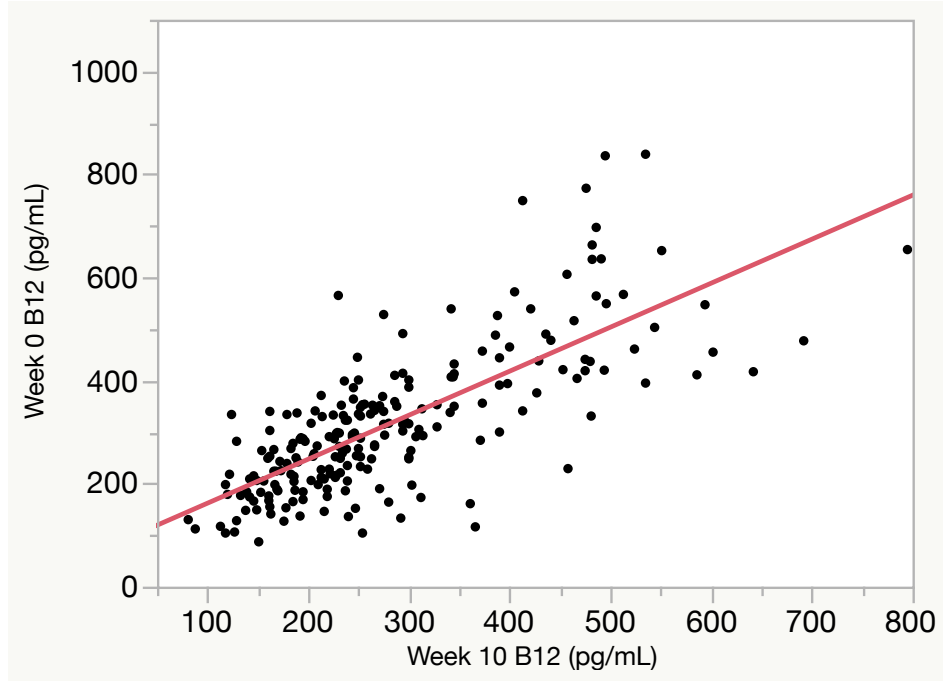

Figure 4.4 Correlation of week 0 to week 10 concentrations of vitamin $B_{12}(\mathrm{pg} / \mathrm{mL})$ $\left(\mathbf{p}<\mathbf{0 . 0 0 1}, \mathbf{r}^{2}=\mathbf{0 . 5 6}\right)$.

Two-way comparison indicated that vitamin $\mathrm{B}_{12}$ concentrations for RUSF were significantly different from CSB-IFA, and CSB-UNIMMAP concentrations were significantly different that CSB-IFA (Table 4.7). RUSF had a greater mean increase of $53.95 \mathrm{pg} / \mathrm{mL}$ compared to CSB-IFA (95\% CI 22.15, 85.75, $\mathrm{p}<0.001)$. CSB-UNIMMAP had a greater mean increase of $30.98 \mathrm{pg} / \mathrm{mL}$ than CSB-IFA (95\% CI 0.58, 62.5, $\mathrm{p}=0.05)$. Vitamin $\mathrm{B}_{12}$ concentrations were not significantly affected by any other covariates, including CRP, parity, gestational age at enrollment, baseline MUAC, and HIV status.

\subsection{Vitamin D}

Vitamin D concentrations were significantly greater in the RUSF group at week $10(39.9 \mathrm{ng} / \mathrm{mL})$, compared to the CSB-UNIMMAP (35.6 ng/mL) and CSB-IFA groups $(35.5 \mathrm{ng} / \mathrm{mL})(\mathrm{p}<0.001)$. Although rates of deficiency and marginal status were similar at week 10 (Table 4.6), the change in mean concentrations differed significantly (Figure 4.5). 


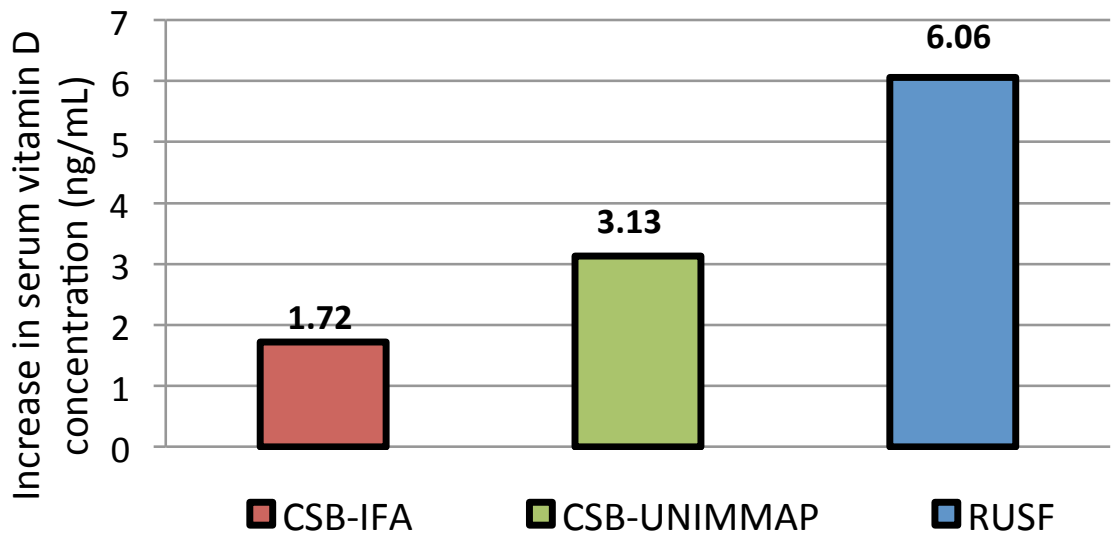

Figure 4.5 Mean increase in vitamin $D$ concentrations from week 0 to week 10 , by treatment group

There was a significant interaction found between time points and treatment for vitamin $\mathrm{D}$ (Table 4.5, $\mathrm{p}<0.001)$. This interaction was added into the ANOVA model analyzing differences between mean concentrations at week 10 (Table 4.4). Differences between the concentrations with and without the interaction added in the model are shown in Table 4.7. Both methods maintain a high level of significance.

Table 4.8 Difference in adjusted mean vitamin $D$ concentrations $(\mathrm{ng} / \mathrm{mL})$ with and without the interaction of time*treatment added into the model

\begin{tabular}{lllll}
\hline \multicolumn{5}{c}{ Least square mean (SE) } \\
\hline & CSB-IFA & CSB-UNIMMAP & RUSF & P \\
Vitamin D (ng/mL) & $35.1(2.4)$ & $36.5(2.4)$ & $39.3(0.1)$ & $<\mathbf{0 . 0 0 1}$ \\
$\begin{array}{l}\text { No interaction } \\
\text { Vitamin D (ng/mL) }\end{array}$ & $35.4(0.9)$ & $36.6(1.0)$ & $39.5(1.0)$ & $\mathbf{0 . 0 0 0 3}$ \\
Interaction & & & & \\
\hline
\end{tabular}

Vitamin D concentrations and clinic site were significantly related. When grouped into districts, significance was lost. Women from the clinic site Ngabu (Chikhwawa district) had significantly higher vitamin D concentrations at week 10 (when controlling for baseline concentration and all other covariates previously mentioned) than women from Matiya (Zomba district), Kambenje (Mulanje district), and Makwapala (Zomba 
district)(Table 4.8). The left column displays clinic site in decreasing order of serum vitamin D concentrations.

Table 4.9 Comparison of vitamin $D$ concentrations $(\mathrm{ng} / \mathrm{mL})$ at week 10 by clinic site and clinic district

\begin{tabular}{lll|lll}
\hline Clinic site $^{1}$ & $\begin{array}{l}\text { Vitamin } \\
\mathrm{D}^{2}\end{array}$ & $P$ & Clinic district & $\begin{array}{l}\text { Combined vitamin } \\
\mathrm{D} \mathrm{in} \mathrm{district}^{2}\end{array}$ & $P$ \\
\hline $\begin{array}{l}\text { Ngabu } \\
\text { (Chikhwawa) }\end{array}$ & 40.39 & 0.006 & Chikhwawa & 38.32 & 0.149 \\
$\begin{array}{l}\text { Matiya } \\
\text { (Zomba) }\end{array}$ & 33.43 & & Zomba & 36.64 & \\
$\begin{array}{l}\text { Kambenje } \\
\text { (Mulanje) }\end{array}$ & 32.71 & & Mulanje & 34.68 & \\
$\begin{array}{l}\text { Makwapala } \\
\text { (Zomba) }\end{array}$ & 30.95 & & Blantyre & 35.92 & \\
\hline
\end{tabular}

${ }^{1}$ Eleven other clinic sites excluded from data table (non-significant)

${ }^{2}$ Least square mean from ANOVA, controlling for baseline vitamin D, treatment group, parity, weeks gestation, and baseline MUAC

Maternal MUAC at baseline was significantly correlated with vitamin D

concentration at week 10, when controlling for baseline vitamin D, treatment group, HIV status, parity and gestational age $(\mathrm{p}=0.011)$. Women with a greater vitamin $\mathrm{D}$ concentration had lower baseline MUAC's (Figure 4.6). 


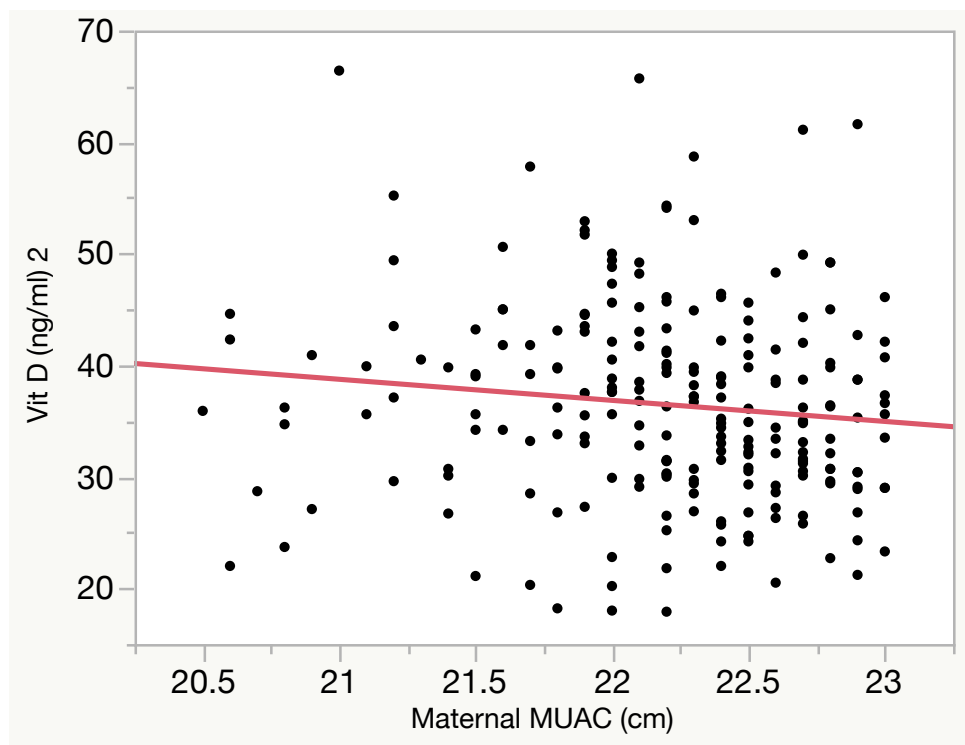

Figure 4.6 Correlation of baseline maternal MUAC and vitamin D concentration at week $10(p=0.011)$.

At baseline, vitamin D concentrations were significantly affected by gestational age and HIV status. Women who were 0.24 weeks further along in the pregnancy had a 1 $\mathrm{ng} / \mathrm{mL}$ increase in vitamin $\mathrm{D}$ concentrations $(\mathrm{p}=0.02)$. Interestingly, women who were HIV-positive had a higher baseline vitamin D concentration $(37.8 \mathrm{ng} / \mathrm{mL})$, when controlling for CRP and clinic site, compared to HIV-negative women (32.8 $\mathrm{ng} / \mathrm{mL})(\mathrm{p}<0.001)$. Vitamin D concentrations were significantly related to season (rainy season December-April and dry season May-November) for the second blood draw. Women who got their second blood drawn during the rainy season had significantly greater vitamin D concentrations, when controlling for other covariates $(39.7 \mathrm{ng} / \mathrm{mL})$, compared to $34.4 \mathrm{ng} / \mathrm{mL}$ in women who got their second blood drawn in the dry season $(p<0.001)$. At baseline, seasonality did not affect vitamin D concentrations. Vitamin D 
concentrations at week 10 were not significantly related to any other covariates, including CRP, parity, gestational age at enrollment, or HIV status.

\subsection{Retinol}

Retinol did not differ significantly by treatment group with any form of analysis. Mean adjusted concentrations at week 10 were almost identical, at 1.06, 1.09, and 1.10 $\mu \mathrm{mol} / \mathrm{L}$ for CSB-IFA, CSB-UNIMMAP, and RUSF, respectively ( $\mathrm{p}=0.726)$. All treatment groups saw a small and similar decrease in mean concentration from week 0 to week $10(-0.06,-0.02$, and $-0.01 \mu \mathrm{mol} / \mathrm{L}$ for CSB-IFA, CSB-UNIMMAP, and RUSF, respectively $[p=0.955])$. Changes in percent deficient and percent with marginal status differed non-significantly by treatment group (Figure 4.7).

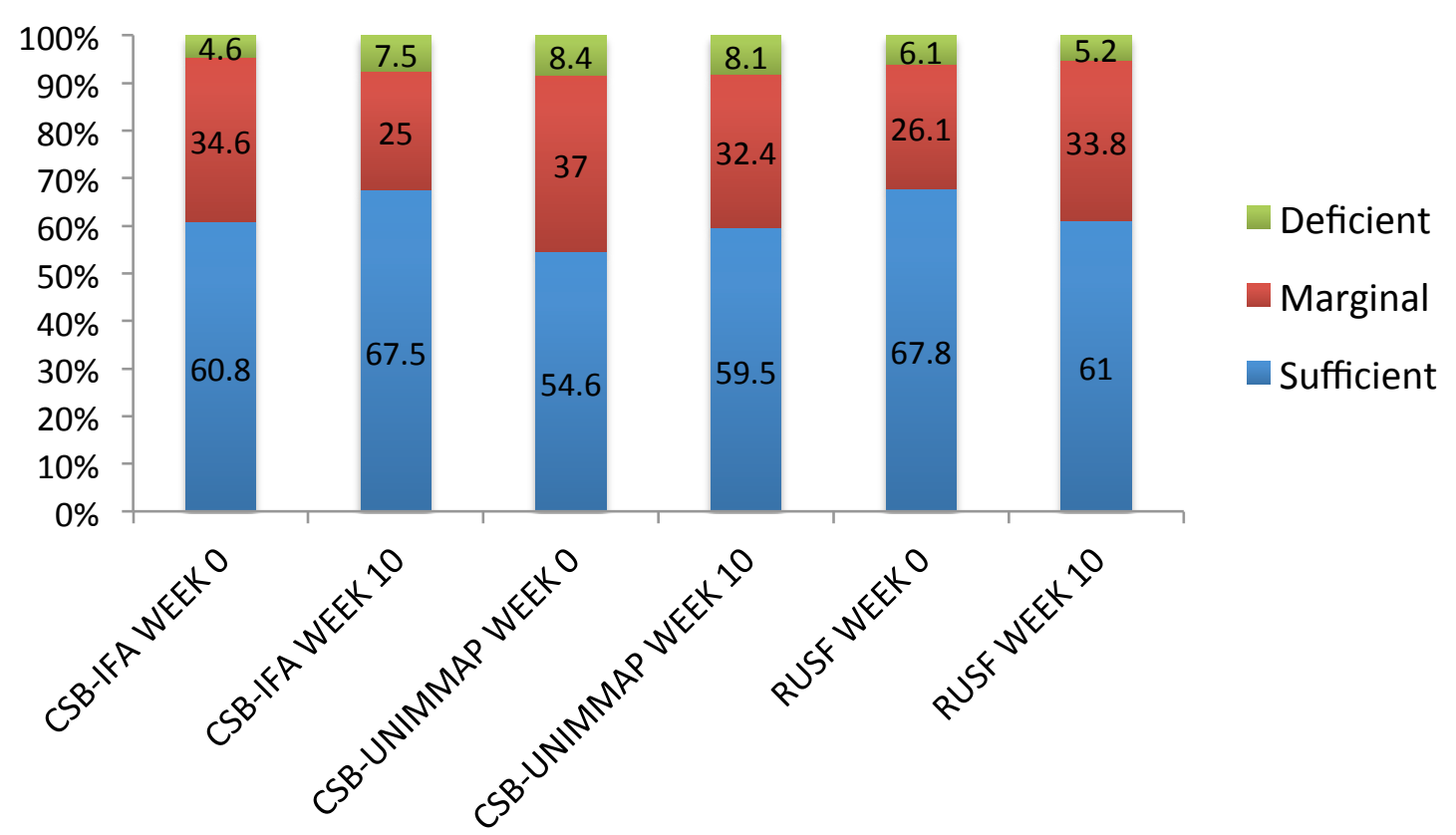

Figure 4.7 Percent of deficient, marginal, and sufficient retinol status by treatment group from week 0 to week 10.

Baseline retinol concentrations were related to HIV status. HIV-positive women had a significantly lower baseline retinol mean $(0.96 \mu \mathrm{mol} / \mathrm{L})$, compared to HIV-negative 
women $(1.13 \mu \mathrm{mol} / \mathrm{L})$, when controlling for CRP concentration $(\mathrm{p}=0.007)$. Retinol at week 10 was not significantly related to HIV status. From week 0 to week 10 , HIVpositive women saw an improvement in mean retinol from $0.96 \mathrm{umol} / \mathrm{L}$ to $1.05 \mathrm{umol} / \mathrm{L}$, while HIV-negative women had constant retinol concentrations $(1.13 \mathrm{umol} / \mathrm{L}$ at both time points).

The RUSF group experienced an increase in percentage with marginal retinol status (26.1 to $33.8 \%)$, but a decrease in percentage with deficient status (6.1 to $5.2 \%$, non-significant)(Table 4.9). RUSF was the only group to show a decrease in percentage deficient from week 0 to week 10 . However, RUSF was also the only group to experience a decrease in percentage with sufficient retinol status. None of these shifts in proportions were significant.

Table 4.10 Proportions of sufficient, marginal, and deficient retinol status by treatment group, from week 0 to week 10 , controlling for inflammation.

\begin{tabular}{lllllll}
\hline & CSB-IFA & \multicolumn{3}{l}{ CSB-UNIMMAP } & RUSF & \\
\hline Retinol & Week 0 & Week 10 & Week 0 & Week 10 & Week 0 & Week 10 \\
\hline Sufficient (\%) & 60.8 & 67.5 & 54.6 & 59.5 & 67.8 & 61.0 \\
Marginal (\%) & 34.6 & 25.0 & 37.0 & 32.4 & 26.1 & 33.8 \\
Deficient (\%) $^{3}$ & 4.6 & 7.5 & 8.4 & 8.1 & 6.1 & 5.2 \\
\hline
\end{tabular}

${ }^{1}$ Cutoff for sufficient retinol status $>0.94 \mu \mathrm{mol} / \mathrm{L}$

${ }^{2}$ Cutoff for marginal retinol status controlling for inflammation using $<0.93 \mu \mathrm{mol} / \mathrm{L}$

${ }^{3}$ Cutoff for retinol deficiency controlling for inflammation $<0.60 \mu \mathrm{mol} / \mathrm{L}$

Retinol concentrations at baseline were significantly and negatively correlated with CRP concentrations (Figure 4.8) $(\mathrm{r}=0.13 ; \mathrm{p}<0.0001)$. Retinol concentrations are expected to decrease during an inflammatory response, which is indicated in this population as well. At week 10, retinol concentrations and CRP concentrations were also correlated significantly, although not as strongly as with the baseline concentrations $(\mathrm{r}=0.08, \mathrm{p}<0.0001)$ 


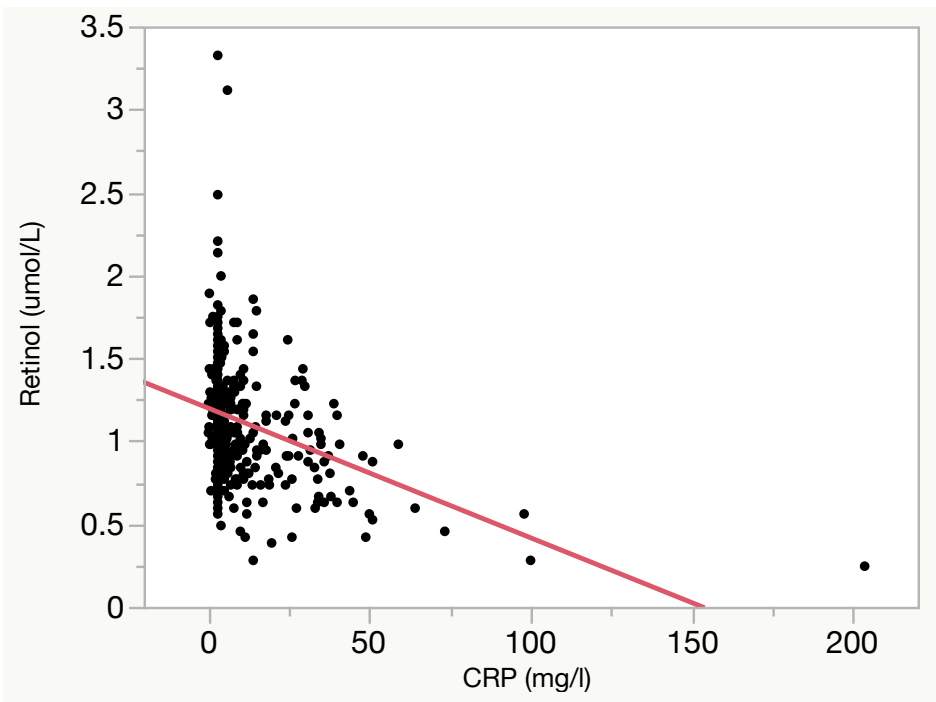

Figure 4.8 Correlation of baseline retinol $(\mu \mathrm{mol} / \mathrm{L})$ and baseline CRP $(\mathrm{mg} / \mathrm{L})$ $(\mathbf{p}<0.0001)$.

\subsection{Ferritin}

Ferritin concentrations at baseline did not differ significantly by treatment group. Adjusted ferritin concentrations at week 10 were similar between groups: 27.4, 29.8, and $26.5 \mathrm{ng} / \mathrm{mL}$ for CSB-IFA, CSB-UNIMMAP, and RUSF, respectively $(\mathrm{p}=0.245)($ Table 4.4). Ferritin concentrations decreased throughout treatment for all treatment groups (Table 4.5). The RUSF group had the greatest decrease from week 0 to week 10 (-23.1 $\mathrm{ng} / \mathrm{mL}$ ), compared to $-17.8 \mathrm{ng} / \mathrm{mL}$ in the CSB-UNIMMAP group and $-18.0 \mathrm{ng} / \mathrm{mL}$ in the CSB-IFA group $(\mathrm{p}=0.812)$. Significant interactions between time and treatment were observed for the analysis of change in ferritin concentrations (Table 4.5).

Percentages of women deficient in ferritin at baseline were similar among treatment groups (22.3-23.7\%). At week 10, the percentage had increased for all groups, with RUSF having the greatest amount of deficiency (48.1\%), compared to $35.6 \%$ in the CSB-UNIMMAP group and $43.8 \%$ in the CSB-IFA group. These changes in percent deficient were not significant between treatment groups (Table 4.6). 
Baseline ferritin concentrations were significantly related to CRP concentrations, parity, and HIV status. Women with a higher CRP had significantly higher ferritin concentrations $(\mathrm{p}<0.0001)$. Women with higher parity had significantly lower mean ferritin concentrations $(\mathrm{p}=0.005)$ (Figure 4.9). In analysis using parity as a categorical variable, the effect remained significant. Primiparous women had significantly higher baseline ferritin concentrations (mean $64.4 \mathrm{ng} / \mathrm{mL}$ ), compared to multiparous women (mean $39.0 \mathrm{ng} / \mathrm{mL})(\mathrm{p}<0.0001)$. HIV-positive women had a significantly lower baseline mean ferritin $(34.98 \mathrm{ng} / \mathrm{mL})$, compared to HIV-negative women $(54.76 \mathrm{ng} / \mathrm{mL})(\mathrm{p}=0.031)$.

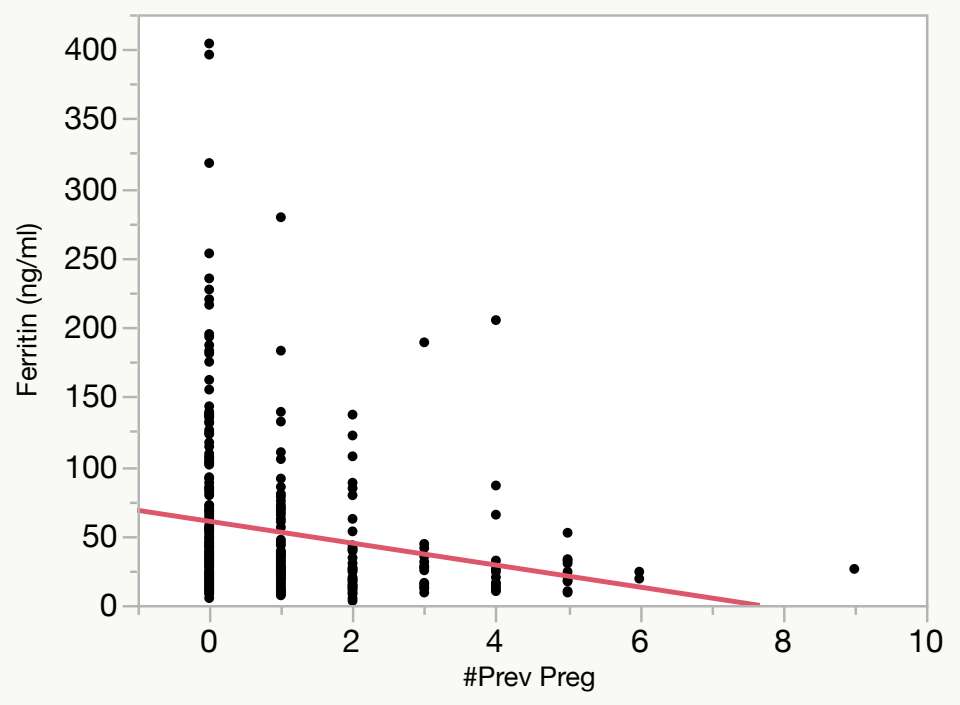

Figure 4.9 Correlation of parity and baseline ferritin concentrations $(\mathrm{ng} / \mathrm{mL})(\mathrm{p}<0.0001)$

Ferritin was significantly and positively correlated to CRP, at both baseline and week 10. Figure 4.10 depicts the correlation between ferritin and CRP at week 10 $\left(\mathrm{r}^{2}=0.44, \mathrm{p}<0.0001\right)$. Ferritin concentrations are expected to increase with the inflammatory response. 


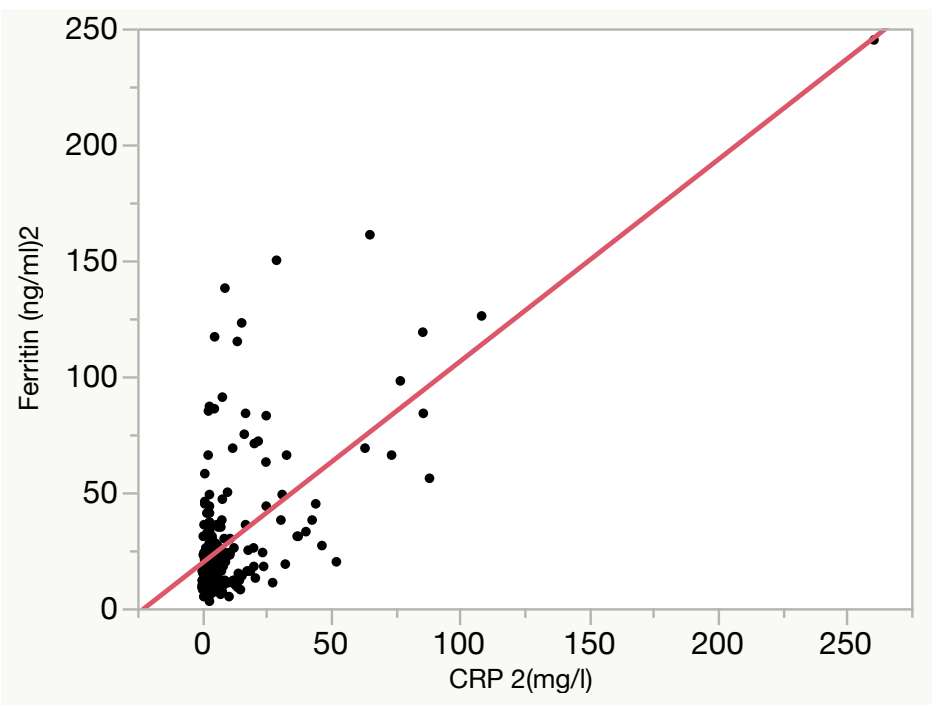

Figure 4.10 Correlation of week 10 ferritin $(\mathrm{ng} / \mathrm{mL})$ and week 10 CRP $(\mathrm{mg} / \mathrm{L})(\mathbf{p}<\mathbf{0 . 0 0 0 1})$.

\subsection{Zinc}

Zinc concentrations did not differ significantly by treatment group. All treatment groups experienced high rates of deficiency at baseline $(38.2 \%, 29.0 \%$, and $38.8 \%$ deficient in the CSB-IFA, CSB-UNIMMAP, and RUSF groups, respectively)(Table 4.2). Zinc concentrations decreased from week 0 to week 10 in the CSB-IFA $(-2.7 \mu \mathrm{g} / \mathrm{dL})$ and CSB-UNIMMAP $(-2.1 \mu \mathrm{g} / \mathrm{dL})$ groups, but not in the RUSF group, which experienced an increase of $1.4 \mu \mathrm{g} / \mathrm{dL}$ (Table 4.5, Figure 4.11). The effect of treatment was not significant $(p=0.394)$, although the interaction between time and treatment approached significance $(p=0.072)$

Baseline zinc concentrations were significantly correlated to gestational age at enrollment. Women who had a higher gestational age had significantly lower zinc concentrations at baseline $(\mathrm{p}=0.0002)$. Fasting status impacted zinc concentrations at baseline. Women who were in a fasted state had significantly higher baseline zinc concentrations $(56.9 \mu \mathrm{g} / \mathrm{dL})$, compared to $50.7 \mu \mathrm{g} / \mathrm{dL}$ in women who were not fasted at 
the time of blood draw $(\mathrm{p}<0.001)$. Zinc concentrations at baseline and week 10 were not significantly affected by other covariates, including CRP, HIV status, parity, or baseline MUAC. Rates of zinc deficiency increased $10.3 \%$ in the CSB-UNIMMAP group, while the RUSF and CSB-IFA groups saw a decrease in deficiency rates of $7.2 \%$ and $4.4 \%$, respectively $(\mathrm{p}=0.604)$.

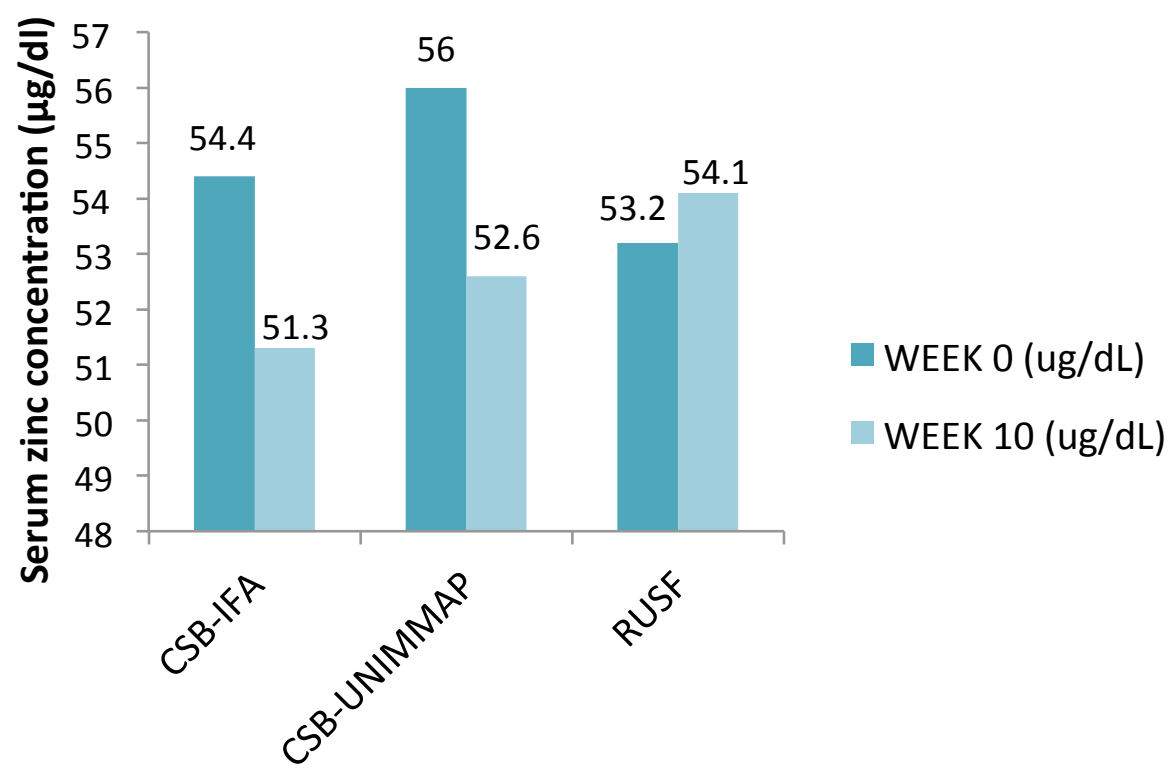

Figure 4.11 Adjusted serum zinc concentrations at baseline and week 10 by treatment group

Zinc concentrations were significantly related to hemoglobin concentrations, when controlling for CRP and gestational age. Higher hemoglobin concentrations at baseline and week 10 were correlated with higher zinc concentrations at baseline and week 10 ( $\mathrm{p}=0.015$ and $\mathrm{p}=0.009$, respectively). A $1.23-\mathrm{g} / \mathrm{dL}$ increase in hemoglobin concentration correlated to $1 \mathrm{ug} / \mathrm{dL}$ increase in zinc concentration at week 10. Ferritin and zinc were also related. Those with greater ferritin concentrations were more likely to have greater zinc concentrations at baseline $(\mathrm{p}=0.0014)$; this association did not remain significant at week 10 . 


\subsection{Albumin}

Albumin concentrations did not differ significantly by treatment group. Concentrations decreased from week 0 to week 10 by $0.19,0.12$, and $0.25 \mathrm{~g} / \mathrm{dL}$ in the CSB-IFA, CSB-UNIMMAP, and RUSF groups, respectively. The effect of treatment was not significant ( $p=0.961)$, although the effect of time was significant $(p<0.001$, Table 4.5). At week 10, all groups had high rates of hypoalbuminemia: $67.5 \%, 71.6 \%$, and $71.4 \%$ in the CSB-IFA, CSB-UNIMMAP, and RUSF groups, respectively $(p=0.818)$. Baseline means were close to the cutoff value for hypoalbuminemia of $3.4 \mathrm{~g} / \mathrm{dL}$, thus the small decrease that each group experienced correlated to a large increase in percent deficient.

Albumin at baseline was significantly and negatively correlated to CRP concentration $(\mathrm{p}=0.001)$. Women with lower albumin concentrations at baseline were more likely to have higher CRP concentrations. Albumin is a negative acute phase responder and is expected to decrease during an inflammatory response. Albumin at week 10 was not related to CRP at week $10(p=0.243)$.

\subsection{C-reactive protein}

Mean C-reactive protein (CRP) concentrations were elevated at week 0 and week 10. All treatment groups saw a small and non-significant increase in CRP concentrations. However, the percentage of women with elevated CRP status decreased from week 0 to week 10 by $8.7 \%, 0.8 \%$, and $18.8 \%$ in the CSB-IFA, CSB-UNIMMAP, and RUSF groups, respectively ( $\mathrm{p}=0.177)$. As discussed in previous sections, CRP was significantly correlated with retinol, ferritin, and albumin concentrations. CRP at week 10 was significantly correlated with CRP at baseline. 
CRP was related to HIV status, when controlling for parity and recent illness. At baseline, HIV-infected women had a mean CRP of $17.6 \mathrm{mg} / \mathrm{L}$, compared to $10.7 \mathrm{mg} / \mathrm{L}$ in HIV-uninfected women $(\mathrm{p}=0.025)$. However, the amount of HIV-positive women in this population was small $(\mathrm{n}=38)$. CRP was also related to self-reported infection with malaria within the last two months. Women who answered "yes" to having malaria within the previous two months had a significantly greater mean CRP (19.6 mg/L) compared to women who did not have malaria $(8.65 \mathrm{mg} / \mathrm{L})(\mathrm{p}<0.0001)$. Reported pneumonia and diarrhea were not related to CRP.

CRP was not affected by clinic site or district, parity, education level, age, or weeks of gestation at enrollment.

\subsection{Hemoglobin}

Mean adjusted hemoglobin concentrations at baseline were 9.8, 9.8, and 10.2 g/dL for the CSB-IFA, CSB-UNIMMAP, and RUSF groups, respectively ( $p=0.135)$. Overall, $71 \%$ of women were anemic, based on hemoglobin concentrations $<11 \mathrm{~g} / \mathrm{dL}$.

Hemoglobin at baseline was significantly related to baseline ferritin levels. Interestingly, baseline hemoglobin and ferritin were negatively correlated. Women with higher hemoglobin were more likely to have a lower ferritin at baseline $(\mathrm{p}<0.001)($ Figure 4.12). Women with a lower gestational age were more likely to have a greater baseline hemoglobin concentration $(\mathrm{p}=0.03)$. Baseline hemoglobin was slightly higher in women with greater MUAC, although this did not reach significance $(\mathrm{p}=0.055)$. Parity was related to baseline hemoglobin; women who were primiparous had lower hemoglobin concentrations (mean $9.7 \mathrm{~g} / \mathrm{dL})$ compared to multiparous women $(10.1 \mathrm{~g} / \mathrm{dL})(\mathrm{p}=0.03)$. Baseline hemoglobin was significantly related to clinic district. Women in the Zomba 
district had the highest baseline hemoglobin concentrations (10.5 g/dL), compared to Mulanje (10.0 g/dL), Chikhwawa (9.6 g/dL), and Blantyre (9.6 g/dL)(p<0.001). This effect did not remain significant at week 10 hemoglobin concentrations $(\mathrm{p}=0.051)$.

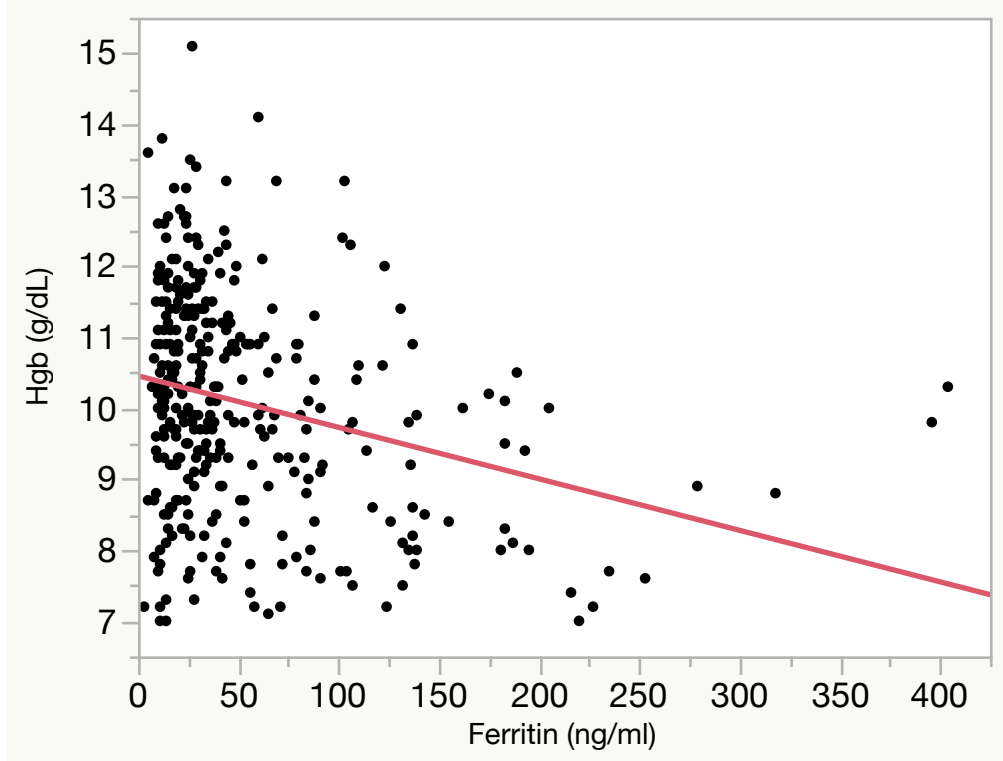

Figure 4.12 Correlation of baseline hemoglobin $(\mathrm{g} / \mathrm{dL})$ to baseline ferritin $(\mathrm{ng} / \mathrm{mL})$

Hemoglobin at week 10 increased for all groups to $10.9,10.8$, and $10.8 \mathrm{~g} / \mathrm{dL}$ for CSB-IFA, CSB-UNIMMAP, and RUSF groups, respectively ( $\mathrm{p}=0.819)$. Week 10 hemoglobin was negatively and non-significantly related to ferritin at week 10, although it approached significance $(\mathrm{p}=0.051)$. Women who were HIV-positive had nonsignificantly lower mean hemoglobin concentrations at week $10(10.5 \mathrm{~g} / \mathrm{dL})$ compared to HIV-negative women $(11.1 \mathrm{~g} / \mathrm{L})(\mathrm{p}=0.09)$.

Hemoglobin was related to albumin concentrations at baseline. Women with a greater hemoglobin concentrations of $1 \mathrm{~g} / \mathrm{dL}$ were related to an increase in albumin concentrations of $0.59 \mathrm{~g} / \mathrm{dL}$ at baseline ( $\mathrm{p}=0.0004)$, when controlling for gestational age, 
parity, ferritin and CRP concentrations. The trend still remained at week 10, but not was significant $(\mathrm{p}=0.08)$.

\subsection{Birth outcomes}

Birth measurements of weight, length, MUAC, and head circumference were not significantly different between treatment groups (Table 4.10). Three-month measurements of weight, length, and head circumference were not significantly different. Measurements from twin births were removed $(n=1)$. Overall prevalence of low birth weight $(<2500 \mathrm{~g})$ was $35.1 \%$, with no significant differences between treatment groups.

Table 4.11 Unadjusted mean \pm SD (n) of birth and 3-month measurements by treatment group

$\begin{array}{llll}\text { CSB-IFA } & \text { CSB-UNIMMAP } & \text { RUSF } & P^{1}\end{array}$

\begin{tabular}{lllll}
\hline Birth weight $^{2}$ & $2.67 \pm 0.38(94)$ & $2.65 \pm 0.36(91)$ & $2.63 \pm 0.49(91)$ & 0.825 \\
3 month weight & $5.42 \pm 0.81(93)$ & $5.67 \pm 0.74(92)$ & $5.52 \pm 0.65(89)$ & 0.067 \\
Birth length & $46.97 \pm 2.18(94)$ & $46.76 \pm 1.93(88)$ & $46.51 \pm 2.42(90)$ & 0.356 \\
3 month length & $58.3 \pm 2.89(91)$ & $58.3 \pm 2.29(93)$ & $58.1 \pm 2.49(88)$ & 0.767 \\
Birth MUAC & $9.33 \pm 0.09(94)$ & $9.38 \pm 0.09(90)$ & $9.33 \pm 0.09(90)$ & 0.927 \\
3-month MUAC & $12.9 \pm 1.12(93)$ & $13.3 \pm 1.0(93)$ & $13.0 \pm 0.92(88)$ & $0.017 *$ \\
Birth HC & $34.14 \pm 1.54(94)$ & $34.05 \pm 1.36(91)$ & $33.94 \pm 1.75(91)$ & 0.678 \\
3 month HC & $40.2 \pm 1.8(92)$ & $40.4 \pm 1.4(92)$ & $40.5 \pm 1.7(89)$ & 0.529 \\
3 month Hgb & $9.5 \pm 1.4(93)$ & $9.5 \pm 1.7(89)$ & $9.6 \pm 1.3(85)$ & 0.879
\end{tabular}

${ }^{1} \mathrm{P}$-value obtained by Student's t-test

${ }^{2}$ Weight $(\mathrm{kg})$

${ }^{3}$ Length $(\mathrm{cm})$

${ }^{4}$ MUAC: mid-upper arm circumference $(\mathrm{cm})$

${ }^{5} \mathrm{HC}$ : head circumference $(\mathrm{cm})$

${ }^{6} \mathrm{Hgb}$ : Hemoglobin (g/dL) 
Infant MUAC at 3 months was significantly related to treatment group, with infants of mothers from the CSB-UNIMMAP having the greatest 3-month MUAC. However, the significance was lost when birth MUAC and maternal MUAC were controlled for $(\mathrm{p}=0.087)$.

Maternal MUAC was not significantly related to birth weight, length, MUAC, or head circumference. There was a significant relationship between 3-month infant weight and baseline maternal weight $(\mathrm{p}<0.001)$, when accounting for infant birth weight.

Some correlations were found between maternal nutrient concentrations and infant measurements. Baseline maternal ferritin concentrations (when controlling for CRP) were significantly and negatively related to infant birth weight $(\mathrm{p}=0.036)$. When parity was added into the model, significance lost $(\mathrm{p}=0.17)$. However, 3 -month infant weight was positively and significantly related to baseline maternal ferritin $(p=0.017)$ and had similar loss of significance when parity was added into the analysis $(\mathrm{p}=0.13)$.

Folate concentration at week 10 approached a significant relationship with birth length, when controlling for parity and CRP at week $10(\mathrm{p}=0.07)$. The trend did not remain for 3-month infant length.

Interestingly, birth length was significantly and negatively related to retinol concentration at week 10, when controlling for CRP at both time points and parity. When retinol concentrations at week 10 were decreased, mean infant birth length was increased $(\mathrm{p}=0.045)$. This trend did not remain significant for 3-month infant length. No other nutrients were significantly related to birth weight or birth length at either time point. Infant 3-month hemoglobin was significantly and positively associated with maternal baseline hemoglobin concentration $(\mathrm{p}=0.0497)$. Significance was lost when 
maternal week 10 hemoglobin was added as a covariate. Ferritin at either time point was not significantly related to infant 3-month hemoglobin.

Infant birth weight, length, and head circumference were not significantly associated with CRP concentration at baseline or week 10. Analysis was done using CRP both as a continuous variable and as a categorical variable (elevated versus non-elevated). Infant birth MUAC approached significance when CRP was used as a categorical variable. Women with an elevated CRP at week 10 were more likely to have an infant with a lower birth MUAC ( $\mathrm{p}=0.056)$. Forty-seven percent of women who had elevated CRP at week 10 had infants with LBW ( $\mathrm{p}=0.11)$.

Interestingly, HIV-infected women were significantly more likely to have an infant with greater birth weight (mean $2.86 \mathrm{~kg}$ ) compared to HIV-uninfected women (mean $2.68 \mathrm{~kg}$ ), when adjusting for CRP, treatment group, and parity ( $\mathrm{p}=0.049)$. This did not remain significant with 3-month infant weights.

Infant birth MUAC was slightly negatively correlated to baseline maternal vitamin $\mathrm{D}$, however, the significance was lost when controlling for $\mathrm{CRP}(\mathrm{p}=0.09)$. There was a trend of a lower baseline vitamin D concentration correlating with a higher infant birth MUAC. Infant MUAC was not significantly related to any other nutrient measures at baseline or week 10. Infant head circumference at birth was not significantly related to any baseline or week 10 nutrient concentrations in the mother. 


\section{CHAPTER 5: DISCUSSION}

In this randomized, researcher-blinded controlled clinical trial, we found that treatment with the macro- and micronutrient fortified RUSF for 10 weeks improved serum vitamins $\mathrm{B}_{12}$ and $\mathrm{D}$ status in moderately malnourished pregnant women more than treatment with a fortified corn-soy blended flour plus a prenatal multiple micronutrient supplement (CSB-UNIMMAP) or a fortified corn-soy blended flour with iron and folic acid supplementation (CSB-IFA). Serum folate concentrations had the greatest improvement after treatment with CSB-IFA. Changes in serum ferritin, retinol, zinc and albumin concentrations were not significantly different between treatment groups. Despite provision with $200 \%$ the RDA (or greater) for most micronutrients, inflammation and deficiency rates for all nutrients measured except vitamin $\mathrm{B}_{12}$ and folate remained high and were changed little by any intervention.

This population experienced overall high rates of deficiency at baseline in zinc (35.4\%), ferritin (22.9\%), vitamin $\mathrm{B}_{12}(21.2 \%)$, hemoglobin $(71.3 \%)$, and albumin (42.7\%). Marginal proportions of vitamin D (31.9\%), retinol (32.4\%) and folate $(22.4 \%)$ were also prevalent. Primiparous women had significantly higher baseline ferritin concentrations. An acute phase response was present in more than half of the women (50.7\%), as measured by a CRP concentration $>5 \mathrm{mg} / \mathrm{L}$. At week 10 , the most significant

differences between groups were found for serum vitamins $\mathrm{B}_{12}$ and $\mathrm{D}$, with women in the RUSF group experiencing the greatest improvement in these nutrients.

\subsection{Folate}

The CSB-IFA group experienced the greatest increase in folate concentrations, followed by RUSF, then CSB-UNIMMAP. Differences between the treatment groups at 
week 10 and change in percent with marginal status from week 0 to week 10 approached significance, with CSB-IFA experiencing the greatest improvements in both. None of the women were deficient in folate at baseline therefore marginal status was assessed instead $(<5.9 \mathrm{ng} / \mathrm{mL})$. While approximately $70 \%$ of women reported taking supplements at baseline, only $2 \%$ reported taking folic acid supplements (the rest iron).

The overall mean serum concentration at baseline was $9.7 \mathrm{ng} / \mathrm{mL}$. In contrast, a study in anemic southern Malawian pregnant women found that the mean serum folate concentration was $7.8 \mathrm{ng} / \mathrm{mL}$, which is markedly lower than the folate concentrations in this study (van den Broek \& Letsky, 2000). Using cut-off values of both $4.1 \mathrm{ng} / \mathrm{mL}$ and $3.4 \mathrm{ng} / \mathrm{mL}$ for deficiency, van den Broek \& Letsky found that $34 \%$ and $21 \%$ of the women had a folate deficiency, respectively. The more stringent criteria were used to account for hemodilution during pregnancy. There are several reasons for this better folate status in the present study. The women in the van den Broek study may have had a larger proportion with folate deficiency because they were all anemic, which increases the likelihood of deficiency in more than one nutrient (van den Broek \& Letsky, 2000). However, $71 \%$ of women in the present study were anemic at baseline. Second, folate status may be worsened by malarial infection (van den Broek \& Letsky, 2000). At baseline in the present study, $15.2 \%$ of women reported having malaria within the two previous months, and at the time of enrollment, $8 \%$ of women were taking malarial treatment. In the van den Broek study, $8 \%$ of women had malaria parasites in their blood (van den Broek \& Letsky, 2000).

Women who had a greater gestational age at baseline had lower folate concentrations, which is consistent with hemodilution throughout pregnancy. Greater 
maternal folate concentrations at week 10 were related to a nearly significant trend of greater infant birth length $(\mathrm{p}=0.052)$.

Although the proportion of those with marginal folate status decreased in all treatment groups, the largest decrease was in the CSB-IFA group. It is possible that the CSB-IFA group experienced the greatest benefit from supplementation because the amount of folic acid present in the CSB-IFA and CSB-UNIMMAP treatments (659 ug) were higher than the amount in RUSF (574 ug) (Appendix B).

Supplementation with folic acid during pregnancy is universally recommended, in order to reduce the risk of neural tube defects and congenital abnormalities (FAO \& WHO, 1998). However, coverage of supplements at antenatal care clinics (ANC) may not be reaching all pregnant women in the areas. In this study, only $2 \%$ of women reported taking folic acid supplements at baseline, indicating that the clinics may not have adequate supplies to consistently give out to pregnant women.

In this population, neither the RUSF nor CSB-UNIMMAP treatments improved folate status more than the CSB-IFA group. However, folate concentrations were relatively high to begin with and no subject had deficient status during this study.

\subsection{Vitamin $B_{12}$}

Vitamin $\mathrm{B}_{12}$ deficiency was found in $21.2 \%$ of women at baseline. This is similar to the previously mentioned study in Malawi (van den Broek \& Letsky, 2000), which found that $16-33 \%$ of anemic pregnant women had a deficiency in vitamin $\mathrm{B}_{12}$ (depending on the cut-off value that was used). A systematic review and meta-analysis by Sukumar, et al (2016) found that the average vitamin $\mathrm{B}_{12}$ insufficiency from 57 studies worldwide was at $21 \%, 19 \%$, and $29 \%$ in the first, second and third trimesters, 
respectively, which is in a consistent range with the population in the present study (Sukumar et al., 2016). The RUSF group was the only treatment to have a significant decrease in percentage deficient from week 0 to week 10, whereas CSB-UNIMMAP and CSB-IFA both increased their percentage of women deficient throughout the study.

Vitamin $\mathrm{B}_{12}$ concentrations were significantly different between treatment groups at week 10, with women in the RUSF group having the greatest improvements. Vitamin $\mathrm{B}_{12}$ concentrations are known to decrease throughout pregnancy due to a trans-placental transfer of the vitamin close to term (van den Broek \& Letsky, 2000). There was a decrease in vitamin $\mathrm{B}_{12}$ from week 0 to week 10 in all treatment groups; however, the RUSF group experienced the smallest decrease and CSB-IFA experienced the greatest decrease.

The fact that at week $10,42.5 \%$ of the women in the CSB-IFA group were classified as vitamin $\mathrm{B}_{12}$ deficient is important (compared to $19.5 \%$ in the RUSF group). A Nepalese study of pregnant women found that those who were vitamin $\mathrm{B}_{12}$ deficient had a two-fold increased likelihood of preeclampsia and preterm delivery (Bondevik et al., 2001). Maternal vitamin $B_{12}$ deficiency is also linked to poor fetal growth and brain development (Neumann et al., 2014). The meta-analysis by Sukumar, et al (2016) found that the odds ratio of women with insufficient vitamin $\mathrm{B}_{12}$ status having a low birth weight (LBW) or small for gestational age (SGA) infant was 1.70 (95\% CI: 1.16, 2.50) (Sukumar et al., 2016). Although this sub-study did not assess adverse pregnancy and birth outcomes, birth measurements were available for majority of the subjects' infants and were not significantly related to maternal vitamin $B_{12}$ concentration. It is possible that the inconsistency of results from this study compared to others is due to the small sample 
size $(n=276)$ producing inadequate power to detect a difference in birth measurements. A similarly powered study randomized 366 women in India to receive vitamin $\mathrm{B}_{12}$, iron and folate, or just iron and folate during pregnancy. Vitamin $\mathrm{B}_{12}$ concentrations were significantly greater in both mother and infant in the vitamin $B_{12}$ group, however, infant birth weight was not significantly different between groups (Duggan et al., 2014).

The association of insufficient vitamin $\mathrm{B}_{12}$ status and pregnancy complications, including preeclampsia and preterm birth, are typically mediated by the elevated homocysteine concentration that accumulates when vitamin $\mathrm{B}_{12}$ is low (Sukumar et al., 2016). The present study did not measure serum homocysteine concentrations, so this factor is unknown.

The prevalence of low vitamin $\mathrm{B}_{12}$ concentrations at baseline may be related to low dietary intake of vitamin $\mathrm{B}_{12}$, which is only found in animal products. It is likely that these women have infrequent access to animal proteins, contributing to low vitamin $\mathrm{B}_{12}$ status. Commonly consumed foods per day in Malawi include nsima (maize porridge), beans, rice, and some fruits and vegetables. A study done in HIV-positive pregnant women in Malawi analyzed dietary patterns, wealth and anthropometrics, and found that women with greater wealth were more likely to consume an animal-based diet, compared to lower wealth women consuming mostly plant-based or grain-based diets (Ramlal et al., 2015). While that study did not analyze serum or dietary vitamin $B_{12}$ concentrations, it is likely that women consuming the plant- or grain-based diets typical of Malawian women in the present study were consuming low levels of dietary vitamin $B_{12}$. Another study in Malawian women found that the median intake of protein from animal sources was 5.6 
grams per day (out of a median of 46.9 grams total protein per day)(Gibson \& Huddle, 1998).

Although maternal vitamin $\mathrm{B}_{12}$ concentrations did not produce significant effects on infant birth measurements, it is possible that vitamin $\mathrm{B}_{12}$ supplementation may have long-term effects on the child. One study found that children of mothers who had the lowest vitamin $\mathrm{B}_{12}$ concentrations at 28 weeks gestation had lower attention and memory test score performance compared to children of mothers with the highest vitamin $\mathrm{B}_{12}$ concentrations (Bhate, Deshpande, Bhat, Joshi, \& Ladkat, 2009). Another study found that higher vitamin $\mathrm{B}_{12}$ and lower homocysteine concentrations in children were related to higher mental development scores at age 12-18 months (Strand et al., 2013). Vitamin $\mathrm{B}_{12}$ is a crucial nutrient for pregnancy, fetal, birth, and child outcomes in later life. Results from this study and others indicate that the addition of vitamin $\mathrm{B}_{12}$ to standard antenatal supplementation practices may be warranted.

\subsection{Vitamin D}

The RUSF group experienced significant increases in vitamin D concentrations, compared to CSB-UNIMMAP and CSB-IFA. Only three percent of women in this population were severely vitamin D deficient, however, marginal status affected approximately one-third of women at baseline. The RUSF group saw the greatest increase in serum vitamin D concentration, compared with a lesser increase in the CSBUNIMMAP and CSB-IFA groups. Vitamin D content of the treatment foods was slightly different, with RUSF containing $30 \mu \mathrm{g}$, CSB-UNIMMAP containing $30.9 \mu \mathrm{g}$, and CSBIFA containing $25.4 \mu \mathrm{g}$ vitamin D. 
Although all treatment groups contained approximately double the RDA for vitamin D during pregnancy, the RUSF group contained $2.26 \mathrm{~g}$ of alpha-linolenic acid (omega-3 fatty acid) and $13.96 \mathrm{~g}$ of linoleic acid (omega-6 fatty acid). Vitamin D is a fatsoluble nutrient; therefore absorption of vitamin D from the treatment food may have been better facilitated by the RUSF. Intake of fat from other dietary sources is not likely to be high, due to majority of Malawian diets being higher in grains and other carbohydrates. The study by Ramlal et al. (2015) found that average fat intake in Malawian women consuming a grain-based diet was $19.9 \mathrm{~g} /$ day (Ramlal et al., 2015).

There is some discrepancy in the literature regarding cut-off values to use for vitamin D deficiency and insufficiency, which can lead to challenges in assessing similarities and differences between studies. The present study utilized the more lenient cut-off value of $<20 \mathrm{ng} / \mathrm{mL}$ classified as deficient and $20-30 \mathrm{ng} / \mathrm{mL}$ classified as marginal status.

Serum vitamin D concentration in this study was significantly related to clinic location. The participants were sampled from 15 antenatal clinics, which were grouped into 4 districts. The clinic site that had significantly higher week 10 vitamin D concentrations was Ngabu (Chikhwawa district), which is a rural area in a valley which experiences higher temperatures and less shade than the other districts. Women from Ngabu experienced almost a $10 \mathrm{ng} / \mathrm{mL}$ increase in vitamin D concentrations at week 10 compared to women from the Makwapala clinic in Zomba district, which is in the highlands. The other clinic districts of Mulanje and Blantyre are in the mountains and urban settings, respectively. The differences in vitamin D concentrations from women of differing geographic areas in Malawi are interesting, and could be due to time spent in the 
sun and amount of clothing worn. Women in the Chikhwawa district generally are walking or working during the day for long periods of time and wear less protective clothing compared to women in the more urban areas of Blantyre. Dietary vitamin D was likely not different between the clinic sites, as Malawian food does not typically contain much vitamin D. Important sources of vitamin D are fatty fish, eggs, animal products, and fortified foods.

Two relationships were unexpected and interesting. First, there was a negative correlation between maternal baseline MUAC and week 10 vitamin D concentrations, meaning that women with a lower MUAC at baseline had higher vitamin D levels at week 10, regardless of treatment group. There was no correlation between baseline MUAC and baseline vitamin D concentrations. This could indicate that women who were less nourished at baseline were able to benefit more from supplementation than betternourished women, thus producing a greater increase in vitamin D concentrations in those women. Second, HIV-positive women had significantly higher baseline vitamin D concentrations compared to HIV-negative women, which was not affected by CRP concentration. It is possible that women with HIV have differences in diet or exposure to sun compared to women without HIV. However, the reasons for these findings are unknown and warrant further research.

Inadequate maternal vitamin $\mathrm{D}$ during pregnancy has been linked to adverse birth outcomes, including LBW (Thorne-Lyman \& Fawzi, 2013), premature (Bodnar et al., 2013) and SGA infants (Aghajafari et al., 2013). Low maternal vitamin D stores are related to impaired infant growth measurements in other studies. A trial by Kalra et al. (2012) found that women receiving vitamin D during pregnancy had infants with 
significantly higher birth weights, lengths, and head circumference compared to women not receiving vitamin D treatment (Kalra et al., 2012). Again, the present study did not have adequate power to determine if birth measurements had significant differences between treatment groups.

The differences in vitamin D concentrations at week 10 between treatment groups are important when considering the effect that adequate maternal vitamin D stores can have on the health of both mother and infant. While the relationship between maternal MUAC and vitamin D concentration deserves further research, these results suggest that treatment with the RUSF containing omega- 3 and omega- 6 fatty acids may improve vitamin D status better than treatments with similar vitamin D content but containing little fat.

\subsection{Retinol}

Retinol status was not significantly changed by any treatment. Approximately $11 \%$ of the study population was deficient in retinol at baseline $(<0.70 \mu \mathrm{mol} / \mathrm{L})$, while approximately $50 \%$ of the population had marginal retinol status $(<1.05 \mu \mathrm{mol} / \mathrm{L})$. These results are similar to an analysis done by WHO with data from 1995-2005, which estimated that $13.7 \%$ of pregnant Malawian women had a deficiency in vitamin A $(<0.70$ $\mu \mathrm{mol} / \mathrm{L})(\mathrm{WHO}, 2009)$. At week 10 in the present study, retinol concentrations were very similar between treatment groups and the change in percent deficient or marginal was small. The vitamin A content in the treatment foods was similar, although the CSBUNIMMAP treatment contained the highest amount (3210 ug), compared to $2628 \mathrm{ug}$ in the RUSF treatment and 2410 ug in the CSB-IFA treatment. 
A study assessed the effect of prenatal supplementation with LNS, MMN or IFA on breast milk retinol (Klevor et al., 2016). Results were similar to the present study, in that there were no significant differences in retinol between treatment groups. Although the assessment method was different than the present study (breast milk versus serum retinol), the results were consistent in that a lipid-based supplement did not have any additional benefit above a MMN supplement. These authors conclude that dietary vitamin A intake may have been sufficient in this population of Ghanaian women (Klevor et al., 2016). Common vitamin A or beta-carotene containing foods include leafy green vegetables, red or orange vegetables, sweet potatoes, and red palm oil. Mangoes are also a good source of vitamin A, which are commonly consumed in Malawi.

A study by Kumwenda et al. (2002) in 697 Malawian HIV-infected pregnant women found contrasting results to the present study. These women were randomized to receive either 10,000 IU (3000 ug) vitamin A plus iron and folic acid daily during pregnancy, or just iron and folic acid. Women in the vitamin A group had significantly higher plasma vitamin A concentrations at 38 weeks gestation and higher breast milk retinol compared to women receiving only iron and folic acid (Kumwenda et al., 2002). It's possible that the present study did not see a significant improvement in retinol status because the mean retinol concentration at baseline was already sufficient $(1.16,1.19$ and 1.19 for CSB-IFA, CSB-UNIMMAP, and RUSF, respectively). The percentage of retinol-deficient women in the CSB-IFA group did increase by $2.9 \%$ from week 0 to week 10, while the percentage of retinol-deficient women in the RUSF group decreased by $0.9 \%$ (non-significantly). This could indicate that the most deficient women were able to see a benefit from the lipid-containing RUSF. 
Another interesting result was that retinol at week 10 was negatively correlated to infant birth length $(\mathrm{p}=0.027)$. When controlling for CRP concentration, HIV status and parity, significance was reduced but still maintained $(\mathrm{p}=0.04)$. It is unclear why a higher maternal retinol would correlate to a reduced infant birth length. In contrast, the study in Malawi by Kumwenda et al. (2002) found that infants of mothers who were supplemented with vitamin A during pregnancy had a significantly greater length at 6 weeks compared to infants of mothers receiving iron and folic acid $(\mathrm{p}=0.03)$, although this significance was lost when infants were measured at 14 weeks and 6 months (Kumwenda et al., 2002).

Retinol concentrations at baseline and week 10 were highly negatively correlated with CRP concentration, as is expected with the inflammatory response (Thurnham \& Mccabe, 2012). HIV status was also related to baseline retinol concentration. Women who were HIV-positive were more likely to have lower retinol status, compared to HIVnegative women, even when controlling for CRP concentration. The relationship was not significant for week 10 concentrations because HIV-positive women saw a greater improvement in retinol concentrations than HIV-negative women. Although nonsignificant, the improvement of mean retinol concentrations in HIV-positive women from $0.96 \mu \mathrm{mol} / \mathrm{L}$ to $1.05 \mu \mathrm{mol} / \mathrm{L}$ is clinically relevant, as that changes the overall classification of HIV-positive women in this study from marginal status to sufficient status. It is possible that HIV-positive women experienced the greatest benefit from vitamin A supplementation due to their compromised immune systems. Vitamin A is highly involved in immune function. People with vitamin A deficiency are more likely to develop infections, and infections can in turn increase the risk of vitamin A deficiency 
(Christian et al., 1998). HIV-infected women with vitamin A deficiency are more likely to have a LBW infant and have increased risk of mother-to-child transmission (MTCT) of HIV (Semba et al., 1995). In the present study, MTCT was not measured and birth weight was not affected by retinol status. However, there was a relationship between birth weight and HIV status in that HIV-positive women were more likely to have infants with greater birth weight compared to HIV-negative women, which is inconsistent with the literature. However, when maternal retinol concentration was added as a covariate into the model, significance was lost, indicating that retinol concentration may play a role in infant birth weight.

Supplementation with a lipid-containing RUSF did not improve retinol concentrations in pregnant women more than supplementation with a multiple micronutrient tablet or iron and folic acid, all containing similar amounts of vitamin A. Infants of mothers with higher retinol concentrations were more likely to have a reduced birth length, although more trials with a larger sample size would be required to confirm these results.

\subsection{Ferritin}

Serum ferritin concentrations decreased from week 0 to week 10 in all treatment groups, although none of the differences were significant between groups. Approximately one-quarter of this population was ferritin deficient at baseline, which increased to $43.8 \%, 35.6 \%$, and $48.1 \%$ in the CSB-IFA, CSB-UNIMMAP, and RUSF groups, respectively. The amount of iron in the CSB-IFA treatment $(79.2 \mathrm{mg}$ ) was almost double the content of the CSB-UNIMMAP and RUSF treatments (45.3 $\mathrm{mg}$ and $45 \mathrm{mg}$, 
respectively). Despite the high amount of iron present in all treatment groups, ferritin concentrations were not improved.

The decrease in ferritin concentrations in this population has also been noted in the literature. Serum ferritin tends to decrease between weeks 12 and 25 of gestation due to expansion of maternal red blood cell mass, thus making assessment of deficiency difficult (Allen, 2000; Kaestel, Aaby, Ritz, \& Friis, 2015). Most of the iron transfer to the fetus occurs after week 30 of pregnancy, further reducing maternal iron stores (Allen, 2000).

In this population, women with a higher CRP had significantly higher ferritin concentrations, as is expected with the inflammatory response (Kaestel et al., 2015). A cross-sectional study of pregnant women in Guinea-Bissau found that one-third of the subjects had low ferritin concentrations, which were significantly associated with stage of pregnancy. Women above 29 weeks gestation had up to $59 \%$ lower ferritin concentrations compared to women under 16 weeks gestation (Kaestel et al., 2015). In the present study, gestational age was not related to ferritin concentration.

Parity was significantly associated with ferritin. Primiparous women had significantly higher ferritin concentrations at baseline compared to women with higher parity. More pregnancies are likely to result in the mother becoming nutritionally depleted, especially if the pregnancies are closely spaced together (Kaestel et al., 2015). A contrasting study in Zimbabwean pregnant women found that higher parity (gravida >3) women had 1.21 times higher ferritin concentrations compared to primiparous women. This study also found that ferritin concentrations were lower in women with 
gestational age of 32-35 weeks, compared to women of 22-25 weeks gestation (Friis et al., 2001).

Since CRP is a marker for an inflammatory response, it is logical that a woman with an infection would have higher CRP concentrations, thus higher ferritin concentrations. In the population studied by Friis et al., women with malarial parasitemia had a 2.25 times higher serum ferritin concentration compared to women without malaria (Friis et al., 2001). The relationship between high iron stores in the body and increased susceptibility to malaria has been studied. A study in Malawian women found that those with malaria had better iron stores than those without malarial infection (Senga et al., 2011). In the present study, ferritin and hemoglobin concentrations at baseline and week 10 were not significantly related to malarial infection within the previous two months, when controlling for CRP concentrations, HIV status and parity.

HIV-infected women in this population had significantly lower ferritin concentrations at baseline compared to HIV-uninfected women. Friis et al. found similar results, in that women with HIV infection had 0.93 times lower serum ferritin concentrations compared to women not infected with HIV (95\% CI: 0.86, 0.99) (Friis et al., 2001).

An interesting finding in the present study was that hemoglobin and ferritin concentrations were significantly and inversely correlated, at both baseline and week 10, even after adjusting for CRP concentration and HIV status. The incongruity between ferritin and hemoglobin concentrations may be due to the serum analysis of ferritin versus capillary analysis of hemoglobin. The study by Friis et al. (2001) found conflicting results; low serum ferritin was a significant predictor of low hemoglobin in the 
Zimbabwean pregnant women (Friis et al., 2001). However, the study by Kaestel et al. (2015) found that pregnant women in Guinea-Bisseau with high serum ferritin concentrations $(>150 \mathrm{ug} / \mathrm{L})$ were significantly more likely to have lower hemoglobin concentrations after adjustment for the acute phase response. The authors indicate that this implies the acute phase response was not fully controlled for, which could be true for the present study as well (Kaestel et al., 2015).

Serum ferritin tends to normalize later than CRP does, thus has been suggested to use a "late" acute phase protein, such as alpha-1-acid glycoprotein (AGP) in addition to CRP, the "early" acute phase protein (Kaestel et al., 2015). The fact that infections and inflammation are so strongly related to ferritin may indicate that a second acute phase protein should be assessed in future research to gain a full picture of the inflammatory response. It is likely that the inverse correlation between ferritin and hemoglobin is partially due to inadequate adjustment of the inflammatory response.

Another interesting finding was that maternal baseline ferritin concentrations were significantly and negatively correlated to infant birth weight and length. A review by Allen (2000) states that a negative relationship between serum ferritin and birth weight is likely due to the presence of infection (Allen, 2000), however, in the present study, the significance remained even after controlling for CRP. This could be another indication that a second acute phase protein should be measured in studies of micronutrient assessment during pregnancy.

\subsection{Zinc}

Zinc deficiency at baseline in this population was high, at $38.2 \%, 29.0 \%$, and $38.8 \%$ in the CSB-IFA, CSB-UNIMMAP, and RUSF groups, respectively. Zinc 
concentrations decreased from baseline to week 10 in the CSB-IFA and CSB-UNIMMAP groups, however, zinc concentrations increased from week 0 to week 10 in the RUSF group, although this was not significant. The RUSF and CSB-UNIMMAP treatment foods had similar amounts of zinc, while the CSB-IFA treatment had less than half of that amount, but is still greater than the RDA for zinc during pregnancy.

Other research has found that maternal serum zinc concentrations decline during pregnancy (L. E. Caulfield et al., 1999). Although it was not significant, it is interesting that the mean zinc concentration of the RUSF group increased throughout the study, while the other groups experienced the traditional declines. Caulfield et al. (1999) found that Peruvian pregnant women supplemented with iron, folic acid and zinc had significantly higher serum zinc concentrations compared to women supplemented with iron and folic acid alone (L. E. Caulfield et al., 1999). However, this is different from the present study because our control group (CSB-IFA) still received $11 \mathrm{mg}$ of zinc in their supplemental food.

In this population, gestational age was significantly associated with baseline zinc concentration. As is consistent with the literature, women who were further along in their pregnancy had significantly lower baseline zinc concentrations. Zinc status at either time point was not significantly related to parity, which differs from other studies. A study in rural Malawian pregnant women found that primiparous women had significantly higher hair zinc concentrations (but not plasma zinc) compared to multiparous women. Hair zinc is used to assess chronic zinc status over time (Gibson \& Huddle, 1998).

Zinc concentrations were significantly and positively related to both ferritin and hemoglobin concentrations at baseline, and significantly related to hemoglobin at week 
10. The association between zinc and iron status has been reported in the literature; however, research has shown that zinc and iron tend to be negatively correlated. Iron and zinc compete for binding sites in the body, thus supplementation with both may affect its efficacy on serum status of zinc or iron markers (Nguyen et al., 2012). A study in nonpregnant Guatemalan women found that there were no differences in iron or zinc status after supplementation for 12 weeks with IFA, with or without zinc (Nguyen et al., 2012). Similarly, a study in pregnant Peruvian women did not find any differences in serum ferritin or hemoglobin concentrations after supplementing with IFA with or without zinc (Zavaleta, Caulfield, \& Garcia, 2000). In contrast, Ghanaian pregnant iron-deficient women receiving a combination of IFA and zinc had improved hemoglobin and ferritin concentrations compared to IFA supplements alone (Saaka, 2012).

Dietary zinc intake in Malawian women has been reported as inadequate (Gibson $\&$ Huddle, 1998). Cereals and grains are staples in Malawian diet, which are both low in zinc and high in phytates, which are known to inhibit zinc absorption. Phytates are also found in corn, which is the basis of the CSB supplementary food. Readily available zinc is found in animal products, which are not often consumed in low-income or rural Malawians. A study of dietary intake in rural Malawian pregnant women found that the median intake of zinc was $9.0 \mathrm{mg}$ per day, of which only $20.6 \%$ was available (as estimated by a ratio of phytate to zinc). The women in that study who had higher dietary phytate-zinc ratios had significantly lower hair zinc concentrations (Gibson \& Huddle, 1998). A higher phytate diet is likely to result in low zinc concentrations, which could be a factor in the present study relating to the high rates of zinc deficiency. 
Studies have generally found that the addition of zinc to a prenatal supplement (usually containing iron and folic acid, with or without additional micronutrients) does not have an effect on birth outcomes or measurements. The study with Peruvian pregnant women mentioned above also analyzed birth outcomes after supplementing with IFA, with or without zinc (L. Caulfield, Zavaleta, Figueroa, \& Leon, 1999). Data from 957 infants found no differences in birth weight, head circumference, MUAC, or incidence of low birth weight or preterm birth (L. Caulfield et al., 1999). In Nepal, pregnant women receiving iron, folic acid and zinc supplements did not have infants with greater birth weight, length or head circumferences compared to infants of women receiving one of four other treatments (folic acid, folic acid and iron, multiple micronutrients, or vitamin A alone) (Christian et al., 2003). The present study did not have a control group without zinc; therefore it is unknown whether prenatal supplementation with zinc has an effect on maternal zinc concentrations over iron and folic acid alone. The present study was also inadequately powered to detect differences in infant birth measurements.

Due to the fact that there were no significant differences between maternal serum zinc concentrations at week 10, there may not be a need for double the RDA of zinc during prenatal supplementation. At week 10, approximately one-third of women were still zinc deficient, which suggests that there are other factors contributing to zinc deficiency in addition to inadequate intake and low bioavailability due to anti-nutrients like phytate.

\subsection{Hemoglobin}

Change in capillary hemoglobin concentrations did not differ significantly by treatment group. Seventy-one percent of the women were anemic at baseline with 
hemoglobin concentrations $<11 \mathrm{~g} / \mathrm{dL}$, which was improved to $45 \%$ at week 10 .

Hemoglobin was slightly improved from week 0 to week 10 in all groups, but not significantly. The overall mean improved from $10.07 \mathrm{~g} / \mathrm{dL}$ at week 0 to $11.12 \mathrm{~g} / \mathrm{dL}$ at week 10 .

Women with higher parity had greater baseline hemoglobin concentrations. In contrast, it has been reported previously that women with higher parity are more likely to have lower iron markers, due to nutritional depletion and iron loss during multiple pregnancies (Scholl, 2005). A potential reason for the present results could be that women who had previous pregnancies were taking iron supplements and built up their iron stores. Women with lower gestational age had greater hemoglobin concentrations, which could be because they had not yet transferred as much of their iron stores to the fetus compared to women who were further along in their pregnancies.

Hemoglobin and albumin were significantly correlated. Of anemic women at baseline, $48.2 \%$ also had low serum albumin, while $28.4 \%$ of non-anemic women were low. This could be due to the low dietary intake of animal products, which are good sources of both iron and protein. A study in North Indian pregnant women found that those who were anemic and primiparous had significantly lower albumin concentrations compared to non-anemic, multiparous women (H. Shankar et al., 2015).

Clinic site was also related to hemoglobin. Women living in the Zomba district had significantly higher baseline hemoglobin concentrations, compared to women in the Mulanje, Chikhwawa, and Blantyre districts. The difference in hemoglobin concentrations may be due to diet variation or perhaps differences in infections or parasites that can be related to anemia. 
In the present study, treatment group had no effect on hemoglobin concentrations. In contrast, a study by Adu-Afarwuah et al. (2016) randomized Ghanaian women to receive iron and folic acid (60 mg Fe), MMN (20 mg Fe), or LNS (20 mg Fe). At week 36 of gestation, the IFA group had significantly greater hemoglobin concentrations and lower prevalence of anemia when compared to the MMN or LNS groups (Adu-Afarwuah et al., 2016). These results would be expected in the present study because of the greater amount of iron in the CSB-IFA treatment food compared to CSB-UNIMMAP and RUSF; however, no differences were found.

The lack of differences in anemia rates between treatment groups in the present study could be partially due to anemia having multiple causes. If iron deficiency were the only cause of anemia, then it would be logical that the majority of women receiving iron supplementation would recover from anemia. However, all women in this study received almost double or triple the RDA for iron; thus, it is likely that there are other factors contributing to anemia in this population, such as protein-energy malnutrition, malaria, parasites and other infections.

\subsection{Albumin}

Prevalence of hypoalbuminemia was high in this population and concentrations decreased in all groups from week 0 to week 10. Overall hypoalbuminemia rates were $42.7 \%$ at baseline and $70.1 \%$ at week 10 . Because the mean albumin concentrations at baseline were close to the deficiency cut-off value of $3.4 \mathrm{~g} / \mathrm{dL}$, the slight decrease that each group experienced resulted in a much larger proportion of deficiency at week 10.

Albumin is a negative acute phase protein, thus it is expected to decrease in the presence of inflammation (Tomkins, 2003). This was true for baseline measures, in which 
albumin and CRP were negatively correlated. However, the significance of this association was lost when measuring week 10 concentrations, and did not differ by treatment groups. HIV-positive and HIV-negative women had similar albumin concentrations. This differs from a study that found lactating women in South Africa who were HIV-infected had lower serum albumin concentrations compared to HIV-uninfected women after controlling for the acute phase response (Papathakis, Rollins, Chantry, Bennish, \& Brown, 2007). This study also found that serum albumin increased in all women from 6 weeks to 24 weeks postpartum (Papathakis et al., 2007). Thus, it is possible that women in the present study experienced decreases in albumin concentrations due to inadequate protein intake as well as pregnancy itself being an inflammatory process.

\subsection{C-reactive protein}

C-reactive protein (CRP) concentrations were highly elevated in this population with over half of the women experiencing elevated CRP at baseline. The mean CRP concentrations increased from baseline to week 10 in all groups, although the percentage of women with elevated status decreased, likely due to the large standard deviation in concentrations.

In this population, malarial infection and HIV infection were significantly related to CRP concentration, as is expected with the inflammatory response. Women who had HIV or malaria had significantly greater CRP concentrations than women not infected with either disease. In the study by Papathakis et al. (2007), HIV-infected lactating women had higher CRP concentrations compared to HIV-uninfected women, although the differences were not significant (Papathakis et al., 2007). A study in Ghanian 
pregnant women found that mean CRP concentrations were 5.8-7.9 mg/L (Adu-Afarwuah et al., 2016), which is markedly lower than overall baseline CRP mean of $11.3 \mathrm{mg} / \mathrm{L}$ in the present study.

Treatment group had no significant effect on CRP concentrations in this study. The study by Adu-Afarwuah et al. (2016) described previously found similar results. All groups (IFA, MMN, and LNS) in that study saw a small decrease in mean CRP concentrations after receiving one of the three interventions during pregnancy; however, none of the treatment groups improved inflammatory status better than another (measured by both CRP and AGP) (Adu-Afarwuah et al., 2016). A study in Nepal randomized women to receive IFA or MMN supplementation during pregnancy and collected blood samples at baseline (12-20 weeks gestation) and at week 32 of gestation (Hindle et al., 2006). In the Nepalese population, mean CRP at week 36 of gestation was $1.33 \mathrm{mg} / \mathrm{L}$, which is much lower than the CRP found in the present study. CRP concentrations did not differ significantly between the MMN and IFA groups, which is consistent with the present study (Hindle et al., 2006).

In the present study, there was a trend of higher maternal CRP at week 10 and lower infant birth MUAC ( $\mathrm{p}=0.056)$. No other birth measurements were significantly related to maternal CRP status. The study by Hindle et al. (2006) found that women with higher inflammatory markers were more likely to have infants with preterm birth (elevated CRP and AGP) and low birth weight (elevated AGP) (Hindle et al., 2006). Although the present study did not measure preterm birth, rates of low birth weight were not affected by CRP concentrations. Hindle et al. suggest that the relationship between 
elevated CRP and preterm birth may be related to the relationship between preterm birth and reproductive tract infections (Hindle et al., 2006).

Overall, CRP concentrations in this population were highly elevated, even in the absence of HIV infection or self-reported malaria, pneumonia, or diarrhea within the previous two months. The disparity between infection rates and elevated CRP indicate that there may be other underlying causes of an inflammatory state. The methods of determining illness data for this study were not medical diagnoses but were self-reported; therefore it is possible that women did not correctly recall their recent illnesses. Also, there could be other illnesses not asked on the baseline questionnaire that could be contributing to elevated CRP. For example, parasitic infections may be a factor affecting this population. Lastly, CRP may be elevated more in this population due to the inflammatory state of pregnancy itself and the fact that this study only included moderately malnourished women. Women who are more adequately nourished are better able to fight off infections and illnesses (Lartey, 2008).

\subsection{Birth and infant measurements}

Some trends between maternal nutrient concentrations and infant measurements were found. At baseline, a lower maternal ferritin correlated to a higher infant birth weight. At three months, a lower maternal baseline ferritin then correlated to a lower infant weight. There has been concern about over-supplementation in women who are iron sufficient. A study in African-American pregnant women found that those in the highest quartile of plasma ferritin concentrations at week 26 of gestation had 2.7 times increased risk of preterm birth, 3.9 times increased risk of very low birth weight $(<1500$ g) and 2.0 times increased risk of LBW $(<2500 \mathrm{~g})$ infants, compared to women with 
ferritin concentrations in the lowest quartiles (Goldenberg et al., 1996). However, the researchers did not account for the acute phase response, which could affect both ferritin concentrations and adverse birth outcomes, thus it is difficult to generalize these findings. However, other research has found that high maternal iron status during pregnancy may lead to decreased fetal growth. High hemoglobin $(>12.0 \mathrm{~g} / \mathrm{dL})$ has been linked to LBW infants, as well as low hemoglobin $(<8.0 \mathrm{~g} / \mathrm{dL})$ (Scholl, 2005). In the United States, hemoglobin concentrations of $>14.4 \mathrm{~g} / \mathrm{dL}$ during the first and second trimesters have been associated with SGA infants (Scanlon et al., 2000). Changes in hemoglobin concentration during pregnancy typically occur due to changes in plasma volume and hemodilution (Steer, 2000). However, if the plasma volume does not expand enough, fetal growth may be restricted, leading to SGA or LBW infants, who are then more likely to be born preterm. The mechanism by which inadequate plasma volume expansion leads to restricted fetal growth may be related to changes in blood viscosity that reduces blood flow to and from the placenta (Steer, 2000).

In the present study, mean hemoglobin at baseline (typically in the first or second trimester) was $10.1 \mathrm{~g} / \mathrm{dL}$, with a range of 7.0-15.1 g/dL. Decreases in hemoglobin concentrations are expected throughout pregnancy; however, the present study saw the opposite. At week 10, the mean hemoglobin concentration increased by $1.0 \mathrm{~g} / \mathrm{dL}$. Maternal baseline and infant 3-month hemoglobin concentrations were significantly and positively correlated. The mean infant hemoglobin was $9.5 \mathrm{~g} / \mathrm{dL}$ (range $2.5-14.4 \mathrm{~g} / \mathrm{dL}$ ), which was not significantly different by treatment group.

Another interesting finding is that HIV-infected women were more likely to have infants with greater birth weights $(2.86 \mathrm{~kg})$, compared to HIV-uninfected women $(2.68$ 
$\mathrm{kg}$ ), when controlling for the effect of treatment. A study in Zimbabwe that randomized pregnant women to receive MMN or IFA supplements found no significant differences in infant birth weight between HIV-infected or HIV-uninfected women (Friis et al., 2004a). However, HIV-infected women receiving MMN supplements had a trend towards infants with greater birth weights (3017 g) compared to HIV-infected women receiving IFA supplements (2916 g) (95\% CI -3, 205; $\mathrm{p}=0.057)$. These authors had expected HIVinfected women to have infants with greater birth weights because they predicted MMN supplements would produce a larger effect and benefit to these women due to the increased nutrient requirements that occur with HIV (Friis et al., 2004a). Although there were no significant differences in infant size by treatment group for HIV-infected women in the present study, the reasoning provided by Friis et al. (2004a) is logical; HIVinfected women receiving nutritional interventions may respond more positively than women without disease.

\subsection{Strengths and limitations}

A major strength of this study was the fact that it was randomized. Assessment of serum nutrient status at two time points during the pregnancy was a strength that enabled us to evaluate change in nutrient status. The fact that the primary study (Mamachiponde) gathered so much baseline data and anthropometric measurements at each visit allowed this sub-study to utilize much more information.

Limitations include the inability to double-blind the study. Researchers were blinded; however, due to the nature of the interventions and their visible differences, it was impossible to blind the subjects. However, that did not likely affect the outcomes of the study. Loss to follow-up was high in this study ( $\mathrm{n}=112,33 \%)$, with the majority of the 
women lost because they delivered their infant before the second blood draw $(n=62)$. Due to the high rates of delivery before the second blood draw, the inclusion and exclusion criteria for this sub-study were altered partway through the study to require a lower gestational age, which could be considered a limitation. Another criterion that was altered after initiation of the primary study was the age criteria, which was lowered from 18 to 16 years. This should not have had an effect on outcomes. However, a factor that could affect outcomes is that compliance with the interventions was unknown for all subjects. It's also possible that the treatment foods were shared with other family members, even though the women were advised not to. However, due to the randomized nature of the study, the effect between groups should be similar.

There were some methodological drawbacks in this study. Due to women having to walk far distances and wait long periods of time for their antenatal visits, it seemed unethical and unfeasible to advise women to fast before their blood draws. Sixty-six percent of women were in a fasted state for the first blood draw, however, only 37 percent were fasted at the second blood draw. Fasting status did have an impact on some nutrients (zinc, folate, retinol), but this variable was accounted for in all analyses. Due to the long distance between some clinic sites and the laboratory, the range of time from blood draw to centrifuge was two to five hours. This could potentially have had an impact on nutrient assessment and outcome. Another limitation would be that only one marker of inflammation (CRP) was measured. It would be ideal to have two measures (i.e. CRP and AGP) to be able to assess both early and late stages of inflammation.

If there were unlimited resources, it would have been interesting to assess a full nutrient panel. Some micronutrients were present in the RUSF treatment food, but not in 
the CSB-IFA and/or CSB-UNIMMAP treatment, including copper, magnesium, and selenium. It would have been interesting to measure the serum status of those minerals. Also, even though the CSB-IFA group was considered the control group, the treatment food still contained calories, macronutrients, and micronutrients other than iron and folic acid, which could make some of the results difficult to interpret. However, because all of the women in the study were moderately malnourished, it would be unethical to have a true control group with no treatment. On a related note, because all the women were moderately malnourished, the results of this study may be not able to be generalized to the adequately nourished population of pregnant women. Finally, this sub-study did not have adequate power to detect differences in infant outcomes. However, the primary study (Mamachiponde) does have adequate power and will be assessing infant outcomes. 


\section{CHAPTER 6: CONCLUSIONS}

Multiple micronutrient (MMN) supplements or fortified food-based supplements may be an effective way to improve maternal nutrition, which in turn has the ability to improve infant and child health. Since micronutrient deficiencies can lead to adverse pregnancy and birth outcomes, it is imperative to figure out the ideal method to support maternal health, especially in developing countries like Malawi. This study adds evidence to the growing body of research.

It was hypothesized that supplementation with a micro- and macronutrient fortified supplementary food (RUSF) would improve the serum nutrient status of pregnant women more than supplementation with a fortified corn-soy blended flour combined with either iron and folic acid (CSB-IFA) or a multiple micronutrient supplement (CSB-UNIMMAP). However, the only nutrients that saw significant differences between treatment groups were vitamins $\mathrm{B}_{12}$ and $\mathrm{D}$, with RUSF providing the greatest improvement. For vitamin D status, there may have been a greater improvement in those receiving RUSF treatment because of the fatty acid content in that intervention, which could increase absorption of vitamin D. Vitamin D is a crucial nutrient for proper immune functioning of both mother and fetus and deficiency is associated with impaired fetal growth. Maternal deficiencies in vitamin $\mathrm{B}_{12}$ during pregnancy have been associated with adverse birth outcomes, including restricted fetal growth, stillbirth, or preterm birth, and increased risk of anemia and preeclampsia in the mother.

The high rates of deficient or marginal status in all nutrients and proteins are concerning and are similar to other studies of pregnant women in Malawi or other developing countries. The fact that supplementation with $200 \%$ the RDA of most 
micronutrients still did not improve nutrient status of all women indicates that other factors are altering the women's nutritional state, potentially including infections, inflammation, malabsorption, genetic differences, or nutrient interactions. It needs to be considered that iron and zinc have a competing relationship, and that high iron status has been linked to increased rates of infection, specifically malaria. Given that RUSF produced improved vitamin $\mathrm{D}$ and $\mathrm{B}_{12}$ status, it suggests that using a fortified supplementary food to improve the nutritional status of moderately malnourished pregnant women may be indicated. However, not all nutrients were significantly improved by the RUSF treatment, and folate concentrations were improved the most in the CSB-IFA group.

These results indicate that further research is needed. Micronutrients have a complex relationship, especially during pregnancy and in developing countries; thus, many additional and larger randomized controlled trials should be performed to lead international health policies in the right direction. Hopefully, future research can identify the best proportions of micro- and macronutrients to help pregnant women recover from malnutrition or nutrient deficiencies and improve pregnancy, birth, and infant outcomes. In conclusion, antenatal treatment of moderate malnutrition with a peanut-based, macroand micronutrient fortified supplementary food improved vitamins $\mathrm{B}_{12}$ and $\mathrm{D}$ concentrations more than treatment with fortified corn-soy blended flour plus iron and folic acid or a multiple micronutrient supplement. Ferritin, folate, retinol, zinc and albumin concentrations were not significantly different when comparing treatments. 


\section{REFERENCES}

Abbassi-Ghanavati, M., Greer, L., \& Cunninghahm, F. (2009). Pregnancy and laboratory studies: a reference table for clinicians. Obstetrics \& Gynecology, 114(6), 1326-31.

ACOG Committee on Obstetric Practice. (2011). ACOG Committee Opinion No. 495:

Vitamin D: Screening and supplementation during pregnancy. Obstetrics \& Gynecology, 118(1), 197-8.

Adair, L. S. (2014). Long-term consequences of nutrition and growth in early childhood and possible preventive interventions. Nestlé Nutrition Institute Workshop Series, 78, 111-20. doi:10.1159/000354949

Adu-Afarwuah, S., Lartey, A., Okronipa, H., Ashorn, P., \& Zeilani, M. (2016). Impact of small-quantity lipid-based nutrient supplement on hemoglobin, iron status and biomarkers of inflammation in pregnant Ghanaian women. Maternal and Child Nutrition. doi:10.1111/mcn.12262

Adu-Afarwuah, S., Lartey, A., Okronipa, H., Ashorn, P., Zeilani, M., Peerson, J. M., ... Dewey, K. G. (2015). Lipid-based nutrient supplement increases the birth size of infants of primiparous women in Ghana. The American Journal of Clinical Nutrition, 101, 835-846. doi:10.3945/ajcn.114.091546.Am

Aghajafari, F., Nagulesapillai, T., Ronksley, P. E., Tough, S. C., O’Beirne, M., \& Rabi, D. M. (2013). Association between maternal serum 25-hydroxyvitamin D level and pregnancy and neonatal outcomes: systematic review and meta-analysis of observational studies. BMJ (Clinical Research Ed.), 346(March), f1169. doi:10.1136/bmj.f1169

Ahmed, T., Hossain, M., \& Sanin, K. I. (2012). Global burden of maternal and child 
undernutrition and micronutrient deficiencies. Annals of Nutrition \& Metabolism, 61 Suppl 1(suppl 1), 8-17. doi:10.1159/000345165

Akhtar, S. (2013). Zinc status in South Asian populations--an update. Journal of Health, Population, and Nutrition, 31(2), 139-49. Retrieved from http://www.pubmedcentral.nih.gov/articlerender.fcgi?artid=3702335\&tool=pmcentr ez\&rendertype $=$ abstract

Allen, L. H. (2000). Anemia and iron deficiency: effects on pregnancy outcome. American Journal of Clinical Nutrition, 71(Suppl), 1280-1284.

Allen, L. H. (2005). Multiple micronutrients in pregnancy and lactation: an overview. The American Journal of Clinical Nutrition, 81(5), 1206S-1212S. Retrieved from http://www.ncbi.nlm.nih.gov/pubmed/15883453

Allen, L. H., Hampel, D., Shahab-ferdows, S., York, E. R., Adair, L. S., Flax, V. L., ... Bentley, M. E. (2015). Antiretroviral therapy provided to HIV-infected Malawian women in a randomized trial diminishes the positive effects of lipid-based nutrient supplements on breast-milk B vitamins. American Journal of Clinical Nutrition, 102, 1468-1474. doi:10.3945/ajen.114.105106.1

Arimond, M., Zeilani, M., Jungjohann, S., Brown, K. H., Ashorn, P., Allen, L. H., \& Dewey, K. G. (2013). Considerations in developing lipid-based nutrient supplements for prevention of undernutrition: experience from the International Lipid-Based Nutrient Supplements (iLiNS) Project. Maternal \& Child Nutrition, 1-31. doi:10.1111/men.12049

Ashorn, P., Alho, L., Ashorn, U., Cheung, Y. B., Dewey, K. G., Gondwe, A., ... Vosti, S. A. (2015). Supplementation of Maternal Diets during Pregnancy and for 6 Months 
Postpartum and Infant Diets Thereafter with Small-Quantity Lipid-Based Nutrient Supplements Does Not Promote Child Growth by 18 Months of Age in Rural Malawi : A Randomized Controlled Tri. The Journal of Nutrition, 145, 1345-1353. doi:10.3945/jn.114.207225.1345

Ashorn, P., Alho, L., Ashorn, U., Cheung, Y. B., Dewey, K. G., Harjunmaa, U., ... Maleta, K. (2015). The impact of lipid-based nutrient supplement provision to pregnant women on newborn size in rural Malawi: a randomized controlled trial. The American Journal of Clinical Nutrition, 101(2), 387-97. doi:10.3945/ajen.114.088617

Barrett, H., \& McElduff, A. (2010). Vitamin D and pregnancy: An old problem revisited. Best Practice \& Research. Clinical Endocrinology \& Metabolism, 24(4), 527-39. doi:10.1016/j.beem.2010.05.010

Baylin, A., Villamor, E., Rifai, N., Msamanga, G., \& Fawzi, W. W. (2005). Effect of vitamin supplementation to HIV-infected pregnant women on the micronutrient status of their infants. European Journal of Clinical Nutrition, 59(8), 960-8. doi:10.1038/sj.ejen.1602201

Beisel, W. (1996). Nutrition and immune function: Overview. The Journal of Nutritionutrition, 126, 2611S-2615S.

Bhate, V., Deshpande, S., Bhat, D., Joshi, N., \& Ladkat, R. (2009). Vitamin B12 status of pregnant Indian women and cognitive function in their 9-year-old children. Food and Nutrition Bulletin, 29(4), 249-254.

Bhutta, Z. a, \& Das, J. K. (2014). Interventions to address maternal and childhood undernutrition: current evidence. Nestlé Nutrition Institute Workshop Series, 78, 59_ 
69. doi:10.1159/000354941

Bhutta, Z. a, Das, J. K., Rizvi, A., Gaffey, M. F., Walker, N., Horton, S., ... Black, R. E. (2013). Evidence-based interventions for improvement of maternal and child nutrition: what can be done and at what cost? Lancet, 382(9890), 452-77. doi:10.1016/S0140-6736(13)60996-4

Bhutta, Z. a, Imdad, A., Ramakrishnan, U., \& Martorell, R. (2012). Is it time to replace iron folate supplements in pregnancy with multiple micronutrients? Paediatric and Perinatal Epidemiology, 26 Suppl 1, 27-35. doi:10.1111/j.1365-3016.2012.01313.x

Black, R. E. (2001). Micronutrients in pregnancy. British Journal of Nutrition, 85(S2), S193. doi:10.1079/BJN2000314

Black, R. E., Allen, L. H., Bhutta, Z. a., Caulfield, L. E., de Onis, M., Ezzati, M., ... Rivera, J. (2008). Maternal and child undernutrition: global and regional exposures and health consequences. The Lancet, 371(9608), 243-260. doi:10.1016/S01406736(07)61690-0

Bodnar, L., Rouse, D. J., Momirova, V., Peaceman, A. M., Sciscione, A., Spong, C. Y., ... Malone, F. D. (2013). Maternal 25-hydroxyvitamin D and preterm birth in twin gestations. Obstetrics \& Gynecology, 122(1), 91-98.

Bondevik, G., Schneede, J., Refsum, H., Lie, R., Ulstein, M., \& Kvale, G. (2001). Homocysteine and methylmalonic acid levels in pregnant Nepali women. Should cobalamin supplementation be considered? Eur J Clin Nutr, 55(10), 856-64.

Branca, F., Pastore, G., Demissie, T., \& Ferro-Luzzi, A. (1993). The nutritional impact of seasonality in children and adults of rural Ethiopia. European Journal of Clinical Nutrition, 47(12), 840-50. 
Brocklehurst, P., \& French, R. (1998). The association between maternal HIV infection and perinatal outcome : a systematic review of the literature and meta-analysis. British Journal of Obstetrics and Gynaecology, 105(August), 836-848.

Brough, L., Rees, G. a, Crawford, M. a, Morton, R. H., \& Dorman, E. K. (2010). Effect of multiple-micronutrient supplementation on maternal nutrient status, infant birth weight and gestational age at birth in a low-income, multi-ethnic population. The British Journal of Nutrition, 104(3), 437-45. doi:10.1017/S0007114510000747

Caulfield, L. E., Zavaleta, N., \& Figueroa, a. (1999). Adding zinc to prenatal iron and folate supplements improves maternal and neonatal zinc status in a Peruvian population. The American Journal of Clinical Nutrition, 69(6), 1257-63. Retrieved from http://www.ncbi.nlm.nih.gov/pubmed/10357748

Caulfield, L., Zavaleta, N., Figueroa, A., \& Leon, Z. (1999). Maternal Zinc Supplementation Does Not Affect Size at Birth or Pregnancy Duration in Peru. Journal of Nutrition, 129, 1563-1568.

Centers for Disease Control. (1992). Recommendations for the use of folic acid to reduce the number of cases of spina bifida and other neural tube defects. $M M W R, 41,1-7$. Central Intelligence Agency. (2014). The World Factbook: Malawi.

Checkley, W., West, K. P., Wise, R. a, Baldwin, M. R., Wu, L., LeClerq, S. C., ... Sommer, A. (2010). Maternal vitamin A supplementation and lung function in offspring. The New England Journal of Medicine, 362(19), 1784-94. doi:10.1056/NEJMoa0907441

Christakos, S., Hewison, M., Gardner, D. G., Wagner, C. L., Sergeev, I. N., Rutten, E., ... Bikle, D. D. (2013). Vitamin D: beyond bone. Annals of the New York Academy of 
Sciences, 1287, 45-58. doi:10.1111/nyas.12129

Christian, P., Khatry, S. K., Katz, J., Pradhan, E. K., Leclerq, S. C., Shrestha, S. R., ... Jr, K. P. W. (2003). Effects of alternative maternal micronutrient supplements on low birth weight in rural Nepal: double blind randomised community trial. British Medical Journal, 326, 1-6.

Christian, P., Manandhar, D. S., Khatry, S., Costello, A., \& West, K. P. (2005). Antenatal micronutrient supplements in Nepal. Lancet, 366, 711-12.

Christian, P., Schulze, K., Stoltzfus, R. J., \& Jr, K. P. W. (1998). Hyporetinolemia , illness symptoms, and acute phase protein response in pregnant women with and without night blindness. American Journal of Clinical Nutrition, 67, 1237-43.

Coutsoudis, A., Pillay, K., Spooner, E., Kuhn, L., \& Coovadia, H. (1999). Randomized trial testing the effect of vitamin A supplementation on pregnancy outcomes and early mother-to-child HIV-1 transmission in Durban, South Africa. South African Vitamin A Study Group. AIDS, 13(12), 1517-24.

Cox, S., Staalsoe, T., Arthur, P., Bulmer, J., Tagbor, H., Hviid, L., ... Kirkwood, B. (2005). Maternal vitamin A supplementation and immunity to malaria in pregnancy in Ghanaian primigravids. Tropical Medicine \& International Health, 10(12), 128697.

Danford, D. (1982). Pica and nutrition. Annu. Rev. Nutr., 2(303-22).

dePee, S., \& Dary, O. (2002). Biochemical Indicators of Vitamin A Deficiency : Serum Retinol and Serum Retinol Binding Protein. Journal of Nutrition, 132, 2895S$2901 \mathrm{~S}$.

Dey, A. C., Shahidullah, M., Mannan, M. A., Noor, M. K., Saha, L., \& Rahman, S. A. 
(2010). Maternal and Neonatal Serum Zinc Level and Its Relationship with Neural Tube Defects. J Health Popul Nutr, 28(4), 343-350.

Duggan, C., Srinivasan, K., Thomas, T., Samuel, T., Rajendran, R., Muthayya, S., ...

Kurpad, A. V. (2014). Vitamin B-12 Supplementation during Pregnancy and Early Lactation Increases Maternal, Breast Milk, and Infant Measures of Vitamin B-12. Journal of Nutrition, 144, 758-764. doi:10.3945/jn.113.187278.pregnancy-induced Ekvall, H. (2003). Malaria and anemia. Curr Opin Hematol, 10(2), 108-14.

Emmett, S. D., \& West, K. P. (2014). Gestational vitamin A deficiency: A novel cause of sensorineural hearing loss in the developing world? Med Hypotheses, 82(1), 6-10. doi:10.1016/j.mehy.2013.09.028.Gestational

Engle-Stone, R., Nankap, M., Ndjebayi, A. O., Erhardt, J. G., \& Brown, K. H. (2013). Plasma Ferritin and Soluble Transferrin Receptor Concentrations and Body Iron Stores Identify Similar Risk Factors for Iron Deficiency but Result in Different Estimates of the National Prevalence of Iron Deficiency and Iron-Deficiency Anemia among Women a. Journal of Nutrition, 143, 369-377. doi:10.3945/jn.112.167775.iron

FAO, \& WHO. (1998). Human Vitamin and Mineral Requirements.

Fawzi, W. W., Msamanga, G. I., Spiegelman, D., Urassa, E. J. N., Mcgrath, N., Mwakagile, D., ... Hunter, D. J. (1998). Randomised trial of effects of vitamin supplements on pregnancy outcomes and T cell counts in HIV-1-infected women in Tanzania. Lancet, 351, 1477-1482.

Fawzi, W. W., Msamanga, G. I., Spiegelman, D., Wei, R., Kapiga, S., Villamor, E., ... Hunter, D. J. (2004). A randomized trial of multivitamin supplements and HIV 
disease progression and mortality. The New England Journal of Medicine, 351(1), 23-32. doi:10.1056/NEJMoa040541

Fawzi, W. W., Msamanga, G. I., Urassa, W., Hertzmark, E., Petraro, P., Willett, W. C., \& Spiegelman, D. (2007). Vitamins and perinatal outcomes among HIV-negative women in Tanzania. The New England Journal of Medicine, 356(14), 1423-31. doi:10.1056/NEJMoa064868

Filteau, S., \& Tomkins, A. (1994). Micronutrients and tropical infections. Trans $R$ Soc Trop Med Hyg, 88, 1-3,26.

Food and Nutrition Board. (1998). Dietary Reference Intakes for Thiamin, Riboflavin, Niacin, Vitamin B6, Folate, Vitamin B12, Pantothenic Acid, Biotin, and Choline.

Food and Nutrition Board. (2001). Dietary Reference Intakes.

Fraker, P. (1994). Nutritional immunology: methodological considerations. J Nutr Immunol, 2, 87-92.

Friis, H. (2002). Micronutrients and HIV Infection.

Friis, H. (2006). Micronutrient interventions and HIV infection: a review of current evidence. Tropical Medicine \& International Health : TM \& IH, 11(12), 1849-57. doi:10.1111/j.1365-3156.2006.01740.x

Friis, H., Gomo, E., Ndhlovu, P., Nyazema, N., Krarup, H., \& Michaelsen, K. F. (2001). HIV and other predictors of serum folate, serum ferritin, and hemoglobin in pregnancy : a cross-sectional study in Zimbabwe. American Journal of Clinical Nutrition, 73, 1066-1073.

Friis, H., Gomo, E., Nyazema, N., Ndhlovu, P., Krarup, H., Kaestel, P., \& Michaelsen, K. F. (2004a). Effect of multimicronutrient supplementation on gestational length and 
birth size: a randomized, placebo-controlled, double-blind effectiveness trial in Zimbabwe. The American Journal of Clinical Nutrition, 80(1), 178-84. Retrieved from http://www.ncbi.nlm.nih.gov/pubmed/15213046

Friis, H., Gomo, E., Nyazema, N., Ndhlovu, P., Krarup, H., Kaestel, P., \& Michaelsen, K. F. (2004b). Maternal body composition, HIV infection and other predictors of gestation length and birth size in Zimbabwe. British Journal of Nutrition, 92(5), $833-40$.

Gay, C., Mwapasa, V., Murdoch, D. M., Kwiek, J. J., Fiscus, S. A., \& Meshnick, S. R. (2011). Acute HIV infection among pregnant women in Malawi. Diagn Microbiol Infect Dis, 66(4), 356-360. doi:10.1016/j.diagmicrobio.2009.12.001.Acute

Gebreegziabher, T., \& Stoecker, B. (2013). Vitamin D insufficiency in a sunshinesufficient area: Southern Ethiopia. Food and Nutrition Bulletin, 34(4), 429-33.

Georgieff, M. K. (2007). Nutrition and the developing brain: nutrient priorities and measurement. American Journal of Clinical Nutrition, 85(Suppl), 614-620.

Gibson, R. S., \& Huddle, J. M. (1998). Suboptimal zinc status in pregnant Malawian women: Its association with low intakes of poorly available zinc, frequent reproductive cycling, and malaria. American Journal of Clinical Nutrition, 67(4), 702-709.

Goldenberg, R. L., Tamura, T., DuBard, M., Johnston, K., Copper, R., \& Neggers, Y. (1996). Plasma ferritin and pregnancy outcome. American Journal of Obstetrics and Gynecology, 175(5), 1356-9.

Gopalan, C. (2002). Multiple micronutrient supplementation in pregnancy. Nutr Rev, $60(5(2)), \mathrm{S} 2-6$. 
Green, H., Pindar, D., Davis, G., \& Mellanby, E. (1931). Diet as a prophylactic agent against puerperal sepsis. British Medical Journal, 2(3691), 595-598.

Gropper, S. S., Smith, J. L., \& Groff, J. L. (2009). Advanced Nutrition and Human Metabolism. Advanced Nutrition and Human Metabolism.

Gropper, S., Smith, J., \& Groff, J. L. (2009). The Water-Soluble Vitamins. In Advanced Nutrition and Human Metabolism (pp. 358-363).

Guyatt, H., \& Snow, R. (2001). The epidemiology and burden of Plasmodium falciparum-related anemia among pregnant women in sub-Saharan Africa. Am J Trop Med Hyg, 64(1-2 Suppl), 36-44.

Guyatt, H., \& Snow, R. (2004). Impact of malaria during pregnancy on low birth weight in sub-Saharan Africa. Clinical Microbiology Reviews, 17(4), 760-9. doi:10.1128/CMR.17.4.760

Haider, B., \& Bhutta, Z. (2012). Multiple-micronutrient supplementation for women during pregnancy ( Review ). Cochrane Database of Systematic Reviews, (11).

Haider, B., \& Bhutta, Z. (2015). Multiple-micronutrient supplementation for women during pregnancy (Review). Cochrane Database of Systematic Reviews, (11).

Hallberg, L., Hulten, L., \& Gramatkovski, E. (1997). Iron absorption from the whole diet in men: how effective is the regulation of iron absorption? American Journal of Clinical Nutrition, 66, 347-56.

Higashi, A., Tajiri, A., Matsukura, M., \& Matsuda, I. (1988). A prospective survey of serial maternal serum zinc levels and pregnancy outcome. J Pediatr Gastroenterol Nutr, 7(3), 430-3.

Hindle, L. J., Gitau, R., Filteau, S. M., Newens, K. J., Osrin, D., \& Costello, A. M. 
(2006). Effect of multiple micronutrient supplementation during pregnancy on inflammatory markers in Nepalese women. American Journal of Clinical Nutrition, 84, 1086-1092.

Hine, R. (1993). Folic acid: Contemporary clinical perspective. Perspect. Appl. Nutr, 1, $3-14$.

Hotz, C., Peerson, J. M., \& Brown, K. H. (2003). Suggested lower cutoffs of serum zinc concentrations for assessing zinc status: reanalysis of the second National Health and Nutrition Examination Survey data (1976-1980). American Journal of Clinical Nutrition, 78, 756-64.

Hovdenak, N., \& Haram, K. (2012). Influence of mineral and vitamin supplements on pregnancy outcome. European Journal of Obstetrics, Gynecology, and Reproductive Biology, 164(2), 127-32. doi:10.1016/j.ejogrb.2012.06.020

Huffman, S. L., Baker, J., Shumann, J., \& Zehner, E. (1998). The Case for Promoting Multiple Vitamin/Mineral Supplements for Women of Reproductive Age in Developing Countries.

Hult, M., Tornhammar, P., Ueda, P., Chima, C., Bonamy, A. E., Ozumba, B., \& Norman, M. (2010). Hypertension, diabetes and overweight: looming legacies of the Biafran famine. PloS One, 5(10), 1-8. doi:10.1371/journal.pone.0013582

Huybregts, L., Roberfroid, D., Lanou, H., Menten, J., Meda, N., \& Camp, J. Van. (2009). Prenatal food supplementation fortified with multiple micronutrients increases birth length : a randomized controlled trial in rural Burkina. American Journal of Clinical Nutrition, 90, 1593-600. doi:10.3945/ajen.2009.28253.Am

Imdad, A., \& Bhutta, Z. (2012a). Effects of calcium supplementation during pregnancy 
on maternal, fetal and birth outcomes. Paediatric and Perinatal Epidemiology, 26(SUPPL 1), 138-52.

Imdad, A., \& Bhutta, Z. A. (2012b). Routine iron/folate supplementation during pregnancy: Effect on maternal anaemia and birth outcomes. Paediatric and Perinatal Epidemiology, 26(SUPPL. 1), 168-177. doi:10.1111/j.13653016.2012.01312.x

Janmohamed, A., Karakochuk, C. D., Boungnasiri, S., Chapman, G. E., Janssen, P. A., Brant, R., ... Mclean, J. (2016). Prenatal supplementation with Corn Soya Blend Plus reduces the risk of maternal anemia in late gestation and lowers the rate of preterm birth but does not significantly improve maternal weight gain and birth anthropometric measurements in rural Cambodian. American Journal of Clinical Nutrition, 103, 559-66. doi:10.3945/ajcn.114.104034.1

Joy, E. J. M., Ander, E. L., Young, S. D., Black, C. R., Watts, M. J., Chilimba, A. D. C., ... Broadley, M. R. (2014). Dietary mineral supplies in Africa. Physiologia Plantarum, 151(3), 208-29. doi:10.1111/ppl.12144

Kaestel, P., Aaby, P., Ritz, C., \& Friis, H. (2015). Markers of iron status are associated with stage of pregnancy and acute-phase response, but not parity among pregnant women in Guinea-Bissau. British Journal of Nutrition, 114, 1072-9. doi: $10.1017 /$ S0007114515001993

Kalra, P., Das, V., Agarwal, A., Kumar, M., Ramesh, V., Bhatia, E., ... Bhatia, V. (2012). Effect of vitamin D supplementation during pregnancy on neonatal mineral homeostasis and anthropometry of the newborn and infant. The British Journal of Nutrition, 108(6), 1052-8. doi:10.1017/S0007114511006246 
Karim, E., \& Mascie-Taylor, C. (1997). The association between birthweight, sociodemographic variables and maternal anthropometry in an urban sample from Dhaka, Bangaladesh. Annals of Human Biology, 24(5), 387-401.

Kawai, K., Spiegelman, D., Shankar, A. H., \& Fawzi, W. W. (2011). Maternal multiple micronutrient supplementation and pregnancy outcomes in developing countries: meta-analysis and meta-regression. Bulletin of the World Health Organization, 89(6), 402-411B. doi:10.2471/BLT.10.083758

Kennedy, G., Nantel, G., \& Shetty, P. (2003). The scourge of "hidden hunger": global dimensions of micronutrient deficiencies. FNA/ANA, 32, 8-16.

Kerac, M., Postels, D. G., Mallewa, M., Alusine Jalloh, A., Voskuijl, W. P., Groce, N., ... Molyneux, E. (2014). The interaction of malnutrition and neurologic disability in Africa. Seminars in Pediatric Neurology, 21(1), 42-9.

doi:10.1016/j.spen.2014.01.003

King, J. C. (2000). Determinants of maternal zinc status during pregnancy. The American Journal of Clinical Nutrition, 71(5 Suppl), 1334S-43S. Retrieved from http://www.ncbi.nlm.nih.gov/pubmed/10799411

Kirkwood, B., Hurt, L., Amenga-Etego, S., Tawiah, C., Zandoh, C., Danso, S., ... Arthur, P. (2010). Effect of vitamin A supplementation in women of reproductive age on maternal survival in Ghana (ObaapaVitA): a cluster-randomised, placebo-controlled trial. Lancet, 375(9726), 1640-9.

Klemm, R., Sommerfelt, A., Boyo, A., \& Barba, C. (2011). Are we making progress on reducing anemia in women? Cross-country Comparison Of Anemia Prevalence, Reach, and Use of Antenatal Care and Anemia Reduction Interventions. AED. 
Retrieved from

http://scholar.google.com/scholar?hl=en\&btnG=Search\&q=intitle:Are+We+Making +Progress+on+Reducing+Anemia+in+Women+?\#1

Klevor, M. K., Haskell, M. J., Lartey, A., Adu-afarwuah, S., Zeilani, M., \& Dewey, K. G. (2016). Lipid-Based Nutrient Supplements Providing Approximately the Recommended Daily Intake of Vitamin A Do Not Increase Breast Milk Retinol Concentrations among Ghanaian Women. Journal of Nutrition, 146, 335-342. doi:10.3945/jn.115.217786.335

Koj, A. (1985). Biological functions of acute phase proteins. In The Acute Phase Response to Injury and Infection (pp. 145-160).

Kramer, M. S., \& Kakuma, R. (2010). Energy and protein intake in pregnancy. Cochrane Database of Systematic Reviews, (4), CD000032. doi:10.1002/14651858.CD000032

Kumwenda, N., Miotti, P. G., Taha, T. E., Broadhead, R., Biggar, R. J., Jackson, J. B., ... Semba, R. (2002). Antenatal vitamin A supplementation increases birth weight and decreases anemia among infants born to human immunodeficiency virus-infected women in Malawi. Clinical Infectious Diseases, 35(5), 618-24. doi:10.1086/342297

Lartey, A. (2008). Maternal and child nutrition in Sub-Saharan Africa: challenges and interventions. The Proceedings of the Nutrition Society, 67(1), 105-8. doi: $10.1017 / \mathrm{S} 0029665108006083$

Liu, J., Mei, Z., Ye, R., Serdula, M. K., Ren, A., \& Cogswell, M. E. (2013). Micronutrient supplementation and pregnancy outcomes: double-blind randomized controlled trial in China. JAMA Internal Medicine, 173(4), 276-82. doi:10.1001/jamainternmed.2013.1632 
Lonn, E., Yusuf, S., Pogue, J., Micks, M., Mcqueen, M. J., Probstfield, J., ... Genest, J. (2006). Homocysteine Lowering with Folic Acid and B Vitamins in Vascular Disease. The New England Journal of Medicine, 354, 1567-1577.

Macallan, D. C. (1999a). Nutrition and immune function in human immunodeficiency virus infection. Proceedings of the Nutrition Society, 58, 743-748.

Macallan, D. C. (1999b). Wasting in HIV Infection and AIDS. Journal of Nutrition, 129(Suppl), 238-242.

Mahomed, K. (2007). Iron and folate supplementation in pregnancy. Cochrane Database of Systematic Reviews, 18(3), 1-135.

Makola, D., Ash, D. M., Tatala, S. R., Latham, M. C., \& Ndossi, G. (2003). Community and International Nutrition A Micronutrient-Fortified Beverage Prevents Iron Deficiency, Reduces Anemia and Improves the Hemoglobin Concentration of Pregnant Tanzanian Women. Community and International Nutrition, 133, 13391346.

Makrides, M., Anderson, A., Gibson, R. a, \& Collins, C. T. (2013). Improving the neurodevelopmental outcomes of low-birthweight infants. Nestlé Nutrition Institute Workshop Series, 74, 211-21. doi:10.1159/000348775

Manary, M. J. (2005). Local production and provision of ready-to-use therapeutic food for the treatment of severe childhood malnutrition, (November).

Mastala, Y., Nyangulu, P., Banda, R. V, Mhemedi, B., White, S. a, \& Allain, T. J. (2013). Vitamin D deficiency in medical patients at a central hospital in Malawi: a comparison with TB patients from a previous study. PloS One, 8(3), e59017. doi:10.1371/journal.pone.0059017 
McArdle, H. J., Gambling, L., \& Kennedy, C. (2014). Iron deficiency during pregnancy: the consequences for placental function and fetal outcome. The Proceedings of the Nutrition Society, 73(1), 9-15. doi:10.1017/S0029665113003637

McCauley, M., van den Broek, N. R., Dou, L., \& Othman, M. (2015). Vitamin A supplementation during pregnancy for maternal and newborn outcomes ( Review ). Cochrane Database of Systematic Reviews, (10).

Mcnulty, B., Mcnulty, H., Marshall, B., Ward, M., Molloy, A. M., Scott, J. M., ... Pentieva, K. (2013). Impact of continuing folic acid after the first trimester of pregnancy : findings of a randomized trial of Folic Acid Supplementation in the Second and Third Trimesters. American Journal of Clinical Nutrition, 98, 92-98. doi:10.3945/ajen.112.057489.1

McPartlin, J., Halligan, A., Scott, J., Darling, M., \& Weir, D. (1993). Accelerated folate breakdown in pregnancy. Lancet, 34(8838), 148-9.

Mehta, S., Giovannucci, E., Mugusi, F. M., Spiegelman, D., Aboud, S., Hertzmark, E., ... Fawzi, W. W. (2010). Vitamin D status of HIV-infected women and its association with HIV disease progression, anemia, and mortality. PloS One, 5(1), e8770. doi:10.1371/journal.pone. 0008770

Mehta, S., Mugusi, F. M., Spiegelman, D., Villamor, E., Finkelstein, J. L., Hertzmark, E., ... Fawzi, W. W. (2011). Vitamin D status and its association with morbidity including wasting and opportunistic illnesses in HIV-infected women in Tanzania. AIDS Patient Care and STDs, 25(10), 579-85. doi:10.1089/apc.2011.0182

Michelazzo, F. B., Oliveira, J. M., Stefanello, J., Luzia, L. a, \& Rondó, P. H. C. (2013). The influence of vitamin A supplementation on iron status. Nutrients, 5(11), 4399- 
413. doi:10.3390/nu5114399

Montgomery, K. S. (2003). Nutrition and HIV-Positive Pregnancy. Journal of Perinatal Education, 12(1), 42-47.

Muslimatun, S., Schmidt, M., Schultink, W., West, C., Hautvast, J., Gross, R., \& Muhilal. (2001). Weekly supplementation with iron and vitamin A during pregnancy increases hemoglobin concentration but decreases serum ferritin concentration in Indonesian pregnant women. Journal of Nutrition, 131(1), 85-90.

National Institutes of Health. (2014). Vitamin D Fact Sheet.

Ndekha, M. J., van Oosterhout, J. J. G., Zijlstra, E. E., Manary, M., Saloojee, H., \& Manary, M. J. (2009). Supplementary feeding with either ready-to-use fortified spread or corn-soy blend in wasted adults starting antiretroviral therapy in Malawi: randomised, investigator blinded, controlled trial. BMJ (Clinical Research Ed.), 338, b1867. doi:10.1136/bmj.b1867

Neggers, Y. H., Cutter, G. R., Acton, R. T., Alvarez, J. O., Bonner, J. L., Goldenberg, R. L., ... Roseman, J. M. (1990). A positive association between maternal serum zinc concentration and birth weight. American Journal of Clinical Nutrition, 51(4), 678684.

Nelms, M., Sucher, K. P., Lacey, K., \& Roth, S. L. (2007). Nutrition Therapy and Pathophysiology.

Neumann, C. G., Oace, S. M., Chaparro, M. P., Herman, D., Drorbaugh, N., \& Bwibo, N. O. (2014). Low vitamin B 12 intake during pregnancy and lactation and low breastmilk vitamin B 12 content in rural Kenyan women consuming predominantly maize diets. Food and Nutrition Bulletin, 34(2), 151-159. 
Nguyen, P., Grajeda, R., Melgar, P., Marcinkevage, J., Flores, R., Ramakrishnan, U., \& Martorell, R. (2012). Effect of Zinc on Efficacy of Iron Supplementation in Improving Iron and Zinc Status in Women Effect of Zinc on Efficacy of Iron Supplementation in Improving Iron and Zinc Status in Women. Journal of Nutrition and Metabolism, 1-8. doi:10.1155/2012/216179

Osrin, D., Vaidya, A., Shrestha, Y., Baniya, R. B., Manandhar, D. S., Adhikari, R. K., ... Costello, A. M. D. L. (2005). Effects of antenatal multiple micronutrient supplementation on birthweight and gestational duration in Nepal: double-blind, randomised controlled trial. Lancet, 365, 955-962.

Ouma, P., van Eijk, A. M., Hamel, M. J., Parise, M., Ayisi, J. G., Otieno, K., ... Slutsker, L. (2007). Malaria and anaemia among pregnant women at first antenatal clinic visit in Kisumu, western Kenya. Tropical Medicine \& International Health: TM \& IH, 12(12), 1515-23. doi:10.1111/j.1365-3156.2007.01960.x

Papathakis, P. C., Rollins, N. C., Chantry, C. J., Bennish, M. L., \& Brown, K. H. (2007). Micronutrient status during lactation in HIV-infected and HIV-uninfected South African women during the first 6 mo after delivery. The American Journal of Clinical Nutrition, 85(1), 182-92. Retrieved from http:/www.ncbi.nlm.nih.gov/pubmed/17209195

Pena-Rosas, J. P., De-Regil, L. M., Dowswell, T., \& Viteri, F. E. (2012). Daily oral iron supplementation during pregnancy. Cochrane Database of Systematic Reviewseviews, 12, 1-141. doi:10.1002/14651858.CD004736.pub4.Daily Peragallo Urrutia, R., \& Thorp, J. M. (2012). Vitamin D in Pregnancy: Current Concepts. Curr Opin Obstet Gynecol, 24(2), 57-64. 
doi:10.1097/GCO.0b013e3283505ab3.Vitamin

Persson, L. A., Arifeen, S., Ekstrom, E., Rasmussen, K. M., Frongillo, E. A., \& Yunus, M. (2012). Effects of Prenatal Micronutrient and Early Food Supplementation on Maternal Hemoglobin, Birth Weight, and Infant Mortality Among Children in Bangladesh. Journal of the American Medical Association, 307(19), 2050-2059.

Petraro, P., Duggan, C. P., Urassa, W., Msamanga, G. I., Makubi, A., Spiegelman, D., \& Fawzi, W. W. (2013). Determinants of anemia in postpartum HIV-negative women in Dar es Salaam, Tanzania. Eur J Clin Nutr, 67(7), 708-717. doi:10.1038/ejen.2013.71.Determinants

Pettifor, J. M., \& Prentice, A. (2011). The role of vitamin D in paediatric bone health. Best Practice \& Research. Clinical Endocrinology \& Metabolism, 25(4), 573-84. doi:10.1016/j.beem.2011.06.010

Prado, E. L., Alcock, K. J., Muadz, H., Ullman, M. T., \& Shankar, A. H. (2012). Maternal multiple micronutrient supplements and child cognition: a randomized trial in Indonesia. Pediatrics, 130(3), e536-46. doi:10.1542/peds.2012-0412

Prado, E. L., \& Dewey, K. G. (2014). Nutrition and brain development in early life. Nutrition Reviews, 72(4), 267-84. doi:10.1111/nure.12102

Prawirohartono, E., Nystrom, L., Nurdiati, D., Hakimi, M., \& Lind, T. (2013). The impact of prenatal vitamin A and zinc supplementation on birth size and neonatal survival-a double-blind, randomized controlled trial in a rural area of Indonesia. Int J Vitam Nutr Res, 83(1), 14-25.

President's Malaria Initiative. (2015). President's Malaria Initiative: Malawi.

Radhika, M., Bhaskaram, P., Balakrishna, N., \& Ramalakshmi, B. (2003). Red palm oil 
supplementation: a feasible diet-based approach to improve the vitamin A status of pregnant women and their infants. Food and Nutrition Bulletin, 24(2), 208-17.

Ramakrishnan, U. (2002). Prevalence of micronutrient malnutrition worldwide. Nutrition Reviews, 60(5 Pt 2), S46-52. Retrieved from http://www.ncbi.nlm.nih.gov/pubmed/12035858

Ramakrishnan, U., Imhoff-Kunsch, B., \& Martorell, R. (2014). Maternal nutrition interventions to improve maternal, newborn, and child health outcomes. Nestlé Nutrition Institute Workshop Series, 78, 71-80. doi:10.1159/000354942

Ramakrishnan, U., Manjrekar, R., Rivera, J., Gonzáles-Cossío, T., \& Martorell, R. (1999). Micronutrients and pregnancy outcome: A review of the literature. Nutrition Research, 19(1), 103-159. doi:10.1016/S0271-5317(98)00178-X

Ramlal, R., Tembo, M., King, C., Ellington, S., Soko, A., Chigwenembe, M., ... the BAN Study Team, E. (2015). Dietary Patterns and Maternal Anthropometry in HIVInfected, Pregnant Malawian Women. Nutrients, 7(1), 584-594. doi:10.3390/nu7010584

Rogerson, S. J., Mwapasa, V., \& Meshnick, S. R. (2007). Malaria in pregnancy: linking immunity and pathogenesis to prevention. The American Journal of Tropical Medicine and Hygiene, 77(6 Suppl), 14-22. Retrieved from http://www.ncbi.nlm.nih.gov/pubmed/18165470

Roseboom, T. J., Painter, R. C., van Abeelen, A. F. M., Veenendaal, M. V. E., \& de Rooij, S. R. (2011). Hungry in the womb: what are the consequences? Lessons from the Dutch famine. Maturitas, 70(2), 141-5. doi:10.1016/j.maturitas.2011.06.017

Roth, D. E., Perumal, N., Al Mahmud, A., \& Baqui, A. H. (2013). Maternal vitamin D3 
supplementation during the third trimester of pregnancy: effects on infant growth in a longitudinal follow-up study in Bangladesh. The Journal of Pediatrics, 163(6), 1605-1611.e3. doi:10.1016/j.jpeds.2013.07.030

Rush, D. (2000). Nutrition and maternal mortality in the developing world. The American Journal of Clinical Nutrition, 72(1 Suppl), 212S-240S.

Saaka, M. (2012). Combined iron and zinc supplementation improves haematologic status of pregnant women in Upper West Region of Ghana. Ghana Medical Journal, 46(4), 225-33. Retrieved from http://www.pubmedcentral.nih.gov/articlerender.fcgi?artid=3645177\&tool=pmcentr ez\&rendertype $=$ abstract

Sandstead, H. (1995). Requirements and toxicity of essential trace elements, illustrated by zinc and copper. American Journal of Clinical Nutrition, 61(3 Suppl), 621S$624 S$.

Sangaré, L., van Eijk, A. M., Ter Kuile, F. O., Walson, J., \& Stergachis, A. (2014). The association between malaria and iron status or supplementation in pregnancy: a systematic review and meta-analysis. PloS One, 9(2), e87743. doi:10.1371/journal.pone. 0087743

Sauberlich, H. (1985). Bioavailability of vitamins. Prog Food Nutr Sci, 9(1-2), 1-33.

Scanlon, K., Yip, R., Schieve, L., \& Cogswell, M. (2000). High and low hemoglobin levels during pregnancy: differential risks for preterm birth and small for gestational age. Obstetrics \& Gynecology, 96(5), 741-8.

Schantz-Dunn, J., \& Nour, N. (2009). Malaria and pregnancy: a global health perspective. Reviews in Obstetrics and Gynecology, 2(3), 186-192. 
doi:10.3909/riog0091

Schmidt, M. K., Muslimatun, S., West, C. E., Schultink, W., Gross, R., \& Hautvast, J. G. a J. (2002). Nutritional status and linear growth of Indonesian infants in West Java are determined more by prenatal environment than by postnatal factors. The Journal of Nutrition, 132(8), 2202-2207.

Scholl, T. O. (2005). Iron status during pregnancy: setting the stage for mother and infant. American Journal of Clinical Nutrition, 81, 1218-1222.

Scrimshaw, N. (1991). Iron deficiency. Scientific American, 265(4), 46-52.

Scrimshaw, N., \& Sangiovanni, J. (1997). Synergism of nutrition, infection, and immunity: an overview. American Journal of Clinical Nutrition, 66, 464S-77S.

Semba, R., Kumwenda, N., Taha, T., Mtimavalye, L., Broadhead, R., Garrett, E., ... Chiphangwi, J. D. (2001). Impact of vitamin A supplementation on anaemia and plasma erythropoietin concentrations in pregnant women: a controlled clinical trial. Eur J Haematol, 66(6), 389-95.

Semba, R., Miotti, P. G., Chiphangwi, J. D., Liomba, G., Yang, L. P., Saah, a J., ... Hoover, D. R. (1995). Infant mortality and maternal vitamin A deficiency during human immunodeficiency virus infection. Clinical Infectious Diseases, 21(4), 966972.

Semba, R., Miotti, P. G., Chiphangwi, J., Saah, A., Canner, J., Dallabetta, G., \& Hoover, D. (1994). Maternal vitamin A deficiency and mother-to-child transmission of HIV1. Lancet, 343(8913), 1593-7.

Senga, E. L., Harper, G., Koshy, G., Kazembe, P. N., \& Brabin, B. J. (2011). Reduced risk for placental malaria in iron deficient women. Malaria Journal, 10(1), 47. 
doi:10.1186/1475-2875-10-47

Shah, P. S., \& Ohlsson, A. (2009). Effects of prenatal multimicronutrient supplementation on pregnancy outcomes: a meta-analysis. CMAJ : Canadian Medical Association Journal = Journal de l'Association Medicale Canadienne, 180(12), E99-108. doi:10.1503/cmaj.081777

Shankar, A. H., Jahari, A., Sebayang, S., Aditiawarman, Apriatni, M., Harefa, B., \& Muadz, H. (2008). Effect of maternal multiple micronutrient supplementation on fetal loss and infant death in Indonesia: a double-blind cluster-randomised trial. Lancet, 371, 215-227.

Shankar, H., Kumar, N., Rao, D., Chandhiok, N., Sandhir, R., Kriplani, A., ... Kurra, S. (2015). Comparison of haematological and biochemical changes between nonanaemic and anaemic primigravid women in a north Indian population to establish normative values. Journal of Obstetrics and Gynaecoogy, 35, 221-224. doi:10.3109/01443615.2014.948817

Sommer, A., \& Davidson, F. R. (2002). Assessment and control of vitamin A deficiency: the Annecy Accords. The Journal of Nutrition, 132 (9 Suppl), 2845S-2850S.

Steer, P. J. (2000). Maternal hemoglobin concentration and birth weight. American Journal of Clinical Nutrition, 71(suppl), 1285-1287.

Steketee, R., Nahlen, B., Parise, M., \& Menendez, C. (2001). The burden of malaria in pregnancy in malaria-endemic areas. Am J Trop Med Hyg, 64(1-2 Suppl), 28-35.

Stephensen, C. B. (2001). Vitamin A, Infection, and Immune Function. Annu. Rev. Nutr., $21,167-92$.

Strand, T. A., Taneja, S., Ueland, P. M., Refsum, H., Bahl, R., Schneede, J., ... Bhandari, 
N. (2013). Cobalamin and folate status predicts mental development scores in North Indian children 12-18 mo of age. American Journal of Clinical Nutrition, 97, 310317. doi:10.3945/ajen.111.032268.INTRODUCTION

Suharno, D., West, C., Muhilal, Karyadi, D., \& Hautvast, J. (1993). Supplementation with vitamin A and iron for nutritional anaemia in pregnant women in West Java, Indonesia. Lancet, 342(8883), 1325-8.

Sukumar, N., Rafnsson, S. B., Kandala, N., Bhopal, R., Yajnik, C. S., \& Saravanan, P. (2016). Prevalence of vitamin B-12 insufficiency during pregnancy and its effect on offspring birth weight: a systematic review and. American Journal of Clinical Nutrition, 1-20. doi:10.3945/ajen.115.123083.

Swanson, C., \& King, J. C. (1987). Zinc and pregnancy outcome. American Journal of Clinical Nutrition, 46, 763-71.

Taggart, N. R., Holliday, R. M., Billewicz, W. Z., Hytten, F. E., \& Thomson, a M. (1967). Changes in skinfolds during pregnancy. The British Journal of Nutrition, 21(2), 439-451. doi:10.1079/BJN19670045

The Sphere Project. (2011). Humanitarian Charter and Minimum Standards in Humanitarian Response (Vol. 1). Retrieved from www.practicalactionpublishing.org/sphere

The World Bank. (2006). Repositioning Nutrition as Central to Development: A Strategy for Large Scale Action.

The World Bank. (2015). Malawi- Sub-Saharan Africa.

Thomas, R. M., Tembo, M., Soko, A., Chigwenembe, M., Tohill, B., Kayira, D., ... Der, C. Van. (2013). Patterns of body composition among HIV-infected, pregnant 
Malawians and the effects of famine season. Maternal and Child Nutrition, 17(2), 1-15. doi:10.1007/s10995-012-0970-6.Patterns

Thomas, R., Tembo, M., Soko, A., Chigwenembe, M., Ellington, S., Kayira, D., ...

Team, S. (2012). Maternal mid-upper arm circumference is associated with birth weight among HIV-infected Malawians. Nutr Clin Pract, 27(3), 416-421. doi:10.1177/0884533611435991.Maternal

Thorne-Lyman, A., \& Fawzi, W. W. (2012). Vitamin A and carotenoids during pregnancy and maternal, neonatal and infant health outcomes: A systematic review and meta-analysis. Paediatric and Perinatal Epidemiology, 29(6), 1-30. doi:10.1016/j.biotechadv.2011.08.021.Secreted

Thorne-Lyman, A., \& Fawzi, W. W. (2013). Vitamin D during pregnancy and maternal, neonatal and infant health outcomes: a systematic review and meta-analysis . Paediatric and Perinatal Epidemiology, 26(1), 2-3. doi:10.1111/j.13653016.2012.01283.x.Vitamin

Thurnham, D. I., \& Mccabe, G. P. (2012). Influence of infection and inflammation on biomarkers of nutritional status with an emphasis on vitamin A and iron.

Tomkins, A. (2003). Assessing micronutrient status in the presence of inflammation. The Journal of Nutrition, 133(5 Suppl 2), 1649S-1655S. Retrieved from http://www.ncbi.nlm.nih.gov/pubmed/12730480

Tsiaras, W. G., \& Weinstock, M. a. (2011). Factors influencing vitamin D status. Acta Dermato-Venereologica, 91(2), 115-124. doi:10.2340/00015555-0980

UNAIDS. (2012). Global Aids Response Progress Report: Malawi Country Report for 2010 and 2011. 
UNICEF. (2013). UNICEF: Malawi.

Uriu-Adams, J. Y., \& Keen, C. L. (2010). Zinc and reproduction: effects of zinc deficiency on prenatal and early postnatal development. Birth Defects Research. Part B, Developmental and Reproductive Toxicology, 89(4), 313-25. doi:10.1002/bdrb.20264

Uriu-Adams, J. Y., Obican, S. G., \& Keen, C. L. (2013). Vitamin D and maternal and child health: overview and implications for dietary requirements. Birth Defects Research. Part C, Embryo Today : Reviews, 99(1), 24-44. doi:10.1002/bdrc.21031 van den Broek, N. R., Dou, L., Othman, M., Neilson, J., Gates, S., \& Gulmezoglu, A. (2011). Vitamin A supplementation during pregnancy for maternal and newborn outcomes ( Review ). Cochrane Database of Systematic Reviews, (11).

van den Broek, N. R., \& Letsky, E. a. (2000). Etiology of anemia in pregnancy in south Malawi. The American Journal of Clinical Nutrition, 72(1 Suppl), 247S-256S. Retrieved from http://www.ncbi.nlm.nih.gov/pubmed/10871590

Verhoeff, F. H., Brabin, B. J., Buuren, S. Van, Chimsuku, L., Kazembe, P., Wit, J. M., \& Broadhead, R. L. (2001). An analysis of intra-uterine growth retardation in rural Malawi. European Journal of Clinical Nutrition, 55, 682-689.

Ververs, M., Antierens, A., Sackl, A., Staderini, N., \& Captier, V. (2013). Which anthropometric indicators identify a pregnant women as acutely malnourished and predict adverse birth outcomes in the humanitarian context? PLoS Current Disasters, 1, 1-19.

Victora, C. G., Adair, L. S., Fall, C., Hallal, P. C., Martorell, R., Richter, L., \& Sachdev, H. (2008). Maternal and child undernutrition: consequences for adult health and 
human capital. Lancet, 37(9609), 340-357.

Villamor, E., Saathoff, E., Manji, K., Msamanga, G., Hunter, D. J., \& Fawzi, W. W. (2005). Vitamin supplements, socioeconomic status, and morbidity events as predictors of wasting in HIV-infected women from Tanzania. The American Journal of Clinical Nutrition, 82(4), 857-65. Retrieved from http://www.ncbi.nlm.nih.gov/pubmed/16210717

Villamor, E., Saathoff, E., Msamanga, G. I., O’Brien, M., Manji, K. P., \& Fawzi, W. W. (2005). Wasting during pregnancy increases the risk of mother-to-child HIV-1 transmission. Journal of Acquired Immune Deficiency Syndromes, 38(5), 622-626.

Vollset, S. E., Refsum, H., Irgens, L. M., Emblem, B. M., Tverdal, A., Gjessing, H. K., ... Ueland, P. M. (2000). Plasma total homocysteine, pregnancy complications, and adverse pregnancy outcomes : the Hordaland Homocysteine Study $1-3$. American Journal of Clinical Nutrition, 71, 962-968.

Weir, D., \& Scott, J. (1999). Cobalamins Physiology, Dietary Sources and Requirements. In Encylopedia of Human Nutrition (pp. 394-401).

Weiss, G., \& Goodnough, L. T. (2005). Anemia of chronic disease. The New England Journal of Medicine, 352(10), 1011-23. doi:10.1056/NEJMra041809

West, K. P., Katz, J., Khatry, S., LeClerq, S. C., Pradhan, E., Shrestha, S., ... Sommer, A. (1999). Double blind, cluster randomised trial of low dose supplementation with vitamin A or beta carotene on mortality related to pregnancy in Nepal. The NNIPS-2 Study Group. British Medical Journal, 318(7183), 570-5.

West, K. P., Shamim, A. A., Mehra, S., Labrique, A. B., Ali, H., Shaikh, S., ... Christian, P. (2014). Effect of maternal multiple micronutrient vs iron-folic acid 
supplementation on infant mortality and adverse birth outcomes in rural Bangladesh: the JiVitA-3 randomized trial. Journal of the American Medical Association, 312(24), 2649-58. doi:10.1001/jama.2014.16819

Whittaker, P. (1998). Iron and zinc interactions in humans. American Journal of Clinical Nutrition, 68(2 SUPPL.), 442-446.

WHO. (1995). Physical Status: The Use and Interpretation of Anthropometry. Report of a WHO Expert Committee. Technical Report Series No. 854. doi:854

WHO. (2008). Conclusions of a WHO Technical Consultation on folate and vitamin B12 deficiencies. Food and Nutrition Bulletin, 29(2), 238-244.

WHO. (2009). Global prevalence of vitamin A deficiency in populations at risk 1995 2005.

WHO. (2012a). Guideline: Daily iron and folic acid supplementation in pregnant women. WHO. (2012b). Intermittent Preventive Treatment of malaria in pregnancy (IPTp) with Sulfadoxine-Pyrimethamine (SP).

WHO. (2012c). Serum and red blood cell folate concentrations for assessing folate status in populations.

WHO. (2014). Global Summary of the AIDS Epidemic.

WHO. (2015a). Maternal and Perinatal Health.

WHO. (2015b). Maternal Mortality Ratio.

World Food Programme. (2008a). Food Fortification in Malawi.

World Food Programme. (2008b). Malawi Nutrition: Therapeutic and Supplementary Feeding.

Yang, Z., \& Huffman, S. L. (2011). Review of fortified food and beverage products for 
pregnant and lactating women and their impact on nutritional status. Maternal \& Child Nutrition, 7 Suppl 3, 19-43. doi:10.1111/j.1740-8709.2011.00350.x

Young, S. L., Khalfan, S. S., Farag, T. H., Kavle, J. a, Ali, S. M., Hajji, H., ... Stoltzfus, R. J. (2010). Association of pica with anemia and gastrointestinal distress among pregnant women in Zanzibar, Tanzania. The American Journal of Tropical Medicine and Hygiene, 83(1), 144-51. doi:10.4269/ajtmh.2010.09-0442

Zavaleta, N., Caulfield, L. E., \& Garcia, T. (2000). Changes in iron status during pregnancy in Peruvian women receiving prenatal iron and folic acid supplements with or without zinc. American Journal of Clinical Nutrition, 71, 956-961.

Zeng, L., Dibley, M. J., Cheng, Y., Dang, S., Chang, S., Kong, L., \& Yan, H. (2008). Impact of micronutrient supplementation during pregnancy on birth weight, duration of gestation, and perinatal mortality in rural western China: double blind cluster randomised controlled trial. BMJ (Clinical Research Ed.), 337, a2001. doi:10.1136/bmj.a2001

Zhu, K., Whitehouse, A. J. O., Hart, P., Kusel, M., Mountain, J., Lye, S., ... Walsh, J. P. (2013). Maternal Vitamin D Status During Pregnancy and Bone Mass in Offspring at 20 Years of Age: A Prospective Cohort Study. Journal of Bone and Mineral Research : The Official Journal of the American Society for Bone and Mineral Research. doi:10.1002/jbmr.2138 


\section{APPENDICES}

APPENDIX A: RECOMMENDED DIETARY ALLOWANCES (RDA) OR ADEQUATE INTAKES (AI) FOR PREGNANCY ${ }^{1}$

\begin{tabular}{|c|c|c|c|}
\hline Vitamins & $<18$ years & $19-30$ years & $31-50$ years \\
\hline Thiamin (mg) & 1.4 & 1.4 & 1.4 \\
\hline Riboflavin (mg) & 1.4 & 1.4 & 1.4 \\
\hline Niacin (mg) & 18 & 18 & 18 \\
\hline Biotin (AI, mg) & 30 & 30 & 30 \\
\hline Pantothenic acid (AI, mg) & 6 & 6 & 6 \\
\hline Vitamin B6 (mg) & 1.9 & 1.9 & 1.9 \\
\hline Folate $(\mu \mathrm{g})$ & 600 & 600 & 600 \\
\hline $\operatorname{Vitamin}_{\mathrm{B}_{12}}(\mu \mathrm{g})$ & 2.6 & 2.6 & 2.6 \\
\hline Choline (AI, mg) & 450 & 450 & 450 \\
\hline Vitamin C (mg) & 80 & 85 & 85 \\
\hline Vitamin A $(\mu \mathrm{g})$ & 750 & 770 & 770 \\
\hline Vitamin D $(\mathrm{AI}, \mu \mathrm{g})^{2}$ & 15 & 15 & 15 \\
\hline Vitamin E (mg) & 15 & 15 & 15 \\
\hline Vitamin $\mathrm{K}(\mathrm{AI}, \mu \mathrm{g})$ & 75 & 90 & 90 \\
\hline \multicolumn{4}{|l|}{ Minerals } \\
\hline Sodium (AI, mg) & 1500 & 1500 & 1500 \\
\hline Chloride (AI, mg) & 2300 & 2300 & 2300 \\
\hline Potassium (AI, mg) & 4700 & 4700 & 4700 \\
\hline Calcium (AI, mg) & 1300 & 1000 & 1000 \\
\hline Phosphorus (mg) & 1250 & 700 & 700 \\
\hline Magnesium (mg) & 400 & 350 & 360 \\
\hline Iron (mg) & 27 & 27 & 27 \\
\hline Zinc (mg) & 12 & 11 & 11 \\
\hline Iodine $(\mu \mathrm{g})$ & 220 & 220 & 220 \\
\hline Selenium $(\mu \mathrm{g})$ & 60 & 60 & 60 \\
\hline Copper $(\mu \mathrm{g})$ & 1000 & 1000 & 1000 \\
\hline Manganese (AI, mg) & 2.0 & 2.0 & 2.0 \\
\hline Fluoride (AI, mg) & 3 & 3 & 3 \\
\hline Chromium (AI, $\mu \mathrm{g}$ ) & 29 & 30 & 30 \\
\hline Molybdenum $(\mu \mathrm{g})$ & 50 & 50 & 50 \\
\hline
\end{tabular}

'(S. S. Gropper et al., 2009)

${ }^{2}$ (National Institutes of Health, 2014) 


\section{Tolerable Upper Intake Levels (UL) for Pregnancy ${ }^{1}$}

\begin{tabular}{lll}
\hline Vitamins & $<18$ years & $19-50$ years \\
\hline Niacin $(\mathrm{mg})$ & 30 & 35 \\
\hline Vitamin B6 $(\mathrm{mg})$ & 80 & 100 \\
\hline Folate $(\mu \mathrm{g})$ & 800 & 1000 \\
\hline Choline $(\mathrm{AI}, \mathrm{mg})$ & 3000 & 3500 \\
\hline Vitamin C $(\mathrm{mg})$ & 1800 & 2000 \\
\hline Vitamin A $(\mu \mathrm{g})$ & 2800 & 3000 \\
\hline Vitamin D $(\mathrm{AI}, \mu \mathrm{g})^{2}$ & 100 & 100 \\
\hline Vitamin E $(\mathrm{mg})$ & 800 & 1000 \\
\hline Minerals & & \\
\hline Sodium $(\mathrm{AI}, \mathrm{mg})$ & 2300 & 2300 \\
\hline Chloride $(\mathrm{AI}, \mathrm{mg})$ & 3600 & 3600 \\
\hline Calcium $(\mathrm{AI}, \mathrm{mg})$ & 2500 & 2500 \\
\hline Phosphorus $(\mathrm{mg})$ & 3500 & 3500 \\
\hline Magnesium $(\mathrm{mg})$ & 350 & 350 \\
\hline Iron $(\mathrm{mg})$ & 45 & 45 \\
\hline Zinc $(\mathrm{mg})$ & 34 & 40 \\
\hline Iodine $(\mu \mathrm{g})$ & 900 & 1100 \\
\hline Selenium $(\mu \mathrm{g})$ & 400 & 400 \\
\hline Copper $(\mu \mathrm{g})$ & 8000 & 10,000 \\
\hline Manganese $(\mathrm{AI}, \mathrm{mg})$ & 9 & 11 \\
\hline Fluoride $(\mathrm{AI}, \mathrm{mg})$ & 10 & 10 \\
\hline Molybdenum $(\mu \mathrm{g})$ & 1700 & 2000 \\
\hline Boron $(\mathrm{mg})$ & 17 & 20 \\
\hline Nickel $(\mathrm{mg})$ & 1.0 & 1.0 \\
\hline 'S $\mathrm{Gr}$ & & \\
\hline
\end{tabular}

${ }^{1}$ (S. S. Gropper et al., 2009)

${ }^{2}$ (National Institutes of Health, 2014) 
APPENDIX B: NUTRIENT COMPARISON BY TREATMENT GROUP

\begin{tabular}{|c|c|c|c|}
\hline Nutrient & $\begin{array}{l}\text { RUSF } \\
\text { Amount (\%RDA) }\end{array}$ & $\begin{array}{l}\text { CSB+UNIMMAP } \\
\text { Amount (\%RDA) }\end{array}$ & $\begin{array}{l}\text { CSB-IFA }^{2} \\
\text { Amount }(\% \mathrm{RDA})\end{array}$ \\
\hline Energy (kcal) & 920 & 893 & 893 \\
\hline Protein (g) & 36 & 33 & 33 \\
\hline$\alpha$-linolenic acid (g) & $2.26(161)$ & 0 & 0 \\
\hline Linoleic acid (g) & $13.96(107)$ & 0 & 0 \\
\hline Vitamin A $(\mu \mathrm{g})$ & $2628(341)$ & $3210(417)$ & $2410(312)$ \\
\hline $\begin{array}{l}\text { Vitamin B1 } \\
\text { (Thiamin; mg) }\end{array}$ & $3.2(228)$ & $1.7(121.5)$ & $0.3(20)$ \\
\hline $\begin{array}{l}\text { Vitamin B2 } \\
\text { (Riboflavin; mg) }\end{array}$ & $3.8(270)$ & $4.7(355)$ & $3.29(235)$ \\
\hline Niacin (B3; mg) & $35(194)$ & $36.8(204)$ & $18.8(104)$ \\
\hline Vitamin B6 (mg) & $4.0(210)$ & $5.9(198)$ & $4.0(210)$ \\
\hline Vitamin $\mathrm{B}_{12}(\mu \mathrm{g})$ & $5.5(262)$ & $7.3(253)$ & $4.7(181)$ \\
\hline Folic acid $(\mu \mathrm{g})$ & $574(143)$ & $659(165)$ & $659(163)$ \\
\hline Vitamin C (mg) & $170(200)$ & $281(331)$ & $211(249)$ \\
\hline Vitamin $\mathrm{D}(\mu \mathrm{g})$ & $30(200)$ & $20.9(206)$ & $25.4(169)$ \\
\hline Vitamin E (mg) & $39.2(261)$ & $29.5(197)$ & $19.5(130)$ \\
\hline Vitamin $\mathrm{K}(\mu \mathrm{g})$ & $192(213)$ & $70.5(78)$ & $70.5(78)$ \\
\hline Iodine $(\mu \mathrm{g})$ & $300(136.4)$ & $244(170)$ & $94(43)$ \\
\hline Copper $(\mu \mathrm{g})$ & $2400(240)$ & $2000(200)$ & 0 \\
\hline Iron $(\mathrm{mg})$ & $45(170)$ & $45.3(180.7)$ & $79.2(292)$ \\
\hline Zinc (mg) & $24.6(223)$ & $26.8(243)$ & $11.8(107)$ \\
\hline Magnesium (mg) & $327(93)$ & 0 & 0 \\
\hline Calcium (mg) & $1830(183)$ & $851(85)$ & $851(85)$ \\
\hline Selenium $(\mu \mathrm{g})$ & $123(205)$ & $65(108)$ & 0 \\
\hline
\end{tabular}

${ }^{1}$ Estimates a daily portion of $235 \mathrm{~g} \mathrm{CSB}+$ per day plus UNIMMAP supplement

${ }^{2}$ Estimates a daily portion of $235 \mathrm{~g}$ CSB per day plus iron $(60 \mathrm{mg})$ and folic acid $(400 \mu \mathrm{g})$ 


\section{APPENDIX C: MICRONUTRIENT SUB-STUDY FORM}

version 4

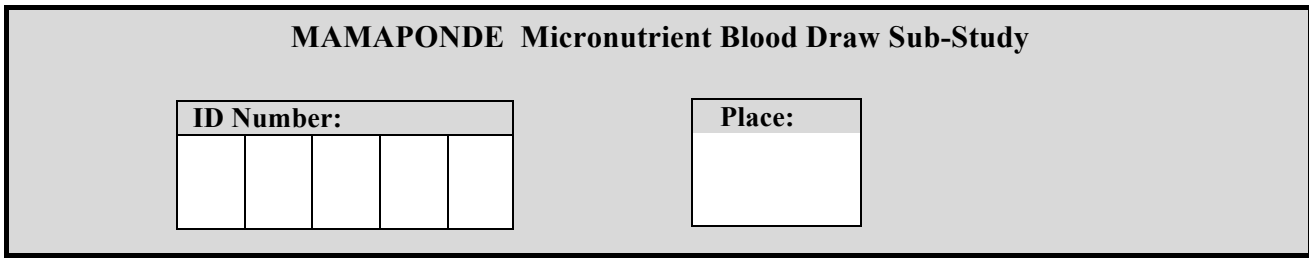

\begin{tabular}{|l|l|}
\hline DATE OF FIRST BLOOD DRAW: & DATE OF SECOND BLOOD DRAW: \\
\hline$(\mathrm{dd} / \mathrm{mm} / \mathrm{yy})$ & $\frac{(\mathrm{dd} / \mathrm{mm} / \mathrm{yy})}{}$
\end{tabular}

WEEKS GESTATION AT ENROLLMENT (enrollment criteria $\leq 26$ weeks):

WEEKS GESTATION AT $2^{\mathrm{ND}}$ VISIT:

\begin{tabular}{|l|l|}
\hline \multicolumn{1}{|c|}{ PLACE BARCODE $1^{\text {ST }}$ BLOOD DRAW } & \\
\hline & \\
\hline PLACE BARCODE ${ }^{\text {ND }}$ BLOOD DRAW \\
\hline Write number here: & Write number here: \\
\hline
\end{tabular}

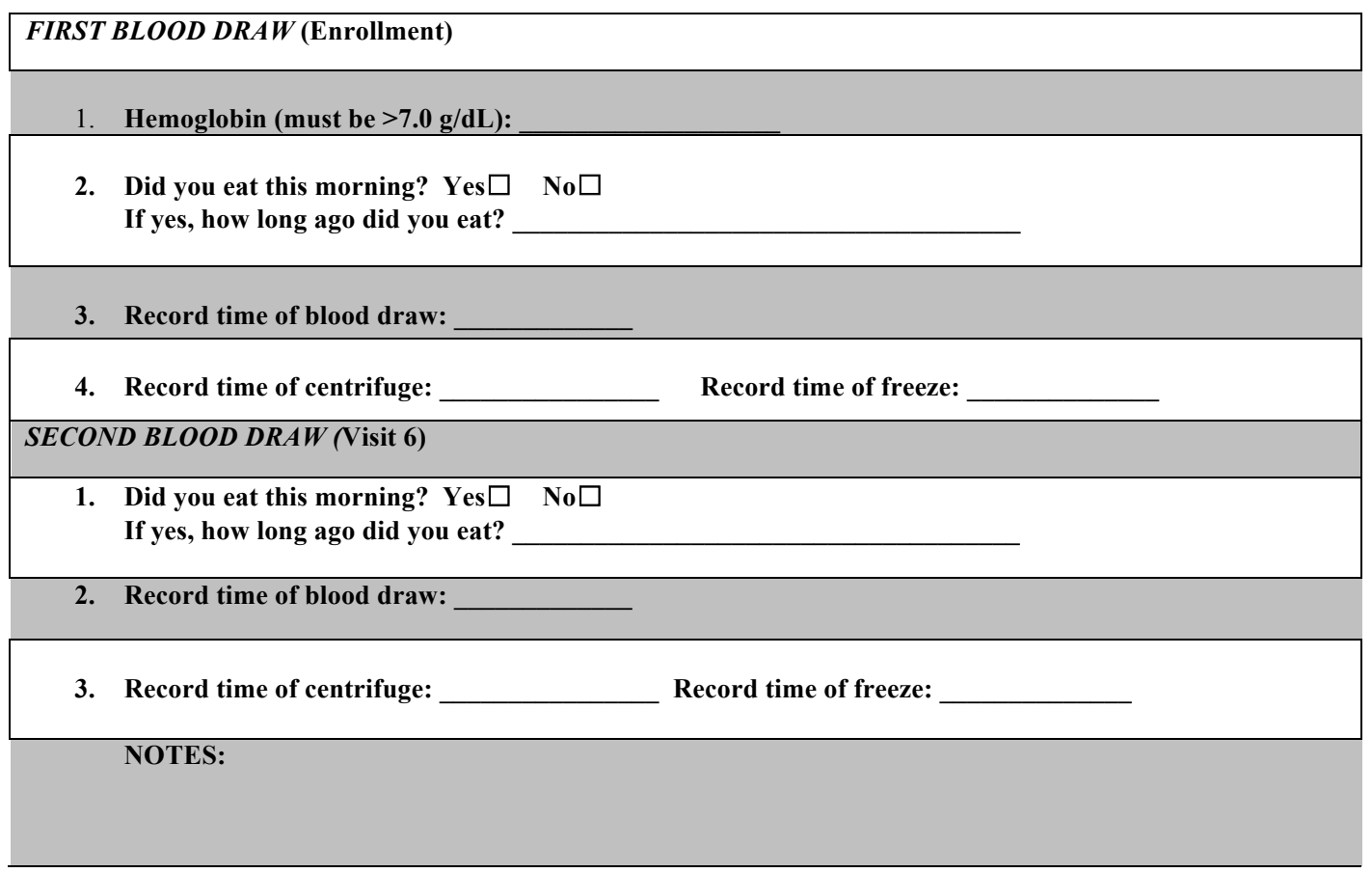




\section{$*$ BLUE tube FIRST $\rightarrow$ Half full $\rightarrow$ Invert 8-10x AT CLINIC: \\ *TIGER TOP tube SECOND $\rightarrow$ All the way full $\rightarrow$ Invert $5 x$}

1. Nurse will get informed consent from the woman.

2. Nurse will make sure the woman is 26 weeks gestation or lower. We want to make sure she doesn't deliver before the second blood draw.

3. Make sure hemoglobin has been done and that it is above $7.0 \mathrm{~g} / \mathrm{dL}$.

4. Nurse will ask questions on blood draw form (about food).

5. Nurse will wash hands and put on new gloves.

6. Volunteer will prepare cup of Sobo and 4 cookies.

7. Volunteer will tear off the next 7 identical barcodes from the roll of barcodes. Make sure they all have the same number.

*NOTE*: We have 2 rolls of barcodes. One roll has numbers starting with "10001". These are for the first blood draw. The second roll has numbers starting with "20001". These are for the second blood draw. The end numbers should be the same for each woman.

○ Example: The first woman will have "10001" for her first blood draw and "20001" for her second blood draw.

8. Volunteer will place one barcode label on the woman's paper form.

9. Do not put the other barcodes on the tubes until after the blood draw is completed and acceptable.

10. Identify a vein to be used for blood draw.

*NOTE: Veins will be more prominent if she forms a fist with her hand

11. Volunteer will get out one tiger top tube, one dark blue tube, one safety holder, a needle, cotton, and tape.

12. Volunteer will record time of blood draw on the paper form.

13. Select a 21 gauge needle (green cap) if the woman has normal sized veins.

14. Select a 22 gauge needle (black cap) if the woman has small sized veins.

*NOTE: 21 gauge is bigger than 22 gauge. Try to use the 21 gauge needle if possible because there is usually better blood flow.

15. Nurse will clean the arm around the vein site with cotton and mineral spirits. Let it dry before taking blood.

16. Tie a tourniquet around woman's arm above a few inches above her elbow.

17. Unscrew the clear top from the needle.

18. Insert grey rubber end pointed down into the orange part of the safety holder and screw in place tightly.

19. Gently place the dark blue tube onto grey rubber side of needle, but do not puncture it yet! Make sure the label is facing down so you can see the insides of the tube. 
20. Pull off green (or black) cap from needle, carefully.

21. Hold the arm and place your thumb below the blood draw site.

22. Insert the needle into the vein with the orange safety cap facing up towards you AND with the "bevel" facing up. The bevel is the larger opening of the needle. You can spin the orange safety cap around until both are in the upright position.

23. After puncturing the skin, make sure you have a steady grip with one hand. With the other hand, push the dark blue tube up all the way so the needle punctures it until blood starts flowing in.

*NOTE: ALWAYS fill up the dark blue tube FIRST, because the tiger top tube has special ingredients in it that will contaminate the dark blue tube.

24. Fill up the dark blue tube only half way (about $3 \mathrm{~mL}$ ).

25. Remove it from the safety holder and place it upright in the rack.

26. NEVER remove the rubber top of tube because the vacuum pressure will be released.

27. Pierce the tiger top tube into the safety holder side and fill it all the way until you get to the top line on the tube.

28. Remove the tiger top tube and place it upright in a rack.

29. Take off tourniquet.

30. Remove the needle from the arm and apply firm pressure with a cotton ball to the puncture site with one hand.

31 . With the other hand, carefully push the safety holder onto the needle using a table or hard surface, until you hear it click.

32. Put the entire needle and holder into a biohazard/sharps container.

33. Place a piece of tape over the cotton ball on the arm.

34. Place one barcode label on the tiger top tube and one label on the dark blue tube. Make sure that the numbers on the tubes match the number on her paper form.

35. Place the remaining barcodes into a Ziplock bag in the cooler to be taken to the lab.

36. Invert the dark blue tube 8 to 10 times.

37. Cover the dark blue tube with foil and place it into the small cooler with ice packs.

38. Invert the tiger top tube up and down 5 times.

39. Let the tiger top tube sit out for up to 30 minutes to allow the blood to clot. After 30 minutes, cover the tiger top tube with foil and place it into the cooler with ice packs for transport to the laboratory at the College of Medicine.

40. Write gift (plates\&Soap for first blood draw or chitenje for second blood draw) on woman's passbook!

41. Write "BLOOD" at Visit 6 on Clinic Card

\section{AT THE LAB:}

1. Put on safety equipment. 
2. Record time of centrifuge.

3. Centrifuge tubes at $3500 \mathrm{rpm}$ for 10 minutes, keeping temperature below 25 degrees $\mathrm{C}$.

4. If there are an odd number of tubes for any reason, fill up a tiger top tube or dark blue tube with water to create a balance tube to go across in the centrifuge.

5. Take out the remaining barcodes from the cooler.

6. While it's centrifuging, hand write the Study ID number and the barcode label number on a separate sheet of paper. This will be transferred to an Excel file later.

7. Only do one woman's blood at a time (one tiger top tube and one dark blue tube) to avoid mix-ups.

8. Find the barcode labels that match the labels on the tiger top and dark blue tube that you are about to use.

9. Put one label on a clear transport tube, one label on an amber tube, and one label on another amber tube.

10. With a Sharpie, write " 1 " on one amber tube and " 2 " on the other amber tube. Write it above the barcode label below the cap. "Amber 1" will be the tube to be analyzed at Central Coast Pathology. "Amber 2" will be the tube to be sent to Bakersfield lab.

11. In a rack, place the tubes in the following order from left to right: Tiger top tube, dark blue tube, amber 1 , amber 2 , clear tube.

12. Place a pipette tip on the pipette.

13. From the tiger top tube, pipette $2 \mathrm{~mL}$ of serum into Amber 1 and $2 \mathrm{~mL}$ of serum into Amber 2.

14. Discard the pipette tip into the biohazard container.

15. Place a new pipette tip on the pipette.

16. From the dark blue tube, about $2-3 \mathrm{~mL}$ of serum will be transferred into a trace mineral free transport tube.

17. Scan with the barcode scanner to ensure each woman has 5 tubes (1 tiger top, 1 dark blue, 2 amber, 1 clear transport tube) matched with the barcode label on her paper form.

18. Store the amber tubes and clear tubes in racks in -70 degree Celsius freezer at the College of Medicine until they are transported back to Central Coast Pathology for analysis.

19. Dispose of the SST and dark blue tubes properly into a biohazard container. 


\section{APPENDIX E: MAMAPONDE MICRONUTRIENT BLOOD DRAW INFORMED}

\section{CONSENT}

\section{Informed Consent: Vitamin and Mineral Study}

\section{Assessment of micronutrient status in pregnant Malawian women before and after treatment for moderate malnutrition: a randomized controlled trial}

You are invited to participate in a research study conducted by Cambria Glosz, Dr. Peggy

Papathakis, Dr. Manary and colleagues.

The study is looking at levels of vitamins and minerals in your body. Our study is trying to help you recover from malnutrition.

1. The purpose of this study is to see if the levels of vitamins and minerals in your body change with the food supplement, and which one of the three supplements helps pregnant women the best in terms of vitamins and minerals.

2. You will have your blood drawn to assess your vitamin and mineral levels two times: The first time will be today when you enroll in the study, and once more about 10-12 weeks later after you have recovered from malnutrition or before delivery if you have not yet recovered. Each time, we will draw approximately $8-10 \mathrm{~mL}$ or about 2 teaspoons of blood from your arm. It is possible that you might have some pain or bruising at the site where the blood was drawn. To minimize these risks, a trained Malawian study nurse will be drawing your blood, using Universal Precautions.

3. You will receive plates and soap the first time you have your blood drawn and you will receive a chitenje the second time you have your blood drawn. There is no cost to you to participate.

4. If you agree you volunteer to participate, you may withdraw consent and discontinue your participation at any time without penalty. You may also choose not to participate in specific procedures or answer specific questions if you would prefer not to.

5. All reasonable measures to protect the confidentiality of your medical records and your identity will be taken. There is a possibility that your medical record, including identifying information, may be inspected and photocopied by government or University officials or members of the Human Studies Committee.

6. If you have any questions or concerns regarding this study, if any problems arise, or there any feelings of pressure to participate, you may call the Principal Investigator Cambria Glosz at (858) 722-8231, Dr. Peggy Papathakis at (805) 756-7205, or Dr. Mark Manary at Queen Elizabeth Central Hospital in Blantyre, Malawi at 0999-921-656. If you have questions or concerns regarding the manner in which the study is conducted, you may contact Dr. Steve Davis, Chair of the Cal Poly Human Subjects Committee, at (805) 756-2754, sdavis@calpoly.edu, or Dr. Dean Wendt, Interim Dean of Research, at (805) 756-1508,dwendt@calpoly.edu.You may also ask questions or state concerns regarding your rights as a research subject to Dr. Kalanda, the COMREC chair at the University of Malawi, College of Medicine at 0999460737 locally if you have any questions or concerns about this study. 
7. You will be informed of any significant new findings developed during the course of participation in this research that may have a bearing on your willingness to continue in the study. This vitamin and mineral study is not intended to diagnose or treat any medical problems not specifically stated in the purpose of the research. If you would like to be informed of any results from this study, please contact the researchers using the contact information above.

I have read this consent form and have been given the opportunity to ask questions. I will also be given a copy of this consent form for my records. I hereby give my consent to participate in the research described above.

I voluntarily agree to take part:

Name of Volunteer Participant (signed) Date

Name of Volunteer Participant (printed)

Signature of Researcher Date

Thumbprint:

\begin{tabular}{|l|l|l|l|}
\hline DATE & STUDY ID & & $\begin{array}{l}\text { SIGNATURE, } \\
\text { INITIALS, OR } \\
\text { THUMPRINT }\end{array}$ \\
\hline & & & \\
\hline & & & \\
\hline & & & \\
\hline & & & \\
\hline & & & \\
\hline & & & \\
\hline & & & \\
\hline & & & \\
\hline & & & \\
\hline
\end{tabular}

